\title{
Central Nervous System Cancers, Version 3.2020
}

Louis Burt Nabors, MD ${ }^{1, *}$; Jana Portnow, MD2,*; Manmeet Ahluwalia, MD; Joachim Baehring, MD4; Henry Brem, MD5; Steven Brem, MD ${ }^{6}$; Nicholas Butowski, MD; Jian L. Campian, MD, PhD ${ }^{8}$; Stephen W. Clark, MD, PhD; Andrew J. Fabiano, MD ${ }^{10}$; Peter Forsyth, MD ${ }^{11}$; Jona Hattangadi-Gluth, MD ${ }^{12}$; Matthias Holdhoff, MD, PhD ${ }^{5, *}$; Craig Horbinski, MD, PhD ${ }^{13, *}$; Larry Junck, MD ${ }^{14}$; Thomas Kaley, MD ${ }^{15, *}$; Priya Kumthekar, MD ${ }^{13, \star}$; Jay S. Loeffler, MD ${ }^{16}$; Maciej M. Mrugala, MD, PhD, MPH' ${ }^{17, *}$; Seema Nagpal, MD ${ }^{18, *}$; Manjari Pandey, MD ${ }^{19}$; lan Parney, MD, PhD ${ }^{17}$; Katherine Peters, MD, PhD ${ }^{20}$; Vinay K. Puduvalli, MD²1,*; lan Robins, MD, PhD 22 ; Jason Rockhill, MD, PhD ${ }^{23}$; Chad Rusthoven, MD ${ }^{24, *}$; Nicole Shonka, MD ${ }^{25}$; Dennis C. Shrieve, MD, PhD ${ }^{26}$; Lode J. Swinnen, MB, ChB ${ }^{5, *}$; Stephanie Weiss, MD ${ }^{27}$; Patrick Yung Wen, MD ${ }^{28, *}$; Nicole E. Willmarth, PhD ${ }^{29}$; Mary Anne Bergman ${ }^{30}$; and Susan D. Darlow, $\mathrm{PhD}^{30}$

\section{ABSTRACT}

The NCCN Guidelines for Central Nervous System (CNS) Cancers focus on management of adult CNS cancers ranging from noninvasive and surgically curable pilocytic astrocytomas to metastatic brain disease. The involvement of an interdisciplinary team, including neurosurgeons, radiation therapists, oncologists, neurologists, and neuroradiologists, is a key factor in the appropriate management of CNS cancers. Integrated histopathologic and molecular characterization of brain tumors such as gliomas should be standard practice. This article describes NCCN Guidelines recommendations for WHO grade I, II, III, and IV gliomas. Treatment of brain metastases, the most common intracranial tumors in adults, is also described.

J Natl Compr Canc Netw 2020;18(11):1537-1570 doi: $10.6004 /$ jnccn.2020.0052

${ }^{1} \mathrm{O}$ 'Neal Comprehensive Cancer Center at UAB; ${ }^{2} \mathrm{C}$ ity of Hope National Medical Center; ${ }^{3}$ Case Comprehensive Cancer Center/University Hospitals Seidman Cancer Center and Cleveland Clinic Taussig Cancer Institute; ${ }^{4}$ Yale Cancer Center/Smilow Cancer Hospital; ${ }^{5}$ The Sidney Kimmel Comprehensive Cancer Center at Johns Hopkins; ${ }^{6}$ Abramson Cancer Center at the University of Pennsylvania; ${ }^{7}$ UCSF Helen Diller Family Comprehensive Cancer Center; ${ }^{8}$ Siteman Cancer Center at Barnes-Jewish Hospital and Washington University School of Medicine; ${ }^{9}$ Vanderbilt-Ingram Cancer Center; ${ }^{10}$ Roswell Park Comprehensive Cancer Center; ${ }^{11}$ Moffitt Cancer Center; ${ }^{12}$ UC San Diego Moores Cancer Center; ${ }^{13}$ Robert H. Lurie Comprehensive Cancer Center of Northwestern University; ${ }^{14}$ University of Michigan Rogel Cancer Center;

${ }^{15}$ Memorial Sloan Kettering Cancer Center; ${ }^{16}$ Massachusetts General Hospital Cancer Center; ${ }^{17}$ Mayo Clinic Cancer Center; ${ }^{18}$ Stanford Cancer Institute;

${ }^{19} \mathrm{~S}$ t. Jude Children's Research Hospital/The University of Tennessee Health Science Center; ${ }^{20}$ Duke Cancer Institute; ${ }^{21}$ The Ohio State University

Comprehensive Cancer Center - James Cancer Hospital and Solove Research Institute; ${ }^{22}$ University of Wisconsin Carbone Cancer Center; ${ }^{23}$ Fred Hutchinson Cancer Research Center/Seattle Cancer Care Alliance; ${ }^{24}$ University of Colorado Cancer Center; ${ }^{25}$ Fred and Pamela Buffet Cancer Center; ${ }^{26}$ Huntsman Cancer Institute at the University of Utah; ${ }^{27}$ Fox Chase Cancer Center; ${ }^{28}$ Dana-Farber Cancer Institute; ${ }^{29}$ American Brain Tumor Association; and ${ }^{30}$ National

Comprehensive Cancer Network

*Discussion Writing Committee Member.

\section{NCCN CATEGORIES OF EVIDENCE AND CONSENSUS}

Category 1: Based upon high-level evidence, there is uniform NCCN consensus that the intervention is appropriate.

Category 2A: Based upon lower-level evidence, there is uniform NCCN consensus that the intervention is appropriate.

Category 2B: Based upon lower-level evidence, there is NCCN consensus that the intervention is appropriate.

Category 3: Based upon any level of evidence, there is major NCCN disagreement that the intervention is appropriate.

All recommendations are category $2 \mathrm{~A}$ unless otherwise noted.

Clinical trials: NCCN believes that the best management of any patient with cancer is in a clinical trial. Participation in clinical trials is especially encouraged.

\section{PLEASE NOTE}

The NCCN Clinical Practice Guidelines in Oncology (NCCN Guidelines ${ }^{\circledR}$ ) are a statement of evidence and consensus of the authors regarding their views of currently accepted approaches to treatment. Any clinician seeking to apply or consult the NCCN Guidelines is expected to use independent medical judgment in the context of individual clinical circumstances to determine any patient's care or treatment. The National Comprehensive Cancer Network ${ }^{\circledR}\left(\mathrm{NCCN}^{\circledR}\right)$ makes no representations or warranties of any kind regarding their content, use, or application and disclaims any responsibility for their application or use in any way.

The complete NCCN Guidelines for Central Nervous System Cancers are not printed in this issue of JNCCN but can be accessed online at NCCN.org.

(C) National Comprehensive Cancer Network, Inc. 2020. All rights reserved. The NCCN Guidelines and the illustrations herein may not be reproduced in any form without the express written permission of NCCN.

\section{Disclosures for the NCCN Central Nervous System Cancers Panel}

At the beginning of each NCCN Guidelines Panel meeting, panel members review all potential conflicts of interest. NCCN, in keeping with its commitment to public transparency, publishes these disclosures for panel members, staff, and NCCN itself.

Individual disclosures for the NCCN Central Nervous System Cancers Panel members can be found on page 1570. (The most recent version of these guidelines and accompanying disclosures are available at NCCN.org.)

The complete and most recent version of these guidelines is available free of charge at NCCN.org. 


\section{Adult Low-Grade (WHO Grade I or II) Glioma/Pilocytic and Infiltrative Supratentorial Astrocytoma/Oligodendroglioma}

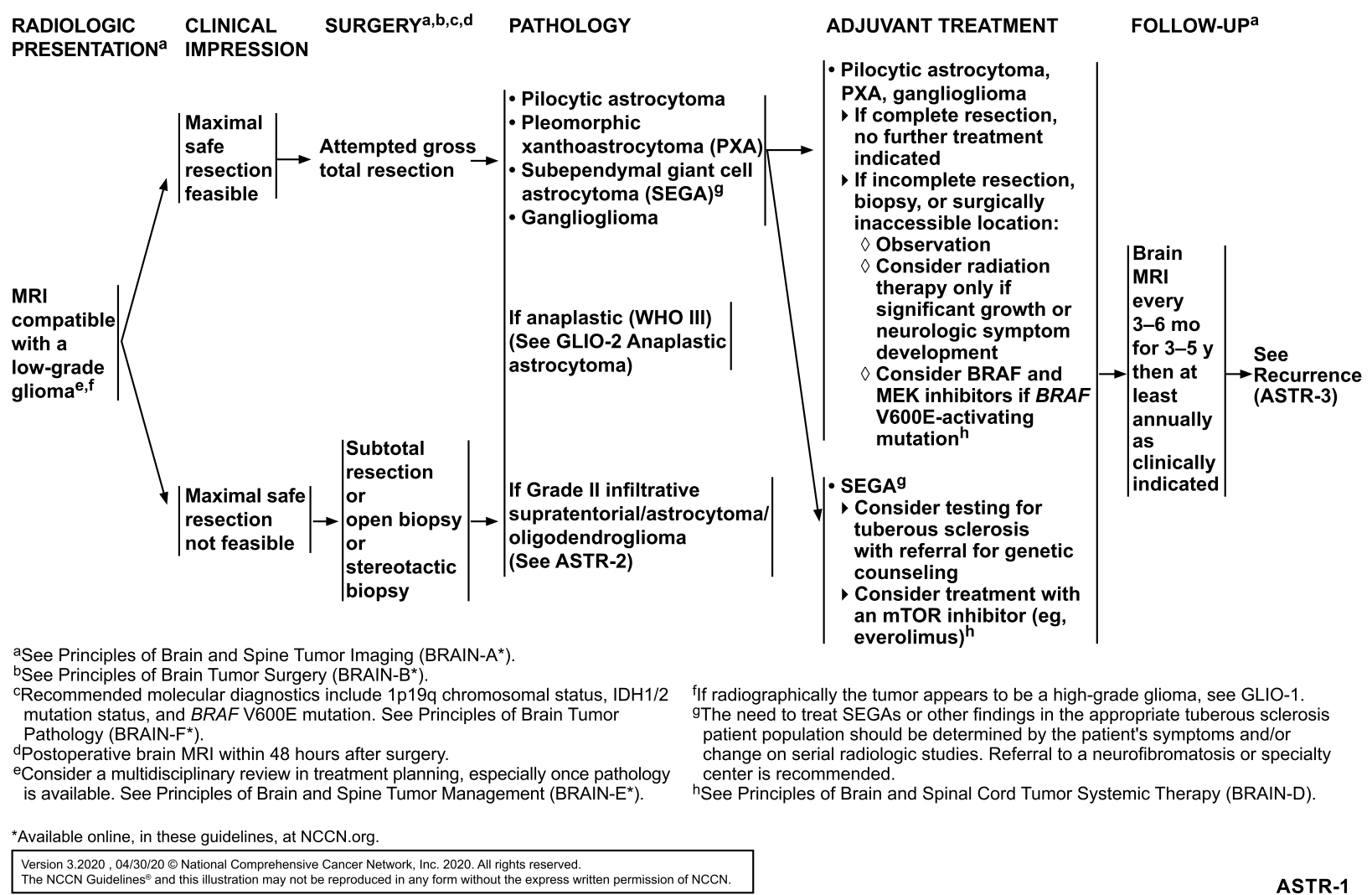

\section{Overview}

Primary brain tumors are a heterogeneous group of neoplasms with varied outcomes and management strategies. Primary malignant brain tumors range from pilocytic astrocytomas, which are very uncommon, noninvasive, and surgically curable, to glioblastoma, the most common malignant brain tumor in adults, which is highly invasive and virtually incurable. Likewise, patients with metastatic brain disease may have rapidly progressive systemic disease or no systemic cancer at all. These patients may have one or many brain metastases, and they may have a malignancy that is highly responsive or, alternatively, highly resistant to radiation therapy (RT) or chemotherapy. Because of this marked heterogeneity, the prognostic features and treatment options for primary and metastatic brain tumors must be carefully reviewed on an individual basis and sensitively communicated to each patient. In addition, central nervous system (CNS) tumors are associated with a range of symptoms such as seizures, fatigue, impaired short term memory, changes in cognition or behavior (particularly anxiety and depression), impaired mobility, impairment of speech and comprehension, and visual impairment, as well as complications such as intracerebral edema, infections, endocrinopathies, and venous thromboembolism that can seriously impact patients' quality of life.

\section{Gliomas}

The NCCN Guidelines for CNS Cancers include recommendations for management of the following gliomas ${ }^{1}$ :

- Grade I: pilocytic astrocytoma, pleomorphic xanthoastrocytoma, ganglioglioma, subependymal giant cell astrocytoma

- Grade II: diffuse astrocytoma and oligodendroglioma

- Grade III: anaplastic astrocytoma and oligodendroglioma

- Grade IV: glioblastoma

\section{Molecular Profiling for Gliomas}

Integrated histopathologic and molecular characterization of gliomas should be standard practice. Molecular/ genetic characterization complements standard histologic analysis, providing additional diagnostic and prognostic 


\section{Adult Low-Grade (WHO Grade I or II) Glioma/Pilocytic and Infiltrative Supratentorial Astrocytoma/Oligodendroglioma} PRESENTATIONc,e,f

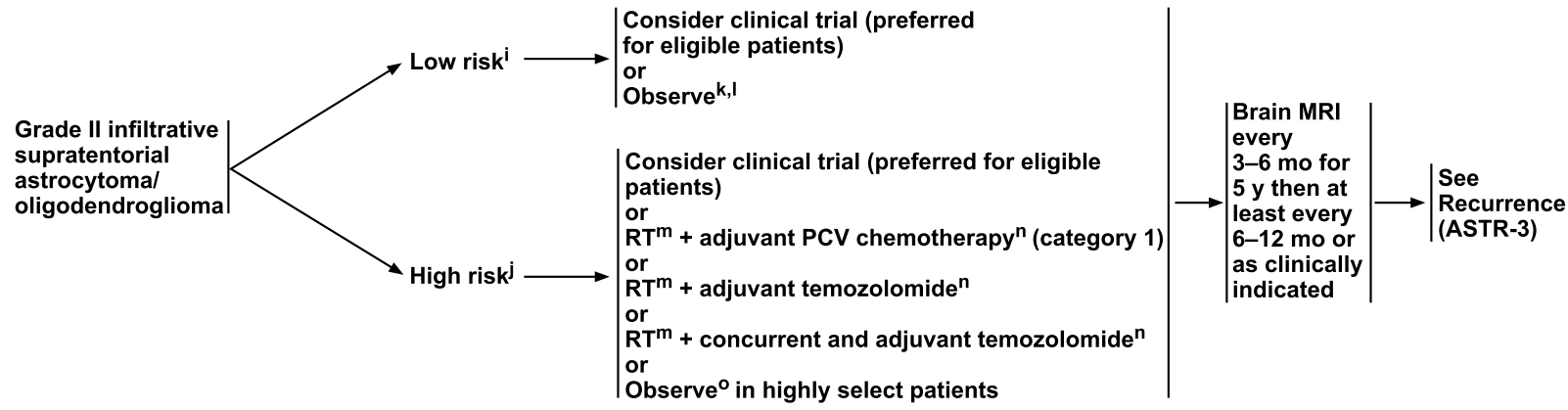

\footnotetext{
cRecommended molecular diagnostics include 1p19q chromosomal status, IDH1/2 mutation status, and BRAF V600E mutation. See Principles of Brain Tumor Pathology (BRAIN-F*).

e Consider a multidisciplinary review in treatment planning, especially once

pathology is available. See Principles of Brain and Spine Tumor Management pathology is

If radiographically the tumor appears to be a high-grade glioma, see GLIO-1.

'Low-risk features: $\leq 40$ y and gross total resection (GTR).

jHigh-risk features: $>40$ y or subtotal resection (STR) or open or stereotactic biopsy.

Other high-risk factors that are sometimes taken into consideration are tumor size,

neurologic deficits, and presence of sequencing verified IDH wild type.

kRegular follow-up is essential for patients receiving observation alone after resection.

*Available online, in these guidelines, at NCCN.org

In the event that other risk factors are considered and treatment is warranted, treat as high risk. There may also be rare circumstances in which treating a patient with fractionated external beam RT alone (category $2 \mathrm{~B}$ ) or chemotherapy alone (category 2B) may be considered. See Principles of Brain and Spinal Cord Tumor Radiation (BRAIN-C*) or Principles of Brain and Spinal Cord Tumor Systemic Therapy (BRAIN-D).

mFor low-grade gliomas, See Principles of Brain and Spinal Cord Tumor Radiation Therapy (BRAIN-C*).

nsee Principles of Brain and Spinal Cord Tumor Systemic Therapy (BRAIN-D).

oThe results of RTOG 9802 showed that there was a significant improvement in 'The results of RTOG 9802 showed that there was a significant improvement in
median overall survival in high-risk low-grade glioma patients treated with RT followed by PCV $\times 6$ cycles compared with RT alone after a tissue diagnosis was made. However, this important study did not address whether all of these patients should be treated right away. Observation after diagnosis may be a reasonable option for a high-risk low-grade glioma patient who is neurologically asymptomatic or stable. Close monitoring with brain MRIs is important.

information that improves diagnostic accuracy and aids in treatment selection.

\section{Updated Classification of Gliomas Based on Histology and Molecular Features}

In 2016, the WHO classification for grade II-III gliomas was revised as follows: (1) oligodendrogliomas are now defined as tumors that have $1 \mathrm{p} 19 \mathrm{q}$ codeletion and IDH mutation (unless molecular data are not available and cannot be obtained, in which case designation can be based on histology with appropriate caveats); (2) anaplastic gliomas were further subdivided according to IDH mutation status; (3) oligoastrocytoma is no longer a valid designation unless molecular data (1p19q codeletion and $I D H$ mutation status) are not available and cannot be obtained. ${ }^{1}$ Such tumors should be described as "oligoastrocytoma, not otherwise specified (NOS)" to indicate that the characterization of the tumor is incomplete. Very rare cases of concurrent, spatially distinct oligodendroglioma (1p19q codeleted) and astrocytoma (1p19q intact) components in the same tumor may also be labeled oligoastrocytoma. ${ }^{1}$
It is important to note that correlations between the molecularly defined 2016 WHO categories and the histology-based 2007 WHO categories are limited and vary across studies. ${ }^{2-5}$ Thus, the change from 2007 WHO to 2016 WHO reclassified a significant proportion of gliomas.

Multiple independent studies on gliomas have conducted genome-wide analyses evaluating an array of molecular features (eg, DNA copy number, DNA methylation, protein expression) in large populations of patients with grade II-IV tumors. ${ }^{4,6,7}$ Unsupervised clustering analyses, an unbiased method for identifying molecularly similar tumors, have been used to identify subgroups of gliomas with distinct molecular profiles., ${ }^{4,6}$ Remarkably, further analysis has shown that these molecular subgroups could be distinguished based on only a handful of molecular features, including mutation of IDH1/2 and 1p19q codeletion, biomarkers independently verified by many studies as hallmarks for distinguishing molecular subgroups in grade II-III gliomas. ${ }^{2-5,7-13}$ Using these markers alone, the majority of grade II-III tumors can be divided into 3 molecular subtypes: (1) mutation of either 


\section{Adult Low-Grade (WHO Grade I or II) Glioma/Pilocytic and Infiltrative Supratentorial Astrocytoma/Oligodendroglioma RECURRENCE ${ }^{\mathrm{p}}$}

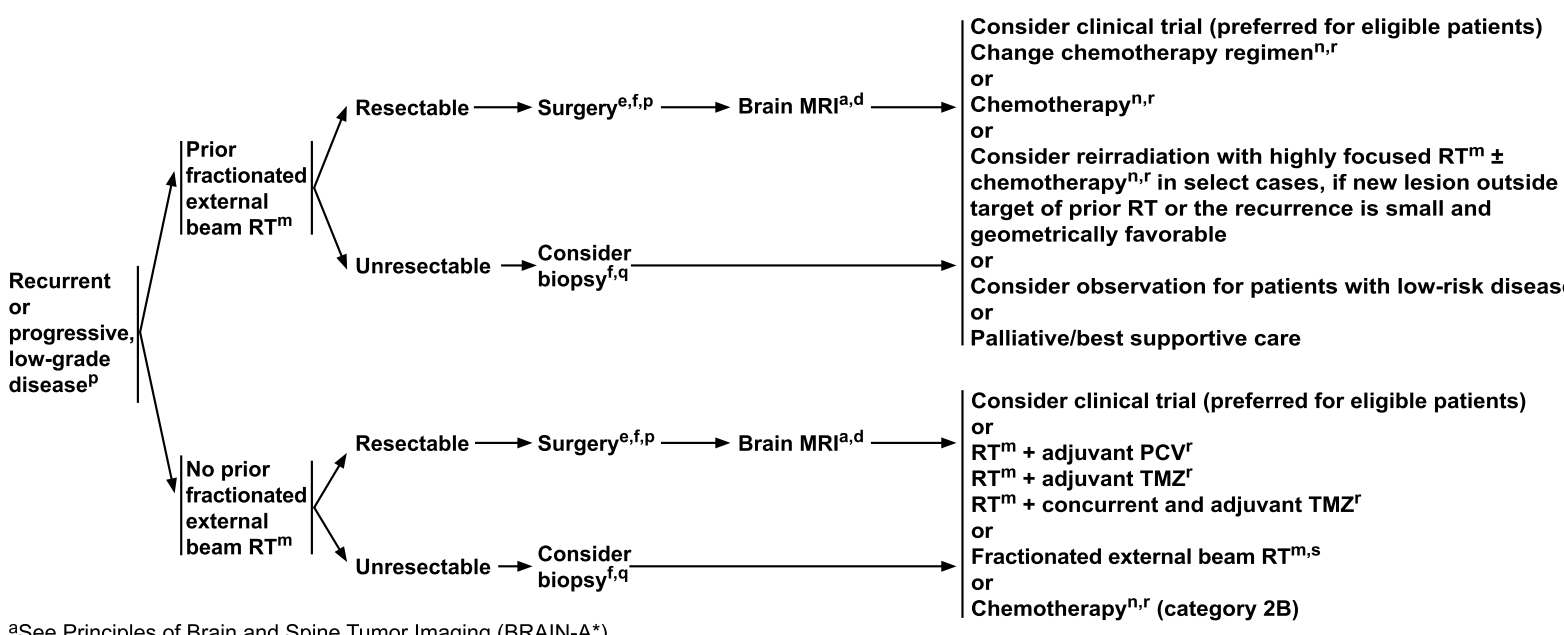

asee Principles of Brain and Spine Tumor Imaging (BRAIN-A*).

dPostoperative brain MRI within 48 hours after surgery.

e Consider a multidisciplinary review in treatment planning, especially once pathology is available. See Principles of Brain and Spine Tumor Management (BRAIN-E*).

fIf radiographically the tumor appears to be a high-grade glioma, see GLIO-1.

mFor low-grade gliomas, See Principles of Brain and Spinal Cord Tumor Radiation Therapy (BRAIN-C*).

nsee Principles of Brain and Spinal Cord Tumor Systemic Therapy (BRAIN-D).

PIf GTR is achieved in a patient with low-risk disease, consider further observation.

qRecurrence on neuroimaging can be confounded by treatment effects. To confirm tumor recurrence and assess for possible transformation of tumor to higher grade

strongly consider tumor tissue sampling (biopsy at minimum) if there is a high index of suspicion of recurrence. Sixty percent or more of astrocytomas and $40 \%-50 \%$ of

oligodendrogliomas will eventually undergo transformation to a higher grade. For treatment of patients with transformation to high-grade disease, see GLIO-1.

rBrain MRI every 2-3 months while on treatment, then every 6 months indefinitely, to assess disease recurrence/progression during treatment with chemotherapy.

(See BRAIN-A*).

SRT alone is not encouraged, but may be appropriate for select cases (eg, poor performance status).

*Available online, in these guidelines, at NCCN.org

Version 3.2020, 04/30/20 ๑ National Comprehensive Cancer Network, Inc. 2020. All rights reserved.

The NCCN Guidelines ${ }^{\varpi}$ and this illustration may not be reproduced in any form without the express written permission of NCCN

IDH1 or IDH2 (IDH-mut) with $1 \mathrm{p} 19 \mathrm{q}$ codeletion $(1 \mathrm{p} 19 \mathrm{q}$ codel); (2) $I D H$-mut with no $1 \mathrm{p} 19 \mathrm{q}$ codeletion or with isolated deletion of $1 \mathrm{p}$ or 19q; and (3) no mutation of $I D H 1$ or $I D H 2$ (IDH wild type; $I D H$-wt). ${ }^{4}$ Multiple studies have shown that the $1 \mathrm{p} 19 \mathrm{q}$ codeletion is strongly associated with $I D H$ mutations, such that true whole-arm $1 \mathrm{p} 19 \mathrm{q}$ codeletion in $I D H$-wt tumors is extremely rare..$^{2,3,10,14,15}$ In a tumor that is equivocal, the presence of an $I D H \mathrm{mu}-$ tation indicates at least a grade II diffusely infiltrative glioma. ${ }^{16}$ Grade I noninfiltrative gliomas do not have $I D H$ mutations. ${ }^{16}$

Other mutations commonly detected in gliomas can have diagnostic and prognostic value, such as those involving the histone chaperone protein, ATRX, which are most often found in grade II-III gliomas and secondary glioblastomas. ${ }^{17,18}$ ATRX mutation is robustly associated with $I D H$ mutations, and this combination is strongly suggestive of astrocytoma. ${ }^{19}$ In contrast, ATRX mutation is nearly always mutually exclusive with $1 p 19 q$ codeletion. Therefore, a glioma that has loss of normal ATRX immunostaining is unlikely to be an oligodendroglioma. Mutations in the promoter region of the telomerase reverse transcription (TERT) gene occur frequently in glioblastomas and oligodendrogliomas. ${ }^{20,21}$ TERT promoter mutations in gliomas are associated with $1 \mathrm{p} 19 \mathrm{q}$ codeletion and $I D H$ mutations in oligodendrogliomas. ${ }^{22}$ Interestingly, they are also highly characteristic of $I D H$-wt, ATRX wild-type glioblastomas, especially those that contain amplification of epidermal growth factor receptor (EGFR). ${ }^{20,21} \mathrm{H} 3 \mathrm{~K} 27 \mathrm{M}$ mutations in the histone-encoding $H 3 F 3 A$ gene are mostly found in diffuse midline gliomas in both children and adults. ${ }^{23}$ Patients with these $H 3 K 27 M$ mutated gliomas tend to have a very poor prognosis regardless of histologic appearance, so they are classified as WHO grade IV. ${ }^{22,23}$

Analyses of large databases have also suggested a number of other molecular markers as being potential characteristic/prognostic features of specific subgroups. ${ }^{3,5,7,10,14,19}$ Molecular features suggested as markers for subtyping grade II-III gliomas include mutations in NOTCH1, CIC, FUBP1; mutation in TP53 and/or overexpression of aberrant TP53; PTEN loss or promoter methylation; amplification of EGFR; and chromosome 7 gain, chromosome 10 loss. ${ }^{2,4,5,11,22}$ Due to 
Anaplastic Gliomas ${ }^{2} /$ Glioblastoma

$\begin{array}{llll}\text { RADIOLOGIC } & \text { CLINICAL } & \text { SURGERYc } & \text { PATHOLOGYd } \\ \text { PRESENTATION } & \text { IMPRESSION } & \text { SU }\end{array}$

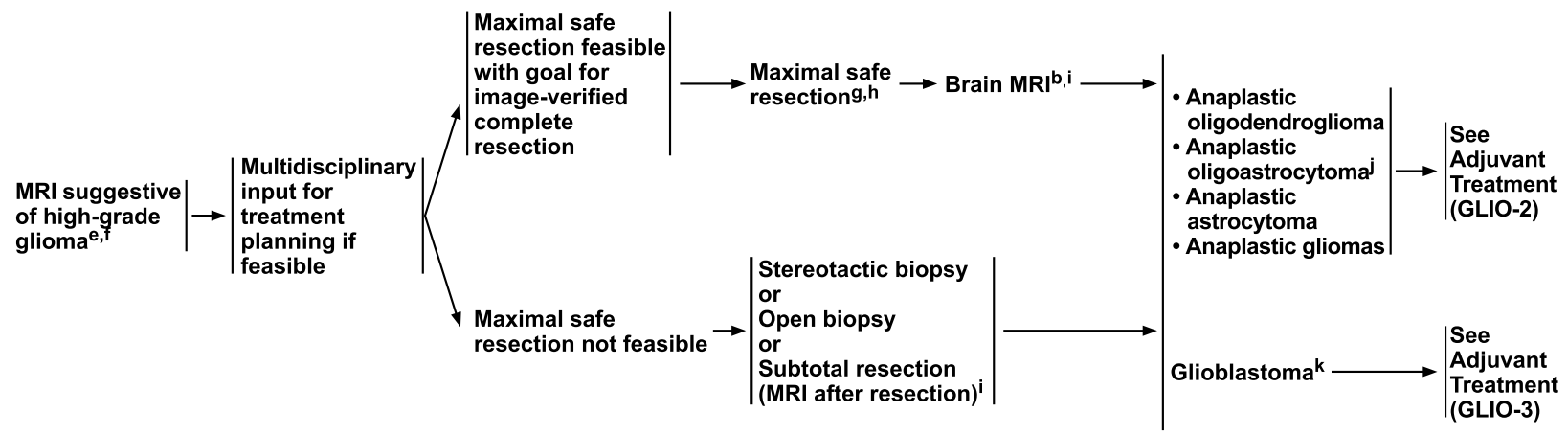

aThis pathway includes the classification of mixed anaplastic oligoastrocytoma (AOA), anaplastic astrocytoma (AA), anaplastic oligodendroglioma (AO), and other rare anaplastic gliomas.

bSee Principles of Brain and Spine Tumor Imaging (BRAIN-A*).

cSee Principles of Brain Tumor Surgery (BRAIN-B*).

dSee Principles of Brain Tumor Pathology (BRAIN-F*).

eBiopsy prior to administration of steroids if MRI compatible with CNS lymphoma.

${ }^{f}$ Consider a multidisciplinary review in treatment planning, especially once pathology

is available. See Principles of Brain and Spine Tumor Management (BRAIN-E*).

gIf frozen section diagnosis supports high-grade glioma.

hConsider carmustine (BCNU) wafer implant during maximal safe resection (category $2 \mathrm{~B}$ ).

Treatment with carmustine wafer may impact enrollment in adjuvant clinical trials.

iPostoperative brain MRI within 48 hours after surgery.

jThe 2016 WHO Classification of Tumors of the CNS has deleted

oligoastrocytoma as a category, although "anaplastic oligoastrocytoma, NOS" may continue to be used for 1) patients with mixed histology and no available molecular data (ie, no tissue available for analysis) for determining whether to classify as oligodendroglioma versus astrocytoma; or 2) rare instances in which the tumor has regions with histologic features of oligoastrocytoma with $1 \mathrm{p} 19 \mathrm{q}$-codeletion, and distinct regions with histologic features of astrocytoma without $1 \mathrm{p} 19 \mathrm{q}$-codeletion.

*Available online, in these guidelines, at NCCN.org

without $1 \mathrm{p} 19 \mathrm{q}-$-codeletion.
$\mathrm{k}$ This pathway also includes gliosarcoma.

variability in results across studies, many of these molecular markers are not yet widely used to subclassify gliomas, although the 2020 version of the WHO classification of CNS tumors will include $C D K N 2 A / B$ homozygous deletion as evidence of grade 4 status in $I D H$ mutant astrocytomas, as indicated by a recent consensus statement. ${ }^{24}$

\section{Prognostic Relevance of Molecular Subgroups in Glioma}

Numerous large studies of patients with brain tumors have determined that, among grade II-III gliomas, 1p19q codeletion correlates with greatly improved progressionfree survival (PFS) and overall survival (OS)..$^{3,7,8,25-27}$ Likewise, the presence of an $I D H$ mutation is a strong favorable prognostic marker for OS in grade II-III gliomas. ${ }^{4}$ Analyses within single treatment arms showed that the $I D H$ status is prognostic for outcome across a variety of postoperative adjuvant options. For example, in the NOA-04 phase III randomized trial in newly diagnosed anaplastic gliomas, IDH mutation was associated with improved PFS, longer time to treatment failure, and extended OS in each of the 3 treatment arms: standard RT $(n=160)$; combination therapy with procarbazine, lomustine, and vincristine (PCV; RT upon progression; $\mathrm{n}=78$ ); and temozolomide (TMZ; RT upon progression; $\mathrm{n}=80) .^{26}$

Multiple independent studies have shown that subdividing gliomas by molecular subtype, especially $I D H 1 / 2$ and $1 \mathrm{p} 19 \mathrm{q}$ status, yields greater prognostic separation than subdivision based on histology (as defined by WHO 2007). These include very large studies covering multiple grades and histology-based subtypes of gliomas, ${ }^{4,7,25}$ as well as smaller studies limited to 1 or 2 grades or histologic subtypes..$^{3,28-30}$ Multiple studies have also shown that, among patients with grade II-III gliomas, the $I D H$-mut plus 1p19q-codeletion group has the best prognosis, followed by $I D H$-mut without 1 p19q codeletion; the $I D H$-wt group has the worst prognosis. ${ }^{3-5,25-27}$ Analyses within single treatment arms have confirmed this trend in prognosis across a variety of postoperative adjuvant treatment options. . $^{3,26,27,30}$ 


\section{Anaplastic Gliomas ${ }^{\mathrm{a}} /$ Glioblastoma}

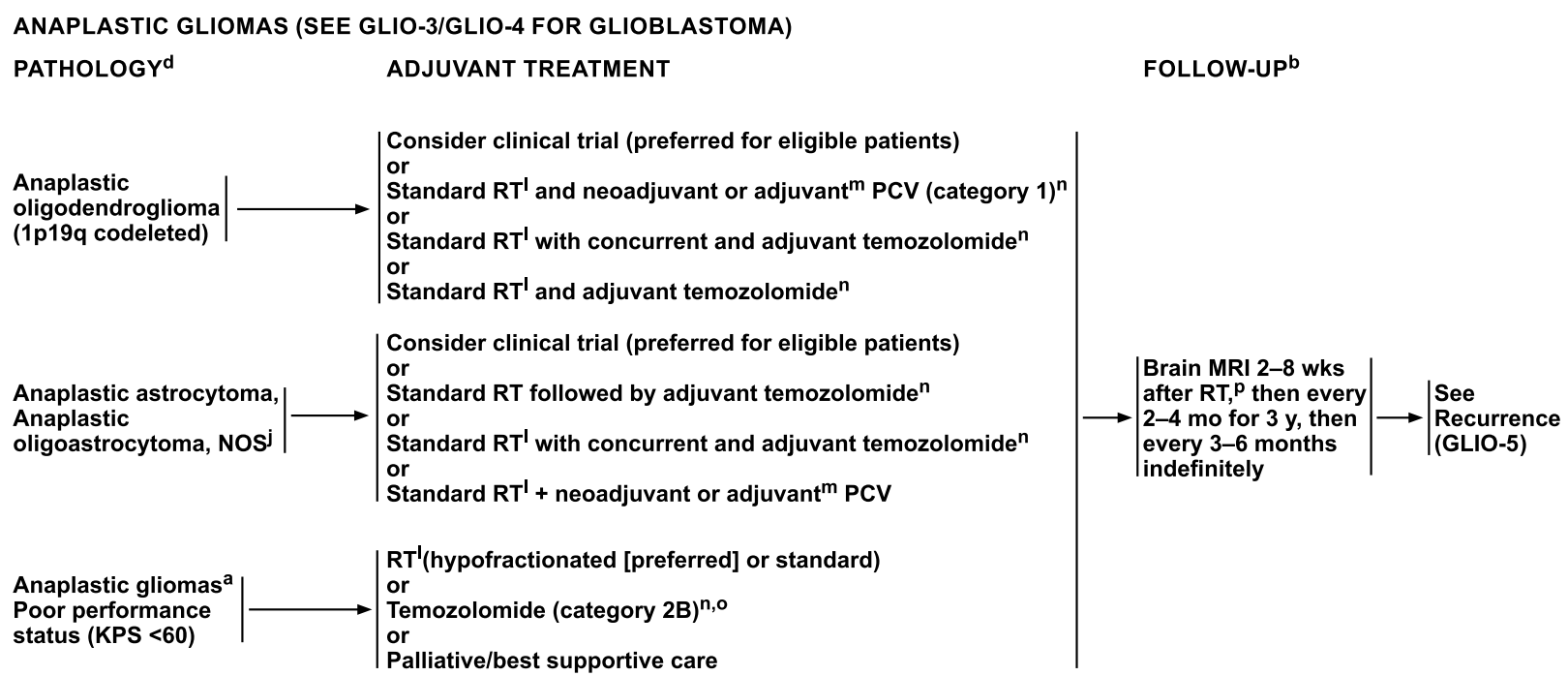

aThis pathway includes the classification of mixed $A O A, A A, A O$, and other rare anaplastic gliomas.

bSee Principles of Brain and Spine Tumor Imaging (BRAIN-A*).

dSee Principles of Brain Tumor Pathology (BRAIN-F*).

iThe 2016 WHO Classification of Tumors of the CNS has deleted oligoastrocytoma as a category, although "anaplastic oligoastrocytoma, NOS" may continue to be used for 1) patients with mixed histology and no available molecular data (ie, no tissue available for analysis) for determining whether to classify as oligodendroglioma versus astrocytoma; or 2) rare instances in which the tumor has regions with histologic features of oligoastrocytoma with $1 \mathrm{p} 19 \mathrm{q}$-codeletion, and distinct regions with features of oligoastrocytoma with $1 \mathrm{p} 19 \mathrm{q}-$-codeletion, and distinct
histologic features of astrocytoma without $1 \mathrm{p} 19 \mathrm{q}$-codeletion.

ISee Principles of Brain and Spinal Cord Tumor Radiation Therapy (BRAIN-C*). mThe panel recommends that PCV be administered after RT (as per EORTC 26951) since the intensive PCV regimen given prior to RT (RTOG 9402) was not tolerated as well.

nSee Principles of Brain and Spinal Cord Tumor Systemic Therapy (BRAIN-D).

${ }^{\circ}$ Consider temozolomide if tumor is MGMT promoter methylated.

PWithin the first 3 months after completion of RT and concomitant temozolomide, diagnosis of recurrence can be indistinguishable from pseudoprogression on neuroimaging.

${ }^{*}$ Available online, in these guidelines, at NCCN.org

TERT mutations in patients with high-grade $I D H$-wt glioma are associated with shorter OS, compared with $I D H$-wt tumors without a TERT mutation.,21,31 However, a multivariate analysis of data from 291 patients with IDH-mut+1p19q-codeleted oligodendrogliomas showed that absence of a TERT mutation was associated with worse OS, compared with patients with TERTmut oligodendrogliomas (HR, 2.72; 95\% CI, 1.05-7.04; $P=.04) .^{32}$

MGMT (O-6-methylguanine-DNA methyltransferase) is a DNA repair enzyme that can cause resistance to DNA-alkylating drugs. ${ }^{33}$ MGMT promoter methylation is associated with better survival outcomes in patients with high-grade glioma and is a predictive factor for response to treatment with alkylating chemotherapy such as TMZ or lomustine, ${ }^{22,34-36}$ even in older adult patients. ${ }^{37,38}$ Tumors with $H 3 K 27 M$ mutations are far less likely to be $M G M T$ promoter methylated ${ }^{23}$ and are associated with worse prognosis. ${ }^{39,40}$ Patients whose glioblastomas contain H3F3A G34 mutations however, may have relatively higher rates of $M G M T$ promoter methylation, and do not have a worse prognosis than other $I D H$-wt glioblastomas. ${ }^{40,41}$

Most pilocytic astrocytomas in pediatric patients contain $B R A F$ fusions or, less commonly, BRAF V600E mutations, especially those arising in the posterior fossa; such tumors are rarely high grade. ${ }^{42} B R A F$ fusion is associated with better prognosis in pediatric low-grade astrocytoma. $^{42-44}$ The likelihood of a BRAF fusion in a pilocytic astrocytoma decreases with age. ${ }^{42}$ The $B R A F$ V600E mutation is present in most pleomorphic xanthoastrocytomas, though it has also been found in some other pediatric low-grade gliomas, such as gangliogliomas and dysembryoplastic neuroepithelial tumors, ${ }^{22,42,45}$ as well as a small proportion of glioblastomas (especially epithelioid glioblastoma). ${ }^{46}$ Retrospective studies have shown that $B R A F$ V600E may be associated with increased risk of progression in pediatric low-grade gliomas, ${ }^{47}$ but one study found that this association was not quite statistically significant $(\mathrm{n}=198 ; P=.07) .{ }^{44}$ Some have shown that tumors with a BRAF V600E mutation may respond to BRAF inhibitors such as vemurafenib, ${ }^{48-50}$ but ongoing trials will 


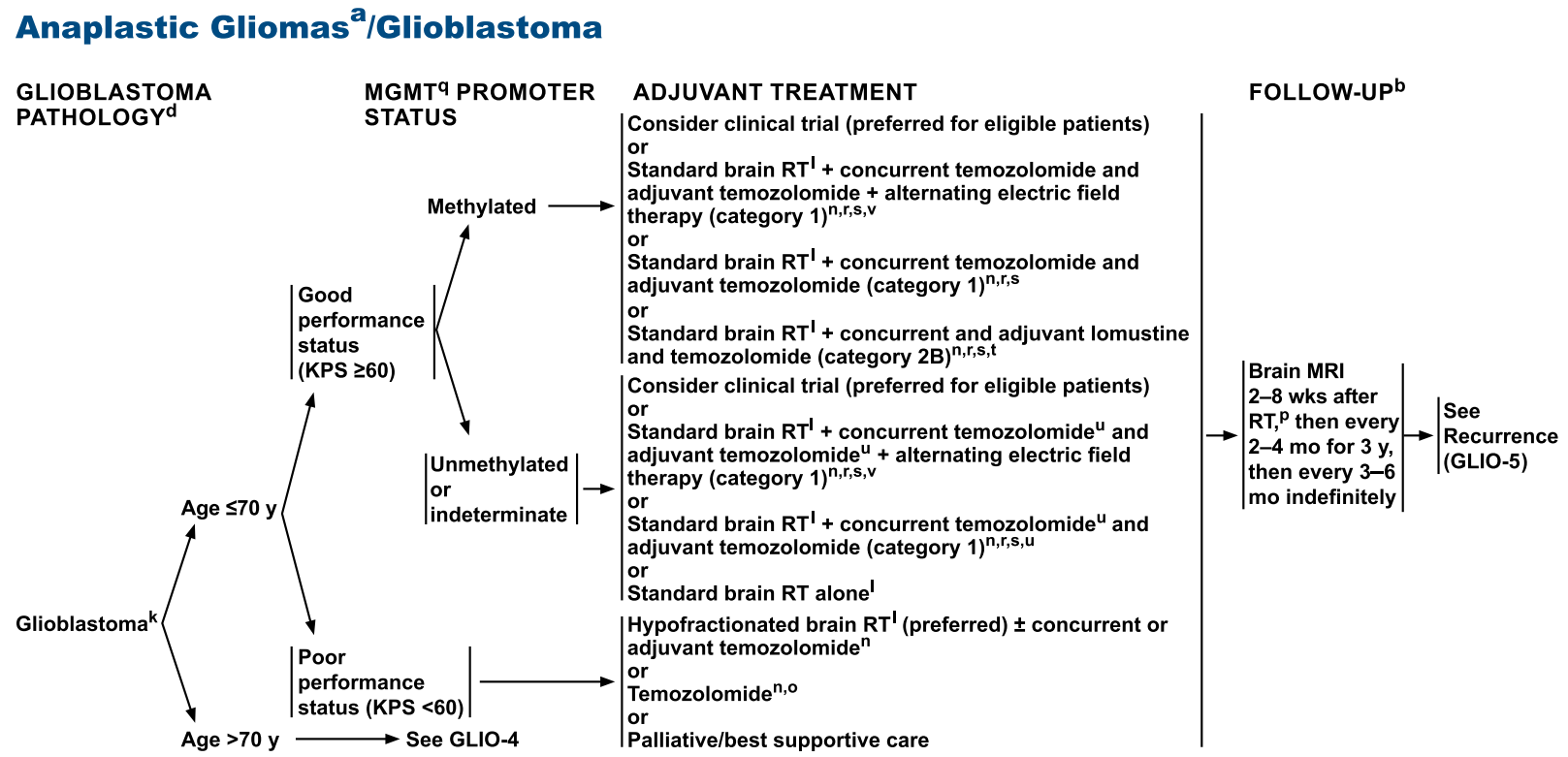

aThis pathway includes the classification of mixed $A O A, A A, A O$, and other rare anaplastic gliomas.

anaplastic gliomas.
bSee Principles of Brain and Spine Tumor Imaging (BRAIN-A*).

dSee Principles of Brain Tumor Pathology (BRAIN-F*).

kThis pathway also includes gliosarcoma.

ISee Principles of Brain and Spinal Cord Tumor Radiation Therapy (BRAIN-C*).

nSee Principles of Brain and Spinal Cord Tumor Systemic Therapy (BRAIN-D).

${ }^{\circ}$ Consider temozolomide if tumor is MGMT promoter methylated.

PWithin the first 3 months after completion of RT and concomitant temozolomide,

diagnosis of recurrence can be indistinguishable from pseudoprogression on neuroimaging.

9MGMT= O6-methylguanine-DNA methyltransferase

rCombination of modalities may lead to increased toxicity or radiographic changes. sBenefit of treatment with temozolomide for glioblastomas beyond 6 months is unknown.

tModerate to significant myelosuppression was observed, but the toxicity profile for this regimen is not yet fully defined.

uClinical benefit from temozolomide is likely to be lower in patients whose tumors

lack MGMT promoter methylation.

vAlternating electric field therapy is only an option for patients with supratentorial disease.

*Available online, in these guidelines, at NCCN.org

Version 3.2020, 04/30/20 ๑ National Comprehensive Cancer Network, Inc. 2020. All rights reserved

The NCCN Guidelines ${ }^{*}$ and this illustration may not be reproduced in any form without the express written permission of NCCN.

GLIO-3

further clarify targeted treatment options in the presence of a BRAF fusion or V600E mutation (eg, NCT03224767, NCT03430947). BRAF fusion and/or mutation testing are clinically indicated in patients with low-grade glioma.

\section{NCCN Molecular Testing Recommendations for Glioma}

Recommendations for molecular testing of glioma tumors are provided in the "Principles of Brain Tumor Pathology" section (Brain-F, available online, in these guidelines, at NCCN.org). Based on studies showing that IDH status is associated with better prognosis in patients with grade II-III glioma, ${ }^{14,25,26,51}$ the panel recommends IDH mutation testing in patients with glioma. Immunohistochemistry can detect the most common $I D H$ mutation, which is $I D H 1 \mathrm{R} 132 \mathrm{H}$. However, sequencing must be done to detect the less common $I D H 1$ mutations (eg, IDH1 R132C) and IDH2. This sequencing should be done in the proper clinical context (eg, younger patients with nonenhancing gliomas). Patients with oligodendroglioma should also undergo 1p19q testing. However, since $1 \mathrm{p} 19 \mathrm{q}$ codeletion is strongly associated with $I D H$ mutation, ${ }^{14,15,52}$ 1 p19q testing is not necessary in tumors that are definitely $I D H$-wt, and tumors without an $I D H$ mutation should not be regarded as $1 \mathrm{p} 19 \mathrm{q}$ codeleted, even when results suggest otherwise. Mutation testing for ATRX and TERT are also recommended, given the diagnostic value of these mutations. ${ }^{17,19-21}$ Screening for H3K27M mutations (H3F3A and HIST1H3B sequencing preferred) and BRAF fusion and/or mutation testing may be carried out as clinically indicated.

Grade III-IV gliomas should undergo testing for MGMT promoter methylation status, since MGMT promoter methylated tumors typically respond better to alkylating chemotherapy, compared with unmethylated tumors. ${ }^{34,37,38,53}$ To date, there are no targeted agents that have shown improvement in OS in the treatment of glioblastoma. Nevertheless, molecular testing of glioblastomas is still encouraged by the panel, as patients with a detected driver mutation may be treated with a targeted therapy on a compassionate use basis, and these tests improve diagnostic accuracy and prognostic stratification. 


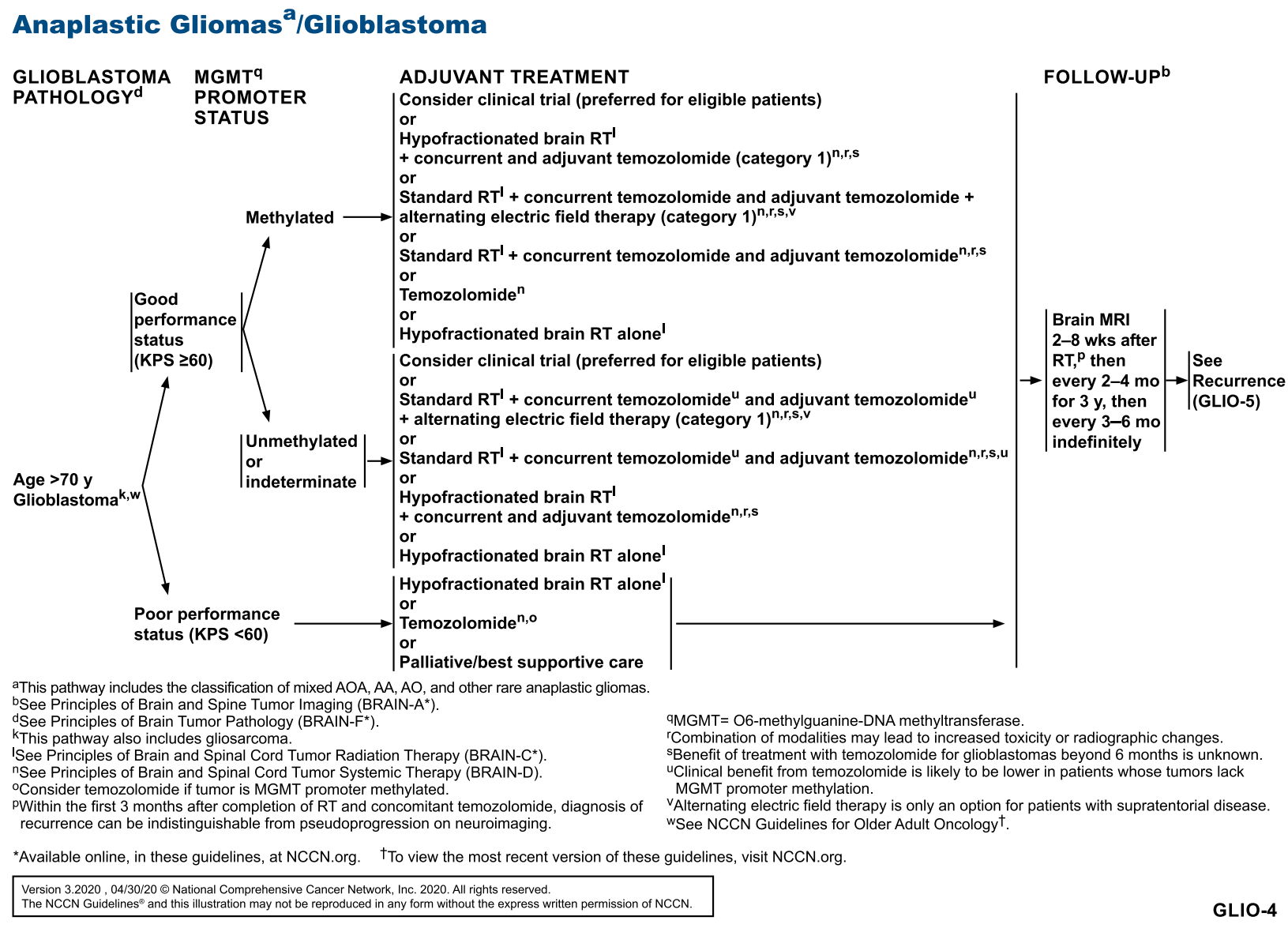

Detection of genetic or epigenetic alterations could also expand clinical trial options for a brain tumor patient.

\section{Low-Grade Gliomas}

Low-grade gliomas (ie, pilocytic and diffusely infiltrative astrocytomas, oligodendrogliomas) are a diverse group of relatively uncommon malignancies classified as grade I and II under the WHO grading system. ${ }^{1}$ Low-grade gliomas comprise approximately $5 \%-10 \%$ of all CNS tumors. ${ }^{54}$ Seizure is a common symptom (81\%) of lowgrade gliomas, and is more frequently associated with oligodendrogliomas. ${ }^{55,56}$ The median duration from onset of symptoms to diagnosis ranges from 6 to 17 months.

\section{Grade I Gliomas}

Diffuse astrocytomas are poorly circumscribed and invasive, and most gradually evolve into higher-grade astrocytomas. Although these were traditionally considered benign, they can behave aggressively and will undergo anaplastic transformation within five years in approximately half of patients. ${ }^{57,58}$ The most common noninfiltrative astrocytomas are pilocytic astrocytomas. Other grade I gliomas in which treatment recommendations are included in the NCCN Guidelines for CNS Cancers are pleomorphic xanthoastrocytoma, subependymal giant cell astrocytoma (SEGA), and ganglioglioma, though these grade I gliomas are uncommon. Pleomorphic xanthoastrocytomas are associated with favorable prognosis, ${ }^{59,60}$ though mitotic index is associated with survival outcomes. ${ }^{60,61}$ Gangliogliomas are commonly located in the temporal lobe, and the most significant predictors of survival are low tumor grade and younger age. ${ }^{62}$

SEGAs are typically located at the caudothalamic groove adjacent to the foramen of Monro. Though they are generally slow-growing and histologically benign, they can also be associated with manifestations such as hydrocephalus, intracranial pressure, and seizures. ${ }^{63}$ SEGAs can be distinguished from subependymal nodules by their characteristic serial growth. ${ }^{64}$ These tumors occur in $5 \%-20 \%$ of individuals with tuberous sclerosis complex (TSC). ${ }^{65-67}$

\section{Treatment}

Grade I gliomas are usually curable by surgery alone. Indication for treatment of SEGAs is based on development 


\section{Anaplastic Gliomas ${ }^{\mathrm{a}} / \mathrm{Glioblastoma}$}

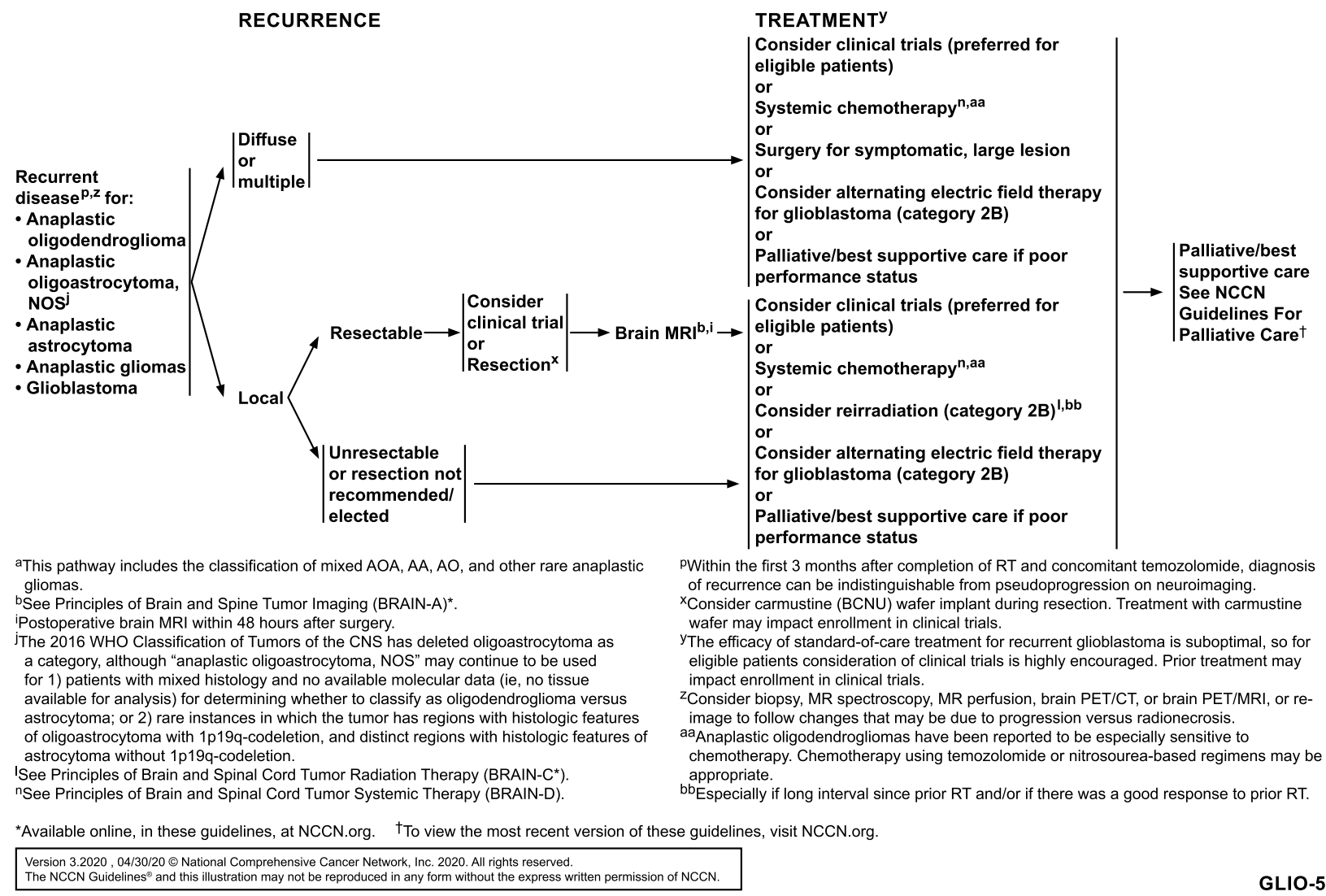

of new symptoms or radiologic evidence of tumor growth. ${ }^{64}$ Though surgery is sometimes a recommended option for SEGAs, many are in an area not amenable to resection, and recurrence may occur following resection. ${ }^{68,69}$ Surgery may pose risks because of the frequent location of SEGAs near the foramen of Monro, but in specialized centers, morbidity is acceptable, and surgical mortality is extremely low. ${ }^{70}$

There is some evidence that BRAF inhibitors, as well as a BRAF/MEK inhibitor combination, may be used for treatment of low-grade gliomas that are BRAF mutated. The phase II VE-BASKET study showed that vemurafenib was efficacious in BRAF-mutated low-grade gliomas, particularly PXA, with an ORR of $42.9 \%(n=7)$, median PFS of 5.7 months, and median OS not reached. ${ }^{50}$ Another phase II trial including 10 patients with low-grade glioma showed that dabrafenib/trametinib was associated with an ORR of $56 \%$ (5 patients with a partial response and 4 patients with stable disease).${ }^{71}$ Case reports have demonstrated clinical activity for the combination BRAF/MEK inhibitor dabrafenib/trametinib in patients with BRAF V600E mutant glioma. ${ }^{72,73}$
Reducing or stabilizing the volume of SEGAs through systemic therapy has been investigated. A phase III trial showed that 78 patients with SEGA and TSC who received everolimus, an mTOR inhibitor, had at least a $50 \%$ reduction in tumor volume, compared with 39 patients who received a placebo $(35 \%$ vs $0 \% ; P<.001)$, and 6 -month PFS was $100 \%$ versus $86 \%$, respectively $(P<.001) .{ }^{74}$ Analyses from a long-term follow-up showed that median duration of response was not reached, with response duration ranging from 2.1 months to 31.1 months. ${ }^{75}$ Tumor volume reduction rates of $30 \%$ and $50 \%$ were maintained in patients in the everolimus arm for more than 3 years. This regimen was generally well-tolerated, with the most frequently reported grade 3 or 4 adverse events being stomatitis (8\%) and pneumonia (8\%). Everolimus has also been investigated in a phase II trial including 58 patients with recurrent grade II gliomas, with a 6-month PFS rate of $84 \% .{ }^{76}$ Medical therapy of SEGA, while effective, is a long-term commitment, unless it is being used short-term to facilitate surgical resection. Once mTOR 


\section{Limited Brain Metastases}

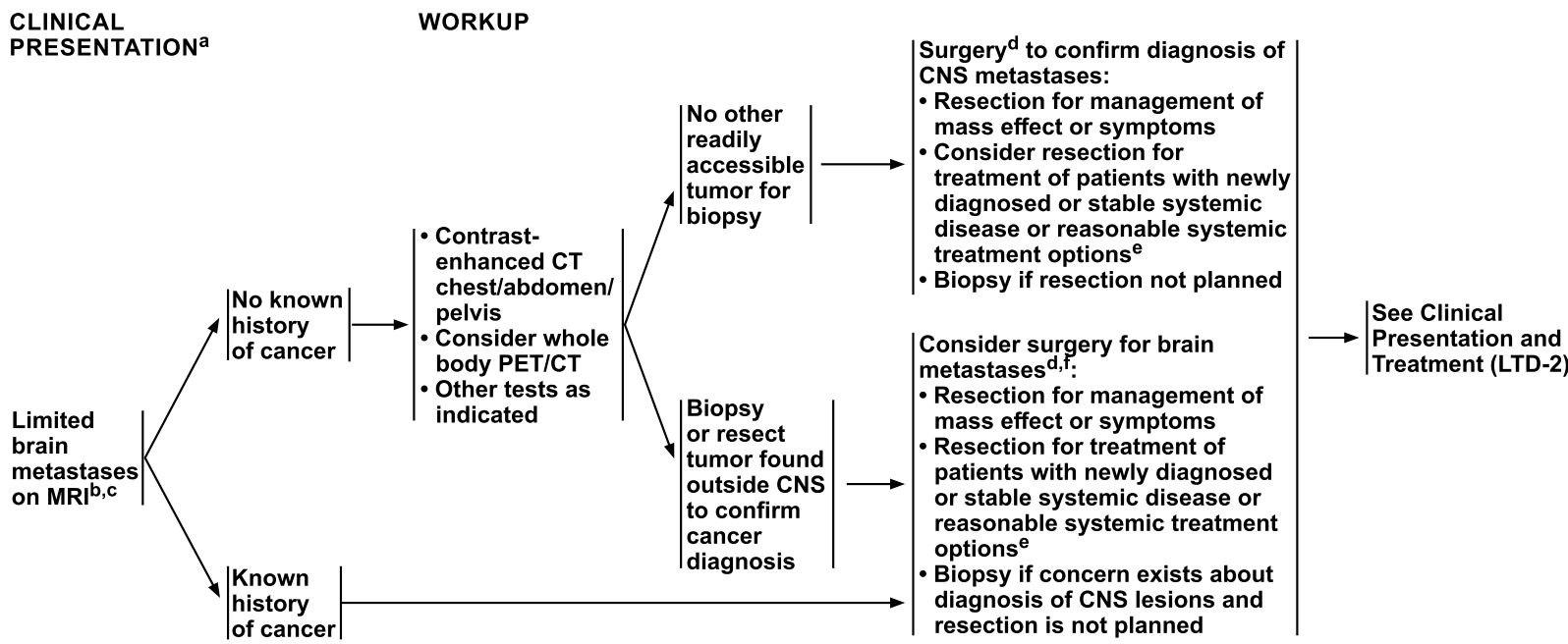

aSee Principles of Brain and Spine Tumor Imaging (BRAIN-A*).

${ }^{b}$ Consider a multidisciplinary review in treatment planning, especially once pathology is available. See Principles of Brain and Spine Tumor Management (BRAIN-E*)

c"Limited" brain metastases defines a group of patients for whom SRS is equally effective and offers significant cognitive protection compared with WBRT. The definition of "limited" brain metastases in terms of number of metastases or tota intracranial disease volume is evolving and may depend on the specific clinical situation. (Yamamoto M, Serizawa T, Shuto T, et al. Stereotactic radiosurgery prospective observational study. Lancet Oncol 2014;15:387-395.)
dSee Principles of Brain Tumor Surgery (BRAIN-B*)

eFor secondary CNS lymphoma, treatment may include systemic treatment, whole-brain or focal RT, or combination.

fThe decision to resect a tumor may depend on the need to establish histologic
fThole-brain or focal diagnosis, the size of the lesion, its location, and institutional expertise. For example, smaller $(<2 \mathrm{~cm})$, deep, asymptomatic lesions may be considered for treatment with SRS versus larger $(>2 \mathrm{~cm})$, symptomatic lesions that may be more appropriate for surgery. (Ewend MG, Morris DE, Carey LA, Ladha AM, Brem S. Guidelines for the initial management of metastatic brain tumors: role of surgery, radiosurgery, and radiation therapy. J Natl Compr Cancer Netw 2008;6:505-513.)

*Available online, in these guidelines, at NCCN.org.

inhibitor therapy is stopped, lesions typically recur, usually within several months, and eventually reach pretreatment volume. The lesions will continue to grow unless therapy is reintroduced. Most patients tolerate long-term therapy with mTOR inhibitors quite well. ${ }^{77}$

\section{NCCN Recommendations}

When possible, maximal safe resection is recommended for grade I gliomas, and the actual extent of resection should be documented with a T2-weighted or FLAIR MRI scan within 48 hours after surgery. Patients may be observed following surgery. If incomplete resection or biopsy, or if surgery was not feasible, then RT may be considered if there is significant tumor growth or if neurologic symptoms are present or develop. A BRAF/ MEK inhibitor combination may be used for patients with BRAF V600E mutant low-grade glioma. Treatment with an mTOR inhibitor (eg, everolimus) should be considered for patients with SEGA, ${ }^{74,75}$ though institutional expertise and patient preference should guide treatment decision-making for these rare tumors. ${ }^{64}$ Full treatment recommendations can be found on ASTR-1 (page 1538).

\section{Grade II Infiltrative Supratentorial Astrocytoma/ Oligodendroglioma}

Radiographically, low-grade oligodendrogliomas appear well demarcated, occasionally contain calcifications, and do not often enhance with contrast. In histology, the typical "fried egg" appearance of these tumors is evident as a fixation artifact in paraffin but not in frozen sections. Grade II oligodendrogliomas have a much better 5 -year survival rate $(82.7 \%)$ than diffuse astrocytomas (51.6\%). ${ }^{78}$

Factors prognostic for PFS or OS in patients with grade II gliomas include age, tumor diameter, tumor crossing midline, neurologic or performance status (PS) prior to surgery, and the presence of certain molecular markers (see "Molecular Profiling for Gliomas," page 1538).,8,79-84 For example, IDH1/2 mutation is associated with a favorable prognosis in patients with grade II and III gliomas, ${ }^{4,5,26}$ supporting the emerging idea that molecular analysis should play a 


\section{Limited Brain Metastases}

CLINICAL PRESENTATION

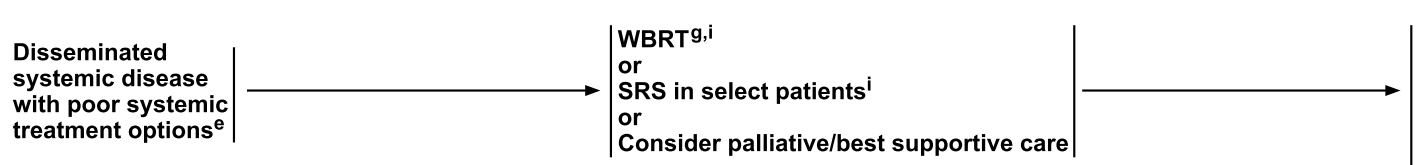

eFor secondary CNS lymphoma, treatment may include systemic treatment, whole-brain or focal RT, or combination.

gIf an active agent exists (eg, cytotoxic, targeted, or immune modulating), trial of systemic therapy with good CNS penetration may be considered in select patients (eg, for patients with small asymptomatic brain metastases from melanoma or ALK rearrangement-positive NSCLC or EGFR-mutated NSCLC); it is reasonable to hold on treating with radiation to see if systemic therapy can control the brain metastases. Consultation with a radiation oncologist and close MRI surveillance is strongly recommended. There are no data from prospective clinical trials comparing the two strategies to assess what the impact of delayed radiation would be in terms of survival or in delay of neurologic deficit development.

h See Principles of Brain and Spinal Cord Tumor Systemic Therapy (BRAIN-D).

isee Principles of Brain and Spinal Cord Tumor Radiation Therapy (BRAIN-C*)

jSRS is preferred when safe, especially for low tumor volume, to both the resection cavity and any other non-resected brain metastases. WBRT is generally not recommended but may be appropriate in some rare clinical circumstances.

kFor brain metastases not managed with resection, SRS + WBRT is generally not recomended but may be appropriate in some rare clinical circumstances. Brown 2016 showed that for tumors $<3 \mathrm{~cm}$, SRS + WBRT improved local control compared with SRS alone, but did not significantly improve survival, and was associated with greater cognitive decline and poorer quality of life. (Brown PD, Jaeckle K, Ballman KV, et al. Effect of radiosurgery alone vs radiosurgery with whole brain radiation therapy on cognitive function in patients with 1 to 3 brain metastases: a randomized clinical trial. JAMA 2016;316:401-409.)

'Hippocampal avoidance preferred. See BRAIN-C*.

*Available online, in these guidelines, at NCCN.org

Version 32020 , 04/30/20 @ National Comprehensive Cancer Network Inc 2020. All rights reserved.

The NCCN Guidelines ${ }^{\mathbb{E}}$ and this illustration may not be reproduced in any form without the express written permission of NCCN.

larger role in treatment decision-making, relative to histopathology. ${ }^{56}$

\section{Treatment Overview}

\section{Surgery}

Surgery remains an important diagnostic and therapeutic modality. The primary surgical goals are maximal safe resection to delay progression and improve survival, relief of symptoms, and provision of adequate tissue for a pathologic diagnosis and grading. Needle biopsies are often performed when lesions are in deep or critical regions of the brain. Biopsy results can be misleading, because gliomas often have varying degrees of cellularity, mitoses, or necrosis from one region to another; thus, small samples can provide erroneous histologic grade or diagnosis. ${ }^{85,86}$

Surgical resection plays an important role in the management of low-grade gliomas. A systematic review showed that gross total resection (GTR) was significantly associated with decreased mortality and lower risk of disease progression up to 10 years after treatment, compared with STR. ${ }^{87}$ Because these tumors are relatively uncommon, published series generally include patients treated for decades, which introduces additional variables. For example, the completeness of surgical excision was based on the surgeon's report in older studies. This approach is relatively unreliable when compared with assessment by modern postoperative imaging studies. Furthermore, many patients also received RT, and thus the net effect of the surgical procedure on outcome is difficult to evaluate. Two meta-analyses including studies of primary lowgrade gliomas show that extent of resection is a significant prognostic factor for PFS and/or OS. ${ }^{88,89}$ Maximal safe resection may also delay or prevent malignant progression ${ }^{89-91}$ and recurrence. ${ }^{92}$ Patients who undergo an STR, open biopsy, or stereotactic biopsy are, therefore, considered to be at higher risk for progression. Gross total resection is also associated with improved seizure control compared with subtotal resection. $^{89}$

Biologic considerations also favor an attempt at a complete excision of a low-grade glioma. First, the tumor may contain higher-grade foci, which may not be 


\section{Limited Brain Metastases}

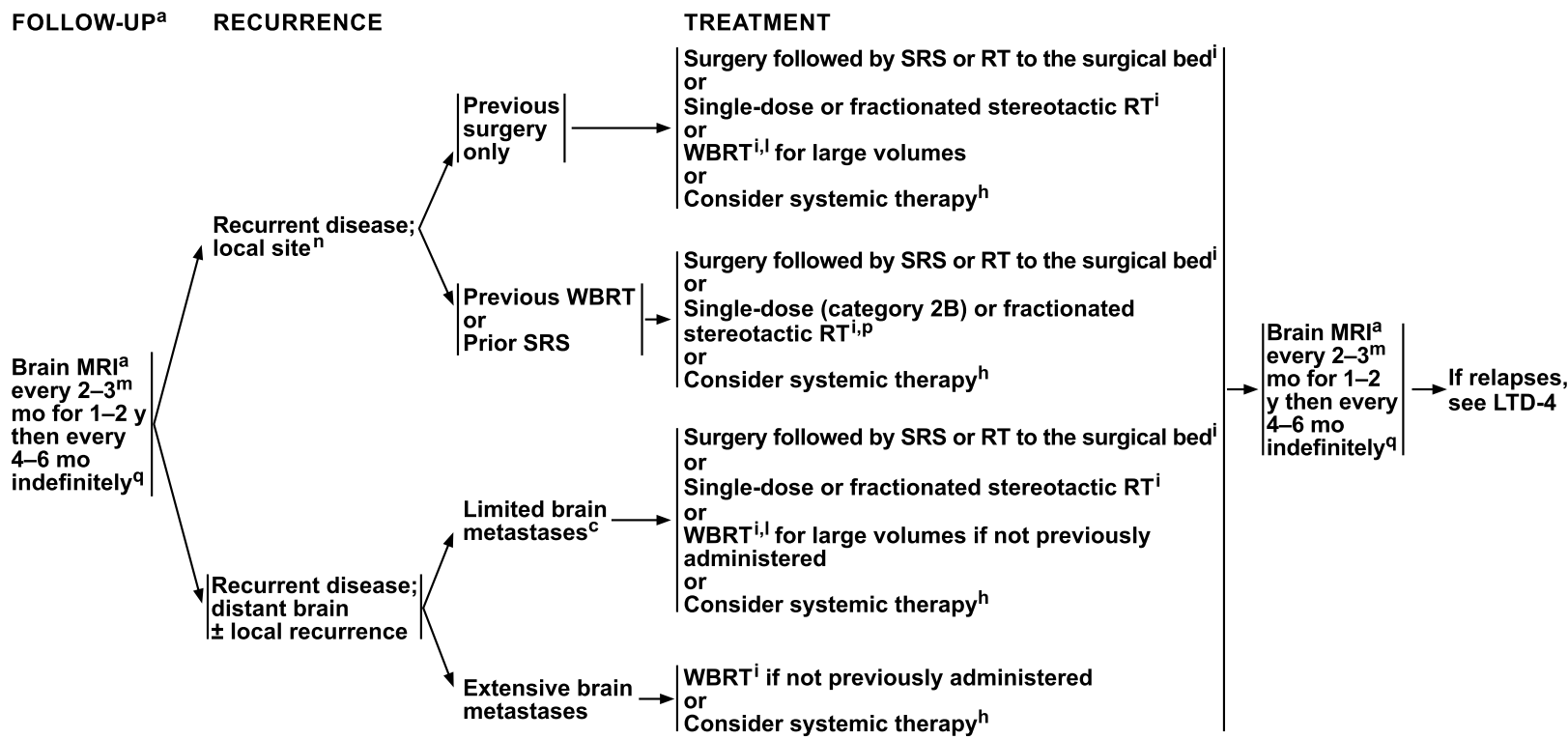

aSee Principles of Brain and Spine Tumor Imaging (BRAIN-A*).

C"Limited" brain metastases defines a group of patients for whom SRS is equally effective and offers significant cognitive protection compared with WBRT. The definition of "limited" brain metastases in terms of number of metastases or total intracranial disease volume is evolving and may depend on the specific clinical situation. (Yamamoto M, Serizawa T, Shuto T, et al. Stereotactic radiosurgery for patients with multiple brain metastases (JLGK0901): a multi-institutional prospective observational study. Lancet Oncol 2014;15:387-395.)

hSee Principles of Brain and Spinal Cord Tumor Systemic Therapy (BRAIN-D).

iSee Principles of Brain and Spinal Cord Tumor Radiation Therapy (BRAIN-C*). 'Hippocampal avoidance preferred. See BRAIN-C*

mMRI every 2 months (instead of $3 \mathrm{mo}$ ) for those patients treated with SRS alone. nAfter SRS, recurrence on MRI can be confounded by treatment effects; consider tumor tissue sampling if there is a high index of suspicion of recurrence.

PIf patient had previous SRS with a good response $>6 \mathrm{mo}$, then reconsider SRS if imaging supports active tumor and not necrosis.

q/maging to evaluate emergent signs/symptoms is appropriate at any time.

*Available online, in these guidelines, at NCCN.org.

reflected in a small specimen. Second, complete excision may decrease the risk of future dedifferentiation to a more malignant tumor. ${ }^{93}$ Third, removal of a large tumor burden may enhance the benefit of RT. As a result of these considerations, the general recommendation for treating a low-grade glioma is to first attempt as complete an excision of tumor as possible (based on postsurgical MRI verification) without compromising function. However, for tumors that involve eloquent areas, a total removal may not be feasible, and an aggressive approach could result in neurologic deficits. Residual tumor volume may also be a prognostic factor, with a randomized single institution study showing that the OS benefit of maximal safe resection was limited to patients with a residual tumor volume $<15 \mathrm{~cm}^{3} .^{94}$

\section{Adjuvant Therapy}

A large meta-analysis, including data from phase 3 trials (EORTC 22844 and 22845,95,96 and NCCTG 86-72-5182), confirmed that surgery followed by RT significantly improves PFS but not OS in patients with low-grade gliomas. ${ }^{97}$ Early versus late postoperative RT did not significantly affect OS, however, suggesting that observation is a reasonable option for some patients with newly diagnosed gliomas. ${ }^{96}$

Final results of a phase 3 randomized clinical trial, RTOG 9802, which assessed the efficacy of adjuvant RT versus RT followed by 6 cycles of PCV in patients with newly diagnosed supratentorial WHO grade II gliomas and at least one of 2 risk factors for disease progression (STR or age $\geq 40$ years) ${ }^{98}$ showed significant improvements in both PFS and OS with the addition of PCV. ${ }^{99}$ The median survival time increased from 7.8 years to 13.3 years $(P=.02)$, and the 10 -year survival rate increased from $41 \%$ to $62 \%$. It is important to note, however, that roughly three-quarters of the study participants had a Karnofsky Performance Status (KPS) score of 90 to 100, and the median age was around 40 years. ${ }^{98}$ Exploratory analyses based on histologic subgroups showed a statistically significant improvement in OS for all subgroups except for patients with astrocytoma. ${ }^{99}$ Given that the study participants treated with PCV after RT experienced a significantly higher incidence of grade 3 or 4 adverse events (specifically neutropenia, gastrointestinal disorder, 


\section{Limited Brain Metastases}

RECURRENCE

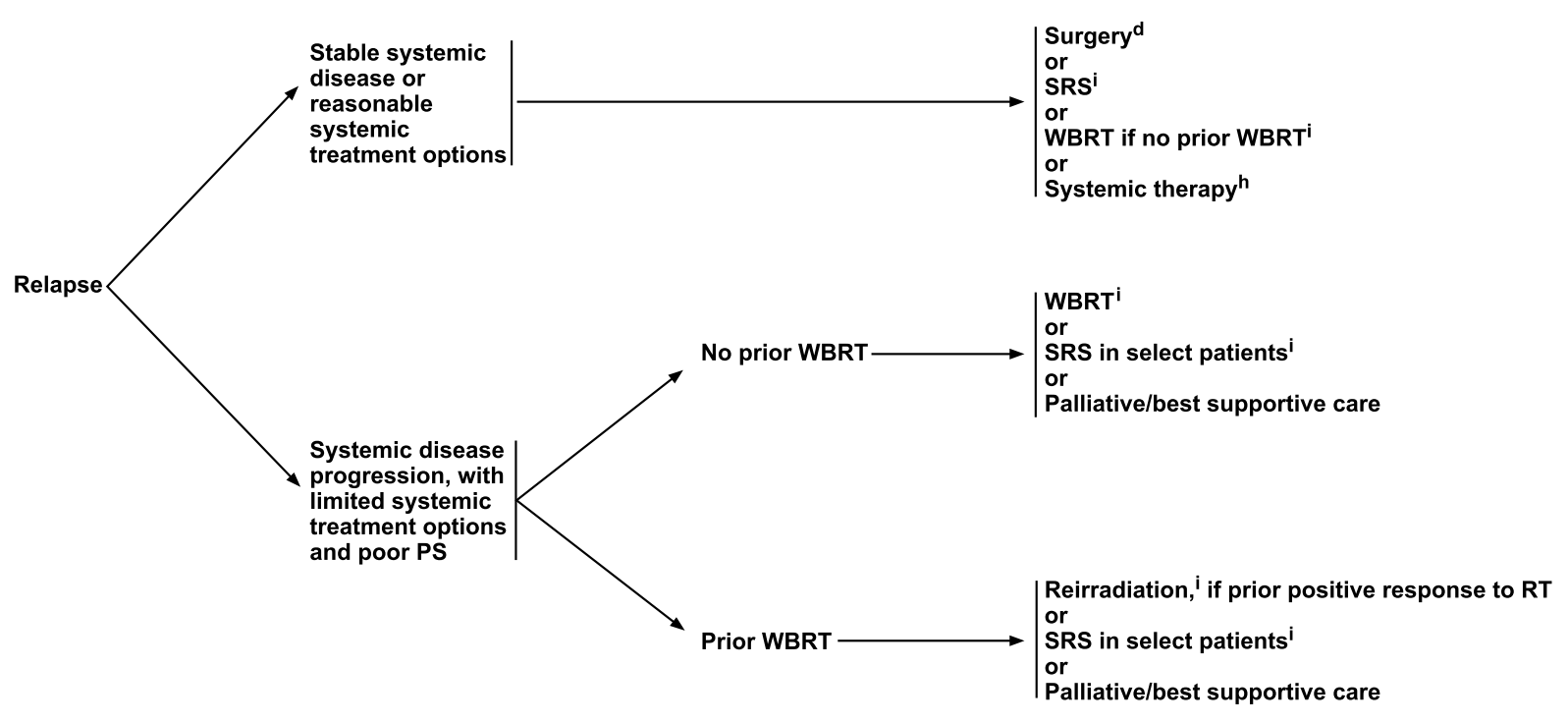

dSee Principles of Brain Tumor Surgery (BRAIN-B*).

iSee Principles of Brain and Spinal Cord Tumor Radiation Therapy (BRAIN-C*).

hSee Principles of Brain and Spinal Cord Tumor Systemic Therapy (BRAIN-D).

*Available online, in these guidelines, at NCCN.org

Version 3.2020, 04/30/20 @ National Comprehensive Cancer Network, Inc. 2020. All rights reserved.

The NCCN Guidelines ${ }^{\otimes}$ and this illustration may not be reproduced in any form without the express written permission of NCCN.

and fatigue), ${ }^{98,99}$ PCV may be difficult to tolerate in patients who are older or with poor PS. A retrospective subgroup analysis suggest that the survival benefit with the addition of PCV was seen only in $I D H$-mut tumors; the $I D H$-wt subgroup did not appear to benefit from the chemotherapy. ${ }^{100}$

Combined treatment with RT plus TMZ is supported by a phase 2 multicenter trial (RTOG 0424) in patients with supratentorial WHO grade II tumors and additional risk factors (ie, age $\geq 40$ years, astrocytoma, bihemispherical, tumor diameter $\geq 6 \mathrm{~cm}$, neurologic function status $>1) .{ }^{101}$ However, since the historical controls included patients treated in an earlier time period using different RT protocols, prospective controlled trials are needed to determine whether treatment with TMZ concurrently and following RT is as efficacious as PCV following radiation. There are currently no phase III data to support the use of RT and TMZ over RT and PCV for the treatment of patients with newly diagnosed high-risk low-grade glioma. The phase 3 randomized EORTC 22033-26033 trial showed that PFS is not significantly different for adjuvant RT versus dose-dense TMZ in patients with resected or biopsied supratentorial grade II glioma and more than one risk factor $(n=477) .{ }^{9}$ However, analyses of OS have not yet been reported for this trial.

\section{Radiation Therapy}

When RT is given to patients with low-grade gliomas, it is administered with restricted margins. A T2-weighted (occasionally enhanced T1) and/or FLAIR MRI scan is the best means for evaluating tumor extent, because these tumors enhance weakly or not at all. The clinical target volume (CTV) is defined by the FLAIR or T2-weighted tumor with a 1- to 2-cm margin. Every attempt should be made to decrease the RT dose outside the target volume. This can be achieved with 3-dimensional planning or intensity-modulated RT (IMRT), with improved target coverage and normal brain/critical structure sparing often shown with IMRT. ${ }^{102,103}$ The recommended dosing for postoperative RT is based on results from two phase 3 randomized trials showing that higher dose RT had no significant effect on OS or time to progression, ${ }^{82,95}$ and on several retrospective analyses showing similar results. ${ }^{81,83,104}$ Because higher doses offer no clear advantages, the NCCN 
Extensive Brain Metastases

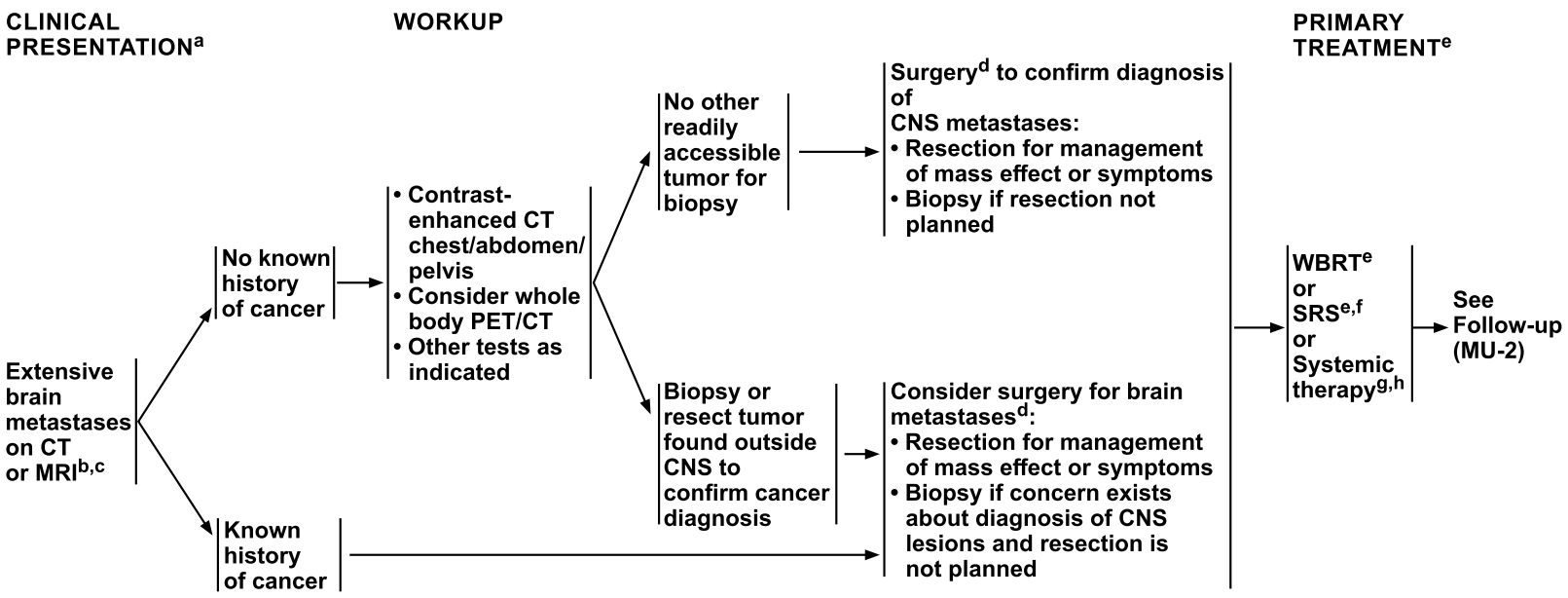

asee Principles of Brain and Spine Tumor Imaging (BRAIN-A*)

${ }^{b}$ Consider a multidisciplinary review in treatment planning, especially once pathology is available. See Principles of Brain and Spine Tumor Management (BRAIN-E) CIncludes all cases that do not fit the definition of "limited brain metastases" on LTD-1.

dSee Principles of Brain Tumor Surgery (BRAIN-B*).

esee Principles of Brain and Spinal Cord Tumor Radiation Therapy (BRAIN-C*).

fSRS can be considered for patients with good performance and low overall tumor volume and/or radioresistant tumors such as melanoma. (Yamamoto M, Serizawa

T, Shuto T, et al. Stereotactic radiosurgery for patients with multiple brain metastases (JLGK0901): a multi-institutional prospective observational study. Lancet Oncol 2014;15:387-395.)

gIf an active agent exists (eg, cytotoxic, targeted, or immune modulating), trial of systemic therapy with good CNS penetration may be considered in select patients (eg, for patients with small asymptomatic brain metastases from melanoma or ALK rearrangement-positive NSCLC or EGFR-mutated NSCLC); it is reasonable to hold on treating with radiation to see if systemic therapy can control the brain metastases. Consultation with a radiation oncologist and close MRI surveillance is strongly recommended. There are no data from prospective clinical trials comparing the two strategies to assess what the impact of delayed radiation would be in terms of survival or in delay of neurologic deficit development.

hSee Principles of Brain and Spinal Cord Tumor Systemic Therapy (BRAIN-D).

${ }^{*}$ Available online, in these guidelines, at $\mathrm{NCCN}$.org.

CNS Panel recommends lower-dose RT (45-54 Gy) for treatment of low-grade gliomas (grades I/II), including high-risk cases. However, $I D H$-wt low-grade gliomas have similar survival only slightly better than $I D H$-wt glioblastomas. ${ }^{4}$ Therefore, an RT dose of 59.4 to 60 Gy may be considered for this subset of patients with low-grade glioma. Preliminary data suggests that proton therapy could reduce the radiation dose to developing brain tissue and potentially diminish toxicities without compromising disease control. ${ }^{105}$

\section{Recurrent or Progressive Disease}

Though the survival impact is unclear, surgery for recurrent disease in patients with low-grade glioma may reduce symptoms, provide tissue for evaluation, and potentially allow for molecular characterization of the tumor. ${ }^{106-109}$ Maximal safe resection could play an important role for optimizing survival outcomes; a threshold value is unknown, but $>90 \%$ extent of resection is suggested. ${ }^{109}$ For patients without previous RT, results of the RTOG 9802 trial $^{98,99}$ support use of chemotherapy with RT. Data from phase II trials inform recommendations for chemotherapy treatment of patients with recurrent or progressive low-grade glioma. ${ }^{110-115} \mathrm{~Pa}$ tients should be enrolled in clinical trials evaluating systemic therapy options.

\section{NCCN Recommendations}

\section{Primary and Adjuvant Treatment}

For treatment recommendations for newly diagnosed grade II gliomas, the panel used the RTOG $9802^{98,99}$ criteria for determining if a patient is considered to be at low or high risk for tumor progression: patients are categorized as being at low risk if they are 40 years or younger and underwent a GTR; high-risk patients are older than 40 years of age and/or underwent an STR. However, the panel acknowledges that other prognostic factors have been used to guide adjuvant treatment choice in other studies of patients with low-grade glioma, ${ }^{116}$ such as tumor size, presence of neurologic deficits, loss of $C D K N 2 A$ homozygous deletion and the $I D H$ mutation status of the tumor..$^{9,79}$ If these other risk factors are considered, and treatment of a patient is warranted, 


\section{Extensive Brain Metastases}

FOLLOW-UPa

RECURRENCE

TREATMENT

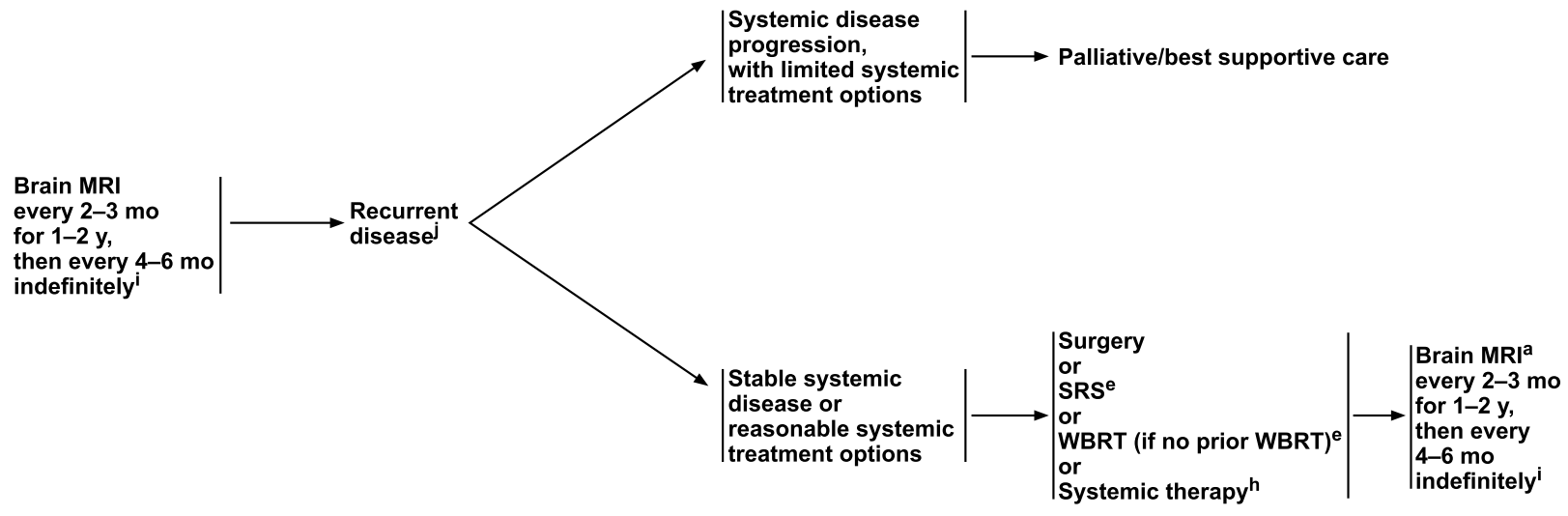

\footnotetext{
asee Principles of Brain and Spine Tumor Imaging (BRAIN-A*).

eSee Principles of Brain and Spinal Cord Tumor Radiation Therapy (BRAIN-C*).

hSee Principles of Brain and Spinal Cord Tumor Systemic Therapy (BRAIN-D).

iImaging to evaluate emergent signs/symptoms is appropriate at any time.

jAfter SRS, recurrence on MRI can be confounded by treatment effects; consider tumor tissue sampling if there is a high index of suspicion of recurrence.

*Available online, in these guidelines, at NCCN.org.

then the panel recommends that the patient be treated as high-risk.

Patients with low-risk and low-grade glioma may be observed following surgery. Close follow-up is essential as over half of these patients will develop tumor progression within 5 years. ${ }^{84}$ Following surgery, RT followed by PCV is a category 1 recommendation for patients with grade II glioma who are considered to be at high risk for tumor progression, based on the practice-changing results from the RTOG 9802 study, ${ }^{98,99}$ as discussed above. There is currently a lack of prospective randomized phase 3 data for the use of radiation and TMZ in patients with low-grade glioma, but interim data from the phase III CATNON trial illustrate that there is a benefit from adjuvant TMZ in patients with newly diagnosed $1 \mathrm{p} 19 \mathrm{q}$ noncodeleted anaplastic gliomas. ${ }^{117}$ Therefore, RT followed by adjuvant TMZ is a category $2 \mathrm{~A}$ option. Data from EORTC and NCIC studies, which included patients with glioblastoma, support RT with concurrent and adjuvant TMZ as an evidencebased regimen. ${ }^{118,119}$ Therefore, this is also a category $2 \mathrm{~A}$ option. Because PCV is generally a more difficult chemotherapy regimen to tolerate than TMZ, it may be reasonable to treat an elderly patient or a patient with multiple comorbidities with RT and TMZ instead of RT and PCV, but there are currently no data to show that doing so would result in similar improvement in OS.

Since the design of RTOG $9802^{98,99}$ did not address whether all patients should be treated with RT followed by PCV immediately after a tissue diagnosis (an observation arm was not included for patients with high-risk glioma [defined as are older than 40 years of age and/or underwent an STR $]^{84}$ in the study), observation after tissue diagnosis may be a reasonable option for some patients with high-risk grade II glioma who are neurologically asymptomatic or who have stable disease. However, close monitoring of such patients with brain MRI is important. Results from EORTC 22845, which showed that treatment with RT at diagnosis versus at progression did not significantly impact OS, provide rationale for observation in select cases with low-grade gliomas as an initial approach, deferring RT. ${ }^{96}$ Long-term toxicity from radiation needs to be a consideration, especially for young patients with $1 \mathrm{p} 19 \mathrm{q}$ codeletion, for whom there is slightly higher risk of radiation necrosis. ${ }^{120}$ 


\section{Central Nervous System Cancers}

PRINCIPLES OF BRAIN AND SPINAL CORD TUMOR SYSTEMIC THERAPY

ADULT LOW-GRADE GLIOMA/PILOCYTIC AND INFILTRATIVE SUPRATENTORIAL ASTROCYTOMA/OLIGODENDROGLIOMA

\begin{tabular}{|c|c|c|c|}
\hline & Preferred Regimens & Other Recommended Regimens & Useful in Certain Circumstances \\
\hline Adjuvant Treatment & $\begin{array}{l}\text { - RT + adjuvant PCV } \\
\text { (category } 1)^{1,2} \\
\cdot \text { RT + concurrent and adjuvant } \\
\text { temozolomide } \\
\cdot \text { RT + adjuvant temozolomide } \\
\text { R-5 }\end{array}$ & $\begin{array}{l}\text { Temozolomide } \\
\text { - PCV } \text { PC }^{\mathrm{b}, 3,4}\end{array}$ & 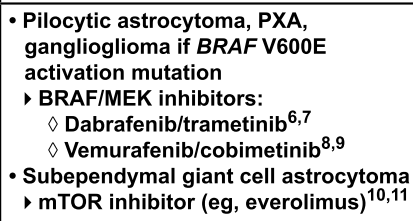 \\
\hline Recurrent or Progressive Disease ${ }^{a}$ & - None & $\begin{array}{l}\text { - RT + adjuvant PCV } \\
\text { - RT + adjuvant temozolomide } \\
\text { - RT + concurrent and adjuvant } \\
\text { temozolomide } \\
\text { - Temozolomidec,4,12,13 } \\
\text { - Lomustine or carmustine } \\
\text { - PCV'4 } \\
\text { - Platinum-based regimens } \\
\text { d,15-17 }\end{array}$ & - None \\
\hline
\end{tabular}

\footnotetext{
aThere are multiple reasonable options, but there is no uniform standard of care at this time for recurrent disease. bIn rare circumstances, treating a patient with chemotherapy without RT may be considered.

cFor patients not previously treated.

dPlatinum-based regimens include cisplatin or carboplatin
}

Full treatment recommendations can be found on ASTR-2 (page 1539); systemic therapy recommendations can be found on BRAIN-D 1 of 15 (above).

\section{Recurrence}

At the time of recurrence, surgery is recommended if resectable disease is present. Because recurrence on neuroimaging may be confounded by treatment effects, biopsy of unresectable disease should be considered to confirm recurrence. There is a propensity for low-grade gliomas to transform to higher-grade gliomas over time. Therefore, documenting the histopathological transformation of a low-grade glioma to a high-grade glioma may also enable patients to have clinical trial opportunities, since most clinical trials in the recurrent setting are for patients with high-grade gliomas. Moreover, sampling of tumor tissue to confirm recurrence is encouraged to obtain tissue for next-generation sequencing, the results of which may inform treatment selection and/or clinical trial eligibility.

Surgery for recurrent disease may be followed by the following treatment options for patients previously treated with fractionated EBRT: (1) chemotherapy; (2) consideration of reirradiation with or without chemotherapy; and (3) palliative/best supportive care. Reirradiation is a good choice if the new lesion is outside the target of previous RT or if the recurrence is small and geometrically favorable. For patients with low-risk features for whom GTR was achieved, observation with no further treatment may be considered.

Based on the strength of the RTOG 9802 results, ${ }^{98,99}$ RT with chemotherapy is a treatment option for patients with recurrent or progressive low-grade gliomas who have not had prior RT. Options include RT + adjuvant PCV, RT + adjuvant TMZ, and RT + concurrent and adjuvant TMZ. RT alone is generally not the preferred treatment option except in select cases, such as a patient with a poor PS, or who does not want to undergo chemotherapy treatment. Chemotherapy alone (eg, TMZ, PCV, carmustine/lomustine) is also a treatment option for these patients, though this is a category 2B option based on less panel consensus. 


\section{Central Nervous System Cancers}

PRINCIPLES OF BRAIN AND SPINAL CORD TUMOR SYSTEMIC THERAPY

\begin{tabular}{|c|c|c|c|}
\hline \multicolumn{4}{|c|}{ ANAPLASTIC GLIOMAS } \\
\hline & \begin{tabular}{|l|} 
Preferred Regimens \\
\end{tabular} & Other Recommended Regimens & Useful in Certain Circumstances \\
\hline $\begin{array}{l}\text { Adjuvant Treatment } \\
\text { Anaplastic oligodendroglioma (1p19q } \\
\text { co-deleted) (KPS } \geq 60 \text { ) }\end{array}$ & $\begin{array}{l}\text { RT with adjuvant PCV } \\
\text { (category 1) } \\
\cdot \text { RT with neoadjuvant PCV } \\
\text { (category 1) }^{\mathrm{e}, 19}\end{array}$ & $\begin{array}{l}\text { - RT with concurrent and adjuvant } \\
\text { TMZ } \\
\text { - RT with adjuvant } T M Z^{21,22}\end{array}$ & • None \\
\hline $\begin{array}{l}\text { Adjuvant Treatment } \\
\text { Anaplastic astrocytoma/anaplastic } \\
\text { oligoastrocytoma, NOSf (KPS } \geq 60 \text { ) }\end{array}$ & $\begin{array}{l}\text { - RT with concurrent and } \\
\text { adjuvant } \mathrm{TMZ}^{23,24} \\
\text { - RT followed by adjuvant TMZ } \\
\text { (12 cycles })^{24}\end{array}$ & $\begin{array}{l}- \text { RT with adjuvant } P C C^{e, 25,26} \\
\cdot \text { RT with neoadjuvant } P C V \text { e }\end{array}$ & - None \\
\hline $\begin{array}{l}\text { Adjuvant Treatment } \\
\text { Anaplastic gliomas (KPS <60) }\end{array}$ & - None & - TMZ9 (category 2B) ${ }^{27}$ & - None \\
\hline Recurrence Therapyh & $\begin{array}{l}\text { - } \text { TMZZ }^{12,13,28,29} \\
\cdot \text { Lomustine or carmustine }^{30} \\
\cdot \text { PCV }^{31} \\
\text { - Bevacizumabi, 32-34 }\end{array}$ & $\begin{array}{l}\text { - Chemotherapyj }+ \text { bevacizumab } \\
\text { - Carmustine or lomustine }+ \\
\text { bevacizumab } \\
\text {, TMZ + bevacizumab } \\
\text { T6 }\end{array}$ & $\begin{array}{l}\text { - If failure or intolerance to the } \\
\text { preferred or other recommended } \\
\text { regimens } \\
\text { - Etoposide } \\
\text { P Platinum-based regimens } \text { (category }^{\mathrm{d}, 39-41} \\
\text { (category 3) }\end{array}$ \\
\hline
\end{tabular}

dPlatinum-based regimens include cisplatin or carboplatin.

eThe panel recommends that PCV be administered after RT (as per EORTC 26951) since the intensive PCV regimen given prior to RT (RTOG 9402) was not tolerated as well.

${ }^{\mathrm{f}}$ The WHO 2016 classification of CNS tumors has deleted oligoastrocytoma as a diagnostic category; however, oligoastrocytoma NOS and anaplastic oligoastrocytoma NOS may continue to be used for tumors that cannot be classified as either astrocytoma or oligodendroglioma due to the absence of appropriate molecular testing. gConsider TMZ if tumor is MGMT promoter methylated.

hStrongly suggest consideration of clinical trials prior to treating recurrent disease with standard chemotherapy, as additional therapies may eliminate the majority of clinical trial options.

iPatients who have evidence of radiographic progression may benefit from continuation of bevacizumab to prevent rapid neurologic deterioration

${ }^{j}$ Bevacizumab + chemotherapy can be considered if bevacizumab monotherapy fails and it is desirable to continue the steroid-sparing effects of bevacizumab

Full treatment recommendations for recurrent or progressive low-grade gliomas can be found on ASTR-3 (page 1540).

\section{Anaplastic Gliomas and Glioblastomas}

High-grade gliomas (defined as WHO grade III and IV gliomas) are the most common type of brain cancer, accounting for more than half of all malignant primary tumors of the CNS. ${ }^{78}$ Whereas the prognosis for glioblastoma (grade IV glioma) is grim (5-year survival rates between $1 \%-19 \%$, depending on age), outcomes for anaplastic gliomas (grade III gliomas) are typically better, depending on the molecular features of the tumor. ${ }^{54}$ Challenges regarding treatment of glioblastoma include the inability of most systemic therapy agents to penetrate the blood-brain barrier (BBB) and heterogeneity among genetic drivers. ${ }^{121}$

High-grade astrocytomas diffusely infiltrate surrounding tissues and frequently cross the midline to involve the contralateral brain. Patients with these neoplasms often present with symptoms of increased intracranial pressure, seizures, or focal neurologic findings related to the size and location of the tumor and associated vasogenic edema. High-grade astrocytomas usually do not have associated hemorrhage or calcification but can produce considerable edema and mass effect, and they enhance after the administration of intravenous contrast. Tumor cells have been found in peritumoral edema, which corresponds to the T2-weighted MRI abnormalities. Thus, this volume is frequently used to define RT treatment volumes.

It can be challenging to assess the results of therapy by MRI, because the extent and distribution of contrast enhancement, edema, and mass effect are a function of BBB integrity. Thus, factors that increase permeability of the BBB (such as surgery, RT, tapering of corticosteroids, and immunotherapies) can mimic tumor progression radiographically by increasing the presence of contrast enhancement and associated vasogenic edema. Furthermore, anti-VEGF therapy (ie, bevacizumab) suppresses vascular permeability and provides a radiographic appearance of a response, despite residual disease (pseudoresponse). ${ }^{122}$

Anaplastic oligodendrogliomas are relatively rare. ${ }^{78}$ Although these tumors can be confused with glioblastoma 
Central Nervous System Cancers

PRINCIPLES OF BRAIN AND SPINAL CORD TUMOR SYSTEMIC THERAPY

\begin{tabular}{|c|c|c|c|}
\hline \multicolumn{4}{|c|}{ GLIOBLASTOMA } \\
\hline & Preferred Regimens & Other Recommended Regimens & Useful in Certain Circumstances \\
\hline Adjuvant Treatment & $\begin{array}{l}\cdot \text { RT with concurrent and adjuvant } \mathrm{TMZ}^{42,43} \\
\pm \mathrm{TTF}\end{array}$ & & $\begin{array}{l}\cdot \text { RT with concurrent and adjuvant TMZ (for } \\
\text { patients age } 70 \text { or younger and KPS }<60)^{45} \\
\text { - TMZ (for patients with MGMT promoter- } \\
\text { methylated tumors and KPS }<60 \text { or age }>70 \\
\text { years and KPS } \geq 60)^{42,46} \\
\text { - RT with concurrent and adjuvant lomustine } \\
\text { and TMZ (for patients with MGMT promoter- } \\
\text { methylated tumors, KPS } \geq 60 \text {, and age } \leq 70 \\
\text { years) (category 2B) }\end{array}$ \\
\hline $\begin{array}{l}\text { Recurrence } \\
\text { Therapyh,m }\end{array}$ & $\begin{array}{l}\text { - Bevacizumab }{ }^{\mathrm{i}, \mathrm{I}, 48-51} \\
\text { - Temozolomide }{ }^{13,29,52,53} \\
\text { - Lomustine or carmustine } \\
\text { - PCV } 58,59 \\
\text { - Regorafenib }\end{array}$ & $\begin{array}{l}\text { - Chemotherapy }{ }^{j}+\text { bevacizumab } \\
\text { - Carmustine or lomustine + } \\
\text { bevacizumab }^{61,62} \\
\text { - TMZ + bevacizumab } \\
\text { 63,64 }\end{array}$ & $\begin{array}{l}\text { - If failure or intolerance to the preferred or other } \\
\text { recommended regimens } \\
\text { Etoposide (category 2B) } \\
\left.\text { - Platinum-based regimens }{ }^{\text {d }} \text { (category } 3\right)^{65,66}\end{array}$ \\
\hline
\end{tabular}

dPlatinum-based regimens include cisplatin or carboplatin.

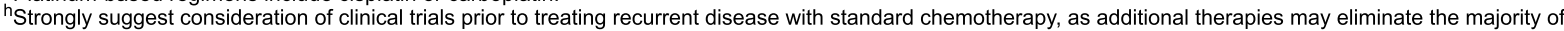
clinical trial options

'Patients who have evidence of radiographic progression may benefit from continuation of bevacizumab to prevent rapid neurologic deterioration.

jBevacizumab + chemotherapy can be considered if bevacizumab monotherapy fails and it is desirable to continue the steroid-sparing effects of bevacizumab.

${ }^{k}$ Moderate to significant myelosuppression was observed, but the toxicity profile for this regimen is not yet fully defined.

IAn FDA-approved biosimilar is an appropriate substitute for bevacizumab.

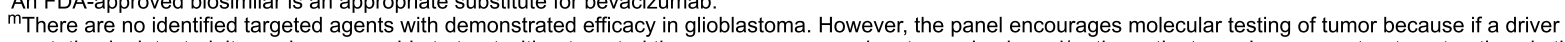
mutation is detected, it may be reasonable to treat with a targeted therapy on a compassionate use basis and/or the patient may have more treatment options in the context of a clinical trial. Molecular testing also has a valuable role in improving diagnostic accuracy and prognostic stratification that may inform treatment selection.

histopathologically, if molecular analysis detects that the tumor is 1p19q codeleted and IDH1-mut or IDH2-mut, then the tumor is considered to be an anaplastic oligodendroglioma. ${ }^{1}$ This distinct subtype has a much better prognosis compared with other high-grade gliomas (anaplastic astrocytomas and glioblastomas).

\section{Treatment Overview}

\section{Surgery}

The goals of surgery are to obtain a diagnosis, alleviate symptoms related to increased intracranial pressure or compression by tumor, increase survival, and decrease the need for corticosteroids. A meta-analysis including six studies with 1618 patients with glioblastoma showed that GTR is associated with superior OS and PFS, compared with incomplete resection and biopsy. ${ }^{123}$ Unfortunately, the infiltrative nature of high-grade astrocytomas frequently renders GTR difficult. There are data suggesting that resection of all fluid-attenuated inversion recovery (FLAIR) signal abnormality in high-grade $I D H$-mut gliomas is associated with improved survival. ${ }^{124}$ However, a newer and larger study did not find greater benefit of resection in $\mathrm{IDH}$ mut tumors compared with $I D H$-wt high-grade gliomas. ${ }^{125}$

Unfortunately, nearly all high-grade gliomas recur. Reresection at the time of recurrence may improve the outcome for select patients. ${ }^{126}$ According to an analysis by Park et al, ${ }^{127}$ tumor involvement in specific critical brain areas, poor KPS score, and large tumor volume $\left(\geq 50 \mathrm{~cm}^{3}\right)$ were associated with unfavorable reresection outcomes.

\section{Radiation Therapy}

Conformal RT (CRT) techniques, which include 3-dimensional CRT (3D-CRT) and IMRT are recommended for performing focal brain irradiation. IMRT often will provide superior dosimetric target coverage and better sparing of critical structures than 3D-CRT. ${ }^{103}$ Several randomized controlled trials conducted in the 1970s showed that radiation improved both local control and survival in patients with newly diagnosed high-grade gliomas. ${ }^{128,129}$ Sufficient radiation doses are required to maximize this survival benefit. However, radiation dose escalation alone above 60 Gy has not been shown to be 
beneficial. ${ }^{130}$ The recommended radiation dose for highgrade astrocytomas is $60 \mathrm{~Gy}$ in $2.0 \mathrm{~Gy}$ fractions or $59.4 \mathrm{~Gy}$ in $1.8 \mathrm{~Gy}$ fractions with an initial RT plan to $46 \mathrm{~Gy}$ in $2 \mathrm{~Gy}$ fractions or 45 to 50.4 Gy in 1.8 fractions, respectively, followed by a boost plan of $14 \mathrm{~Gy}$ in $2 \mathrm{~Gy}$ fractions or 9 to 14.4 Gy in 1.8 Gy fractions, respectively. ${ }^{130}$

Anaplastic oligodendrogliomas are conventionally treated with the same dose of radiation as high-grade astrocytomas; however, given the better prognosis in patients with anaplastic oligodendroglioma, radiation treatments are generally administered in a lower dose per fraction (1.8 Gy/fraction vs $2.0 \mathrm{~Gy} /$ fraction) to theoretically decrease the risk of late side effects. Accordingly, as per trials such as RTOG $9813,{ }^{51}$ these gliomas are treated to $50.4 \mathrm{~Gy}$ in $1.8 \mathrm{~Gy}$ fractions for 28 fractions followed by a five-fraction boost of $1.8 \mathrm{~Gy} /$ fraction to a total of $59.4 \mathrm{~Gy}$. RT targets for high-grade gliomas are generated from a gross tumor volume (GTV), CTV, and planning target volume (PTV). The GTV encompasses any gross tumor remaining after maximal safe resection as well as the surgical cavity as determined by postoperative imaging. Strategies for GTV definition vary with respect to the inclusion of edema in an initial target volume. When edema is included in an initial phase of treatment, fields are usually reduced for the last phase of treatment. The CTV is an expansion of the GTV by adding an approximately $2-\mathrm{cm}$ margin for grade III and IV gliomas (although smaller CTV expansions are supported in the literature and can be appropriate) to account for a nonenhancing tumor. The CTV is then expanded to a PTV to account for daily setup errors and image registration. The boost target volume will typically encompass only the gross residual tumor and the resection cavity.

Special attention has been given to determining the optimal therapy in older adults with glioblastoma, given their especially poor prognosis, often limited functional status, and increased risk of developing side effects. Overall, the approach in these patients has been to reduce treatment time while maintaining treatment efficacy. Roa et al randomized patients 60 years or older with a poor PS (KPS < 70) to 60 Gy in 30 fractions given over 6 weeks versus $40 \mathrm{~Gy}$ in 15 fractions given over 3 weeks and found no difference in survival between these two regimens. ${ }^{131}$ However, fewer patients who received $40 \mathrm{~Gy}$ over a shorter time period required a posttreatment increase in corticosteroid dose, compared with the patients who received 60 Gy over the longer time period ( $23 \%$ vs $49 \%$, respectively; $P=.02$ ). A subsequent study also supports using a regimen of $34 \mathrm{~Gy}$ in 10 fractions over 2 weeks in older adult patients. ${ }^{37}$ Moreover, another study performed by Roa et al showed that an even shorter course of focal brain radiation consisting of $25 \mathrm{~Gy}$ in 5 fractions over 1 week is a reasonable alternative to $40 \mathrm{~Gy}$ in 15 fractions over 3 weeks in patients with newly diagnosed glioblastoma who have a poor prognosis (ie, patients who are older adults and/or frail). ${ }^{132}$ However, this was a small study that had some limitations, notably overly broad eligibility criteria and poorly defined noninferiority margin. ${ }^{133,134}$

A randomized trial of hypofractionated RT (40 Gy given over 3 weeks) with concurrent and adjuvant TMZ versus hypofractionated RT alone in patients 65 years and older showed an improvement in median OS and PFS with the addition of concurrent and adjuvant TMZ (5-year OS of $9.8 \%$ vs $1.9 \%$, respectively; median OS of 14.6 months vs 12.1 months, respectively; HR for mortality, 0.63 , 95\% CI, $0.53-0.75, P<.001 ; 5$-year PFS of $4.1 \%$ vs $1.3 \%$, respectively; $\mathrm{HR}, 0.56$; $95 \% \mathrm{CI}, 0.47-0.66$; $P<.001) .{ }^{135}$ The largest benefit was noted in patients with MGMT promoter methylation (see "Systemic Therapy for Glioblastoma," page 1554). Of note, a comparison of standard focal brain radiation (60 Gy given over 6 weeks) with concurrent and adjuvant TMZ versus hypofractionated radiation (40 Gy given over 3 weeks) with concurrent and adjuvant TMZ in elderly patients has not been performed in patients 65 years and older. Therefore, standard radiation (60 Gy given over 6 weeks) with concurrent and adjuvant TMZ (with or without alternating electric field therapy; see discussion of this treatment option in subsequent sections) is also a reasonable treatment option for an older adult patient who has a good PS and wishes to be treated aggressively. Ultimately, quality of life remains an important consideration in the optimal management of this patient population.

\section{Systemic Therapy}

\section{Anaplastic Oligodendroglioma}

The addition of PCV to RT for the treatment of newly diagnosed anaplastic oligodendrogliomas is supported by results from two phase III trials, one which tested RT followed by PCV for 6 cycles (EORTC 26951 ${ }^{136,137}$ ) and the other which assessed 4 cycles of dose-intensive PCV administered prior to RT (RTOG 9402 $27,138,139$ ). Both studies compared the combination therapy to RT alone and found significant increases in median OS when PCV was added to RT for the upfront management of $1 \mathrm{p} 19 \mathrm{q}$ codeleted tumors.

The EORTC 26951 trial showed that, among the entire group of 368 histopathologically diagnosed study patients with anaplastic oligodendroglioma or anaplastic oligoastrocytoma, RT followed by 6 cycles of PCV significantly improved median PFS and OS (42.3 vs 30.6 months; HR, 0.75; 95\% CI, 0.60-0.95; $P=.018$ ) compared with RT alone. ${ }^{137}$ Moreover, in an exploratory subgroup analysis of the 80 patients whose tumors were $1 p 19 q$ codeleted, the benefit was even more pronounced (OS not reached in the RT + PCV group vs 112 months in the RT group; HR, 0.56; 95\% CI, 0.31-1.03). ${ }^{14,136,137}$ 
RTOG 9402 randomized 291 patients with histopathologically diagnosed anaplastic oligodendroglioma or anaplastic oligoastrocytoma to treatment with an intensive PCV regimen followed by RT or RT alone. ${ }^{139}$ In contrast to the EORTC 26951 study, no difference in median OS was observed between the two arms (4.6 years vs 4.7 years; $\mathrm{HR}, 0.79 ; 95 \% \mathrm{CI}, 0.60-1.04 ; P=.10$ ). However, an unplanned subgroup analysis of the 126 patients whose tumors werelp19q codeleted found a doubling in median OS (14.7 vs 7.3 years; HR, $0.59 ; 95 \%$ CI, $0.37-0.95 ; P=.03$ ) when PCV was added to RT as upfront treatment.

As would be predicted, in both studies toxicity was higher in the treatment arms that included PCV. In EORTC 26951, $70 \%$ of patients in the RT followed by PCV arm did not complete the planned 6 cycles of treatment. ${ }^{136,137}$ In RTOG 9402, there was also a high rate of study treatment discontinuation and acute toxicities (mainly hematologic), including 2 early deaths attributed to PCV-induced neutropenia. ${ }^{138,139}$ Given the similar efficacy results of the two studies, and the two deaths that occurred from the intensive PCV regimen in RTOG 9402, PCV administered after RT is optimal, as per EORTC 26951.

The phase III CODEL study was designed to assess the efficacy of TMZ for the treatment of newly diagnosed anaplastic oligodendrogliomas. The initial treatment arms were RT alone, RT + TMZ, and TMZ alone. Initial results showed that patients who received TMZ alone had significantly shorter PFS than patients treated with RT (either RT alone or with TMZ) (2.9 years vs not reached, respectively; $\mathrm{HR}, 3.12$; 95\% CI, 1.26-7.69; $P=.009) .{ }^{140}$ When the results of RTOG 9402 and EORTC 26951 were reported showing significant improvement in median OS with RT + PCV upfront, the CODEL study was redesigned to compare RT + PCV to RT + TMZ in patients with anaplastic oligodendroglioma as well as lowgrade oligodendroglioma. This study is ongoing.

\section{Anaplastic Astrocytoma}

The RTOG 9813 trial showed that RT with concurrent TMZ resulted in similar outcomes as RT with concurrent nitrosourea (either CCNU [lomustine] or BCNU [carmustine]) therapy in patients with newly diagnosed anaplastic astrocytomas, with perhaps slightly better PFS with TMZ (HR, 0.70; 95\% CI, 0.50-0.98; $P=.039$ ). ${ }^{51}$ However, the toxicity of nitrosourea was significantly worse than for TMZ, and resulted in higher rates of discontinuation due to toxicity ( $79 \%$ vs $40 \%$, respectively; $P<.001$ ). The ongoing CATNON phase 3 randomized trial is testing RT alone, as well as RT with adjuvant TMZ, concurrent TMZ, or both, in patients with newly diagnosed anaplastic astrocytoma. An initial interim analysis showed adjuvant TMZ significantly improved
PFS (HR, 0.62; 95\% CI, 0.50-0.76) and OS (HR, 0.67; 95\% CI, 0.51-0.88). ${ }^{117}$ Median OS for the group of patients treated with post-RT TMZ had not been reached, but median OS at 5 years was $55.9 \%$ (95\% CI, 47.2-63.8) with and $44.1 \%$ (36.3-51.6) without adjuvant TMZ. A second interim analysis showed that patients with $I D H$-mut anaplastic astrocytomas benefit from treatment with adjuvant TMZ (HR, 0.41; 95\% CI, 0.27-0.64), but not those with tumors that are $I D H$-wt (HR, 1.05; 95\% CI, 0.73-1.52). ${ }^{141}$ There was also no definite benefit to concurrent TMZ in patients with $I D H$-mut anaplastic astrocytomas (HR, 0.71; 95\% CI, 0.35-1.42; $P=.32$ ). However, the findings from the second interim analysis are currently available in abstract form only. Further follow-up and molecular analyses are ongoing.

\section{Glioblastoma}

Adjuvant involved-field RT with concurrent and adjuvant TMZ is the standard recommended treatment of patients with newly diagnosed glioblastoma and good PS based on the results of the phase III, randomized EORTC-NCIC study of 573 patients with newly diagnosed glioblastoma who were age $\leq 70$ years and had a WHO PS $\leq 2 .{ }^{135}$ Patients received either 1) daily TMZ administered concomitantly with postoperative RT followed by 6 cycles of adjuvant TMZ; or 2) RT alone. The chemoradiation arm resulted in a statistically better median survival (14.6 vs 12.1 months) and 2-year survival (26.5\% vs $10.4 \%)$ when compared with RT alone. Final analysis confirmed the survival advantage at 5 years $(10 \%$ vs $2 \%) .{ }^{135}$ However, the study design does not shed light on which component is responsible for the improvement: TMZ administered with RT, TMZ following RT, or possibly both.

The TMZ dose used in the EORTC-NCIC trial is $75 \mathrm{mg} / \mathrm{m}^{2}$ daily concurrent with $\mathrm{RT}$, then 150 to $200 \mathrm{mg} / \mathrm{m}^{2}$ postirradiation on a 5 -day schedule every 28 days. Alternate schedules, such as a $75-100 \mathrm{mg} / \mathrm{m}^{2}$ for 21 out of 28 days regimen or $50 \mathrm{mg} / \mathrm{m}^{2}$ daily, have been explored in a phase II trial for newly diagnosed glioblastoma. ${ }^{142}$ However, a comparison of the doseintense $21 / 28$ and standard 5/28 schedules in the RTOG 0525 phase III study showed no difference in PFS, OS, or by MGMT methylation status with the postradiation dose-intense TMZ, compared with the standard postradiation TMZ dose. ${ }^{143}$ A pooled analysis of individual patient data from 4 randomized trials ${ }^{119,143-145}$ of patients with newly diagnosed glioblastoma determined that treating with postradiation TMZ beyond 6 cycles does not improve OS, even for patients whose tumors are MGMT promoter methylated. ${ }^{146} \mathrm{~A}$ recent prospective, randomized phase II study showed no improvement in 6-month PFS, PFS, or OS with continuing treatment with TMZ beyond 6 cycles, and doing so was associated with greater toxicity. ${ }^{147}$ 


\section{MGMT Promoter Methylated Glioblastoma}

The presence of MGMT promoter methylation in glioblastoma is both a prognostic marker and a predictive one for response to treatment with alkylating agents. In the small $(n=31)$, single arm phase II UKT-03 trial, ${ }^{148,149}$ postoperative RT and TMZ combined with lomustine in patients with newly diagnosed glioblastoma resulted in a median OS of 34.3 months, ${ }^{148}$ which compared favorably to the historical control data of 23.4 months in patients with MGMT promoter methylated tumors who were treated with RT and TMZ in the EORTC-NCIC trial. ${ }^{135}$ Based on this improvement in survival with combination alkylating agents in patients with MGMT promoter methylated glioblastoma, the phase III CeTeG/NOA-09 trial randomized patients with newly diagnosed MGMT promoter methylated glioblastoma (age 18-70 and KPS $\geq$ 70) to treatment with RT and lomustine $+\mathrm{TMZ}$ or RT and TMZ alone. ${ }^{150}$ Analysis of the modified intent-to-treat population $(n=129)$ showed that OS was significantly improved in the TMZ + lomustine arm vs the TMZ arm (median OS of 48.1 months vs 31.4 months, respectively; $P=.049$ ). Of note, PFS was not significantly improved, which the investigators hypothesized could have been due to a higher incidence of pseudoprogression in the $\mathrm{TMZ}+$ lomustine arm. Grade 3 and 4 adverse events were only slightly higher in the TMZ+lomustine arm (59\% vs $51 \%$, respectively), but the study was too small to adequately define the toxicity profile of RT with $\mathrm{TMZ}+$ lomustine. Analysis of health-related quality of life showed no significant differences between the study arms. ${ }^{151}$

\section{Older Adults}

Building on the findings that hypofractionated RT alone has similar efficacy and is better tolerated compared with standard RT alone in older adults with newly diagnosed glioblastoma, a phase III randomized trial with 562 newly diagnosed patients 65 years of age or older compared hypofractionated RT with concurrent and adjuvant TMZ to hypofractionated radiation alone. Patients in the combination therapy arm had better PFS (5.3 months vs 3.9 months; HR, 0.50; 95\% CI, $0.41-0.60 ; P<.001)$ and median OS (9.3 months vs 7.6 months; HR, 0.67; 95\% CI, 0.56-0.80; $P<.001)$ compared with patients treated with hypofractionated RT alone. ${ }^{118}$ The greatest improvement in median OS was seen in patients with MGMT promoter methylated tumors (13.5 months RT + TMZ vs 7.7 months RT alone; HR, 0.53; 95\% CI, 0.38-0.73; $P<.001$ ). The benefit of adding TMZ to RT was smaller in patients with MGMT promoter unmethylated tumors and did not quite reach statistical significance (10.0 months vs 7.9 months, respectively; HR, 0.75; 95\% CI, 0.56-1.01; $P=.055 ; P=.08$ for interaction).
Two phase III studies in elderly newly diagnosed glioblastoma patients assessed treatment with TMZ alone versus radiation. ${ }^{37,38}$ The Nordic trial randomized 291 patients aged 60 years and older with good PS across 3 treatment groups: TMZ, hypofractionated RT, or standard RT. ${ }^{37}$ Patients older than 70 years had better survival with TMZ or hypofractionated RT compared with standard RT, and patients whose tumors were MGMT promoter methylated benefitted more from treatment with TMZ compared with patients with MGMT promoter unmethylated tumors (median OS 9.7 vs 6.8 months; HR, 0.56; 95\% CI, 0.34-0.93; $P=.02$ ). The NOA-08 study assessed the efficacy of TMZ alone compared with standard RT in 373 patients aged 65 years and older. ${ }^{38}$ TMZ was found to be noninferior to standard RT; median OS was similar in both groups (8.6 months in the TMZ arm vs 9.6 months in the standard RT arm; HR, 1.09; 95\% CI, 0.84-1.42; $P$ (noninferiority) $=0.033$ ). For patients whose tumors were MGMT promoter methylated, eventfree survival was longer with TMZ treatment compared with standard RT (8.4 months vs 4.6 months). Neither the Nordic trial nor the NOA-08 trial included a combination RT and TMZ control arm, which is the treatment regimen typically offered to patients who are fit enough to tolerate it, regardless of age. Although radiation in combination with TMZ is recommended over single-modality therapy for newly diagnosed patients with glioblastoma who are older than 70 years of age and have good PS, the results of these two phase III studies support the recommendation that TMZ alone as initial therapy may be a reasonable option for those elderly patients who have MGMT promoter methylated tumors and would initially prefer to delay treatment with radiation. ${ }^{37,38}$

\section{Alternating Electric Field Therapy}

In 2015, the FDA approved alternating electric field therapy for the treatment of patients with newly diagnosed glioblastoma based on the results of the openlabel phase III EF-14 clinical trial. This portable medical device generates low-intensity alternating electric fields to stop mitosis/cell division. In the EF-14 trial, 695 patients with newly diagnosed glioblastoma and good PS (KPS $\geq 70$ ) were randomized to $\mathrm{TMZ}$ alone on a 5/28-day schedule or the same TMZ and alternating electric field therapy, following completion of standard focal brain radiation and daily TMZ. ${ }^{152}$ The results of the study showed an improvement in median PFS (6.7 vs 4.0 months, respectively; HR, 0.63; 95\% CI, 0.52-0.76; $P<.001)$ and OS (20.9 vs 16.0 months, respectively; HR, 0.63 ; 95\% CI, 0.53-0.76; $P<.001$ ) in patients who received TMZ plus alternating electric field therapy. ${ }^{153}$ The number of adverse events was not statistically different between the two treatment groups except for a greater frequency of mild to moderate local skin irritation/itchiness in the 
patients treated with the alternating electric fields. ${ }^{154}$ There was no increased frequency of seizures. ${ }^{155,156}$ Based on the results of this study, concurrent treatment with adjuvant TMZ and alternating electric fields is a category 1 recommendation for newly diagnosed glioblastoma patients 70 years of age or younger who have a good PS. This is also considered a reasonable treatment option for patients older than 70 years of age with good PS and newly diagnosed glioblastoma who are treated with standard focal brain radiation and concurrent daily TMZ.

\section{Therapy for Recurrence}

Patients with malignant gliomas eventually develop tumor recurrence or progression. Surgical resection of locally recurrent disease is reasonable followed by treatment with chemotherapy. Unfortunately, there is no established second-line therapy for recurrent gliomas. If there has been a long time interval between stopping TMZ and development of tumor progression, it is reasonable to restart a patient on TMZ, ${ }^{157}$ particularly if the patient's tumor is MGMT methylated. Similarly, a nitrosourea, such as carmustine or lomustine, ${ }^{158-161}$ would be a reasonable second-line therapy, especially in a patient whose tumor is MGMT methylated. Although no studies of bevacizumab in patients with recurrent glioblastoma have demonstrated an improvement in survival, bevacizumab is FDA approved for the treatment of recurrent glioblastoma based on improvement in PFS. ${ }^{162-164}$ Of note, improvement in PFS may be due to bevacizumab's ability to decrease BBB permeability (resulting in less contrast enhancement and vasogenic edema) rather than a true antitumor effect. ${ }^{165,166}$ Treatment with regorafenib for recurrent glioblastoma is supported by the results of a randomized phase II trial in which OS was greater for patients randomized to receive regorafenib, compared with those who received lomustine (median OS of 7.4 months vs 5.6 months, respectively; HR, 0.50 ; $95 \%$ CI, $0.33-0.75 ; P<.001){ }^{167}$ Of note, the median OS in the lomustine arm in this trial was lower than reported in other randomized phase II and III trials. A phase III study of regorafenib is being planned.

Other routes of chemotherapy delivery have been evaluated. Local administration of carmustine using a biodegradable polymer (wafer) placed intraoperatively in the surgical cavity has demonstrated a statistically significant improvement in survival for patients with recurrent high-grade gliomas ( 31 vs 23 weeks; adjusted HR, $0.67 ; P=.006) .{ }^{168}$ Patients who receive carmustine wafers are at greater risk for seizures and postoperative infections. When wafers are used, it is important to achieve a watertight dural closure and have sufficient use of steroids and antiepileptics in the perioperative period to prevent adverse events. ${ }^{169}$ Clinicians and patients should be aware that treatment with the carmustine wafer may prevent participation in a clinical trial involving a locally delivered investigational agent.

Alternating electric field therapy is also FDA approved for treating recurrent glioblastoma based on the safety results of this medical device from the EF-11 clinical trial. ${ }^{170}$ This phase III study randomized 237 patients with recurrent glioblastoma to alternating electric field therapy or the treating oncologist's choice of chemotherapy. The study did not meet its primary endpoint of demonstrating an improvement in survival in the cohort of patients treated with alternating electric field therapy. Although median OS was similar in both of the treatment arms (6.6 vs 6 months), the study had not been powered for a noninferiority determination. Due to lack of clear efficacy data for alternating electric field therapy in EF-11, the panel is divided about recommending it for the treatment of recurrent glioblastoma. Similarly, reirradiation may be reasonable to consider for some recurrent glioblastoma patients, but the panel is also divided about this option. A systematic review including 50 noncomparative studies of 2095 patients with recurrent glioblastoma who were treated with reirradiation showed pooled 6- and 12-month OS rates of $73 \%$ and $36 \%$, respectively, and 6 - and 12 -month PFS rates of $43 \%$ and $17 \%$, respectively. ${ }^{171}$ Over half of the studies (29 out of 50) were rated as poor quality, indicating a need for better quality studies in this area. Further, there is no recommended dose or type of radiation used in the recurrent setting due to inconsistent trial design among these studies.

\section{NCCN Recommendations}

\section{Primary Treatment}

When a patient presents with a clinical and radiologic picture suggestive of a high-grade glioma, neurosurgical input is needed regarding the feasibility of maximal safe resection. For first-line treatment of high-grade glioma, the NCCN Guidelines recommend maximal safe resection whenever possible. Use of intraoperative MRI and intraoperative fluorescence-guided surgery with 5-ALA may potentially allow for more complete resection. ${ }^{172,173}$ One exception is when CNS lymphoma is suspected; a biopsy should be performed before steroids are administered, and management should follow the corresponding pathway if the diagnosis is confirmed. When maximal resection is performed, the extent of tumor debulking should be documented with a postoperative MRI scan with and without contrast performed within 48 hours after surgery. Multidisciplinary consultation is encouraged once the pathology is available. See GLIO-1 in the algorithm (page 1541).

\section{Adjuvant Therapy}

RT is generally recommended after maximal safe resection for the treatment of high-grade gliomas to 
improve local control and survival. For postoperative treatment of anaplastic gliomas in patients with good PS (KPS $\geq 60$ ), combination therapy with focal brain radiation combined with PCV or TMZ are among the recommended options. For patients with anaplastic oligodendroglioma, RT plus PCV, given before or after $\mathrm{RT}$, is preferred, based on the results of the RTOG $9402^{27,139}$ and EORTC 26951 studies. $^{136,137}$ The panel advises administering PCV after RT as per EORTC 26951 instead of the dose-intensive PCV used prior to RT in the RTOG 9402 study $^{139}$ due to better patient tolerance. RT, with or without concurrent TMZ, followed by adjuvant TMZ is also a reasonable option, ${ }^{174}$ particularly if it is predicted that the patient might have significant difficulty tolerating PCV due to age or coexisting medical conditions. The panel awaits the results of CODEL to see if treatment with TMZ will be as efficacious as PCV in this patient population.

In the case of patients with anaplastic astrocytoma and anaplastic oligoastrocytoma (NOS) and good PS, RT, with or without concurrent TMZ and followed by adjuvant TMZ is preferred based on the first interim analysis results of the CATNON trial showing improvement in survival with RT followed by 12 cycles of TMZ compared with RT alone. ${ }^{117}$ However, for newly diagnosed anaplastic oligoastrocytoma patients, RT with PCV administered before or afterward is also an acceptable treatment option. ${ }^{175,176}$

For patients with anaplastic gliomas and a poor PS (KPS $<60$ ), treatment options recommended in the NCCN Guidelines are limited to single-modality therapies due to concerns about the ability of these patients to tolerate the toxicity associated with combination regimens. Patients with a poor PS can be managed by RT (hypofractionation is preferred over standard fractionation), TMZ alone (considered for patients whose tumors are MGMT promoter methylated but is a category $2 \mathrm{~B}$ option), or palliative/best supportive care.

Full treatment recommendations for anaplastic gliomas can be found on GLIO-2 (page 1542); systemic therapy recommendations can be found on BRAIN-D 2 of 15 (page 1553).

For patients diagnosed with glioblastoma, the adjuvant options mainly depend on the patient's age, PS (as defined by KPS), and MGMT promoter methylation status (see GLIO-3 and GLIO-4 on pages 1543 and 1544). ${ }^{34,37,135,177}$ Category 1 recommendations for patients aged 70 years and younger with a good PS, regardless of the tumor's MGMT methylation status, include standard brain RT plus concurrent and adjuvant TMZ with or without alternating electric field therapy. Because patients with newly diagnosed MGMT promoter unmethylated glioblastoma are likely to receive less benefit from TMZ, RT alone is included as a reasonable option, particularly if the patient is eligible to participate in a clinical trial, which omits the use of upfront TMZ.

Category 1 treatment recommendations for patients older than 70 years of age with newly diagnosed glioblastoma, a good PS, and MGMT promoter methylated tumors include hypofractionated brain RT plus concurrent and adjuvant $\mathrm{TMZ}^{118}$ or standard brain RT plus concurrent and adjuvant TMZ and alternating electric field therapy. For those patients older than 70 years with newly diagnosed glioblastoma, a good PS, and with MGMT unmethylated or indeterminant tumors, hypofractionated brain radiation with concurrent and adjuvant $\mathrm{TMZ}^{118}$ is preferred, but standard brain RT plus concurrent and adjuvant TMZ and alternating electric field therapy is also a reasonable option (category 1$)^{152,153}$ for those elderly patients who want to be treated as aggressively as possible. The complete list of recommendations that the panel did not consider category 1 can be found in the treatment algorithms for patients with glioblastoma who are older than 70 years.

Treatment recommendations for patients with newly diagnosed glioblastoma and KPS below 60 (regardless of age) include hypofractionated brain RT possibly with concurrent and adjuvant TMZ for patients aged 70 years or younger, TMZ alone (for patients with MGMT promoter methylated tumors), or palliative/best supportive care.

\section{Follow-up and Recurrence}

Patients should be followed closely with serial brain MRI scans (at 2-8 weeks postirradiation, then every 2-4 months for 3 years, then every 3-6 months indefinitely) after the completion of treatment of newly diagnosed disease. Scans may appear worse during the first 3 months or longer after completion of RT even though there may be no actual tumor progression. ${ }^{121}$ This finding of "pseudoprogression" occurs more often in patients whose tumors are MGMT promoter methylated. ${ }^{178,179}$ Early MRI scans allow for appropriate titration of corticosteroid doses based on the extent of mass effect and brain edema. Later scans are used to identify tumor recurrence. Early detection of recurrence is warranted, because local and systemic treatment options are available for patients with recurrent disease. Biopsy, MR spectroscopy, MR perfusion, or brain PET/CT can be considered to try to determine if the changes seen on brain MRI are due to pseudoprogression or RT-induced necrosis versus actual disease progression. ${ }^{180,181}$ RT-induced necrosis tends to be detected between 6 and 24 months following RT treatment. ${ }^{179}$

Management of recurrent tumors depends on the extent of disease and patient condition (see GLIO-5 on page 1545). The efficacy of current treatment options for recurrent disease remains poor; therefore, enrollment in a clinical trial, whenever possible, is preferred for the 
management of recurrent disease. Preferred chemotherapy options for recurrent disease include retreatment with TMZ (if there has been a long interval between completion of adjuvant TMZ and development of recurrent disease), ${ }^{11,157,182-184}$ carmustine/lomustine, ${ }^{158-161,185}$ bevacizumab, ${ }^{162,186-191}$ regorafenib, ${ }^{167}$ and $\mathrm{PCV}^{112,192,193}$ (see BRAIN-D 3 of 15 for the list of systemic therapy recommendations, page 1554). A patient with a poor PS should receive palliative/best supportive care.

\section{Brain Metastases}

Metastases to the brain are the most common intracranial tumors in adults and may occur up to 10 times more frequently than primary brain tumors. Populationbased data reported that about $8 \%-10 \%$ of patients with cancer are affected by symptomatic metastatic tumors in the brain. ${ }^{194,195}$ Based on autopsy studies, brain metastases have been shown to be present in $25 \%$ of patients with cancer. ${ }^{196}$

As a result of advances in diagnosis and treatment, many patients improve with proper management and do not die of progression of these metastatic lesions. Primary lung cancers are the most common source, ${ }^{197}$ although melanoma has a high predilection to spread to the brain. ${ }^{198}$ Diagnosis of CNS involvement is increasing in patients with breast cancer as therapy for metastatic disease is improving. ${ }^{199}$

Nearly $80 \%$ of brain metastases occur in the cerebral hemispheres, an additional $15 \%$ occur in the cerebellum, and 5\% occur in the brainstem. ${ }^{200}$ These lesions typically follow a pattern of hematogenous spread to the graywhite junction where the relatively narrow caliber of the blood vessels tends to trap tumor emboli. The majority of cases have multiple brain metastases evident on MRI scans. The presenting signs and symptoms of metastatic brain lesions are similar to those of other mass lesions in the brain, such as headache, seizures, and neurologic impairment.

\section{Treatment Overview}

\section{Surgery}

Despite advances in surgical technique, surgery alone for brain metastases is not sufficient for achieving local control. ${ }^{201,202}$ The objectives of surgery for brain metastasis include retrieval of tissue for diagnosis, reduction of mass effect, and improvement of edema. ${ }^{203}$ To promote local control following resection of a brain metastasis, adjuvant RT represents an acceptable treatment strategy, discussed further below. Randomized trials reported in the 1990s demonstrated an OS benefit with surgical resection for patients with single brain metastases. In a study of 48 patients, Patchell et $\mathrm{al}^{204}$ demonstrated that surgery followed by WBRT compared with WBRT alone improved OS (40 vs 15 weeks in WBRT arm; $P<.01$ ) and functional dependence ( 38 vs 8 weeks; $P<.005$ ), as well as decreased recurrence $(20 \%$ vs $52 \% ; P<.02)$. Similarly, combined surgery and WBRT led to longer survival and functional independence compared with WBRT alone in another randomized study by Vecht and colleagues $(n=63) .{ }^{205} \mathrm{~A}$ third study of 84 patients found no difference in survival between the two strategies; however, patients with extensive systemic disease and lower performance level were included, which likely resulted in poorer outcomes in the surgical arm. ${ }^{206}$

\section{Stereotactic Radiosurgery}

SRS offers an excellent minimally invasive ablative treatment option for brain metastases. Patients undergoing SRS avoid the risk of surgery-related morbidity, and SRS is generally preferred over surgery for patients with small, asymptomatic lesions that do not require surgery and for patients with lesions that are not surgically accessible. ${ }^{203}$ Late side effects of SRS such as symptomatic edema and RT necrosis are relatively uncommon, but may be observed at higher rates when treating larger lesions. ${ }^{207}$

The role of stereotactic SRS alone for limited brain metastases has been established by multiple phase III randomized trials comparing SRS alone to SRS plus WBRT. ${ }^{208-211}$ Collectively, these studies demonstrate comparable OS and superior cognitive preservation and quality of life with SRS alone compared with SRS plus WBRT. The role of SRS for patients with multiple metastases has also continued to expand. A prospective trial of 1194 patients found no differences in OS or neurologic mortality with SRS for 2 to 4 versus 5 to 10 brain metastases. ${ }^{212}$ A number of analyses have suggested that total volume of brain metastases and the rate of developing new brain metastases may be more important prognostic factors for OS than the number of discrete brain metastases. ${ }^{213-216}$ Taken together, patients with multiple lesions but a low total volume of disease, as well as those with relatively indolent rates of developing new CNS lesions, can represent suitable candidates for SRS. Additionally, patients with a favorable histology of the primary tumor (such as breast cancer) or controlled primary tumors can often benefit from SRS regardless of the number of brain metastases present. ${ }^{217,218}$ While brain metastases arising from small-cell lung cancer have historically been treated with WBRT, an international retrospective study suggested that SRS may be suitable in some cases. ${ }^{219}$ Some brain metastases of radioresistant primary tumors such as melanoma and renal cell carcinoma have also been shown to achieve good local control with SRS.220 Other predictors of longer survival with SRS include younger age, good PS, and primary tumor control. ${ }^{213,217,218,221}$ However, there are a number of contemporary series supporting SRS in 
patients with a poor prognosis, with poor KPS, or who are older. $222-225$

Maximal marginal doses for SRS use should be based on tumor volume and range from 15 to 24 Gy when treating lesions with a single fraction of SRS. ${ }^{208,212,226}$ Multifraction SRS may be considered for larger tumors, with the most common doses being $27 \mathrm{~Gy}$ in 3 fractions and 30 Gy in 5 fractions. ${ }^{227-229}$ Contouring guidelines have been published elsewhere. ${ }^{230}$ In the recurrence setting, several patient series have demonstrated local control rates greater than $70 \%$ with SRS for patients with good PS and stable disease who have received prior WBRT. ${ }^{231-234}$

Postoperative SRS also represents an important strategy to improve local control after resection of brain metastases. After resection alone, the rates of local recurrence are relatively high, and have been reported in the range of $50 \%$ at 1 to 2 years in prospective trials. Postoperative SRS to the surgical cavity is supported by a randomized phase III trial including 132 patients with resected brain metastases (1-3 lesions). This trial demonstrated that postoperative SRS was associated with a higher 12-month local recurrence-free rate compared with no postoperative treatment $(72 \%$ vs $43 \%$, respectively; HR, 0.46; 95\% CI, 0.24-0.88; $P=.015) .{ }^{201} \mathrm{~A}$ separate randomized phase III trial comparing postoperative SRS with postoperative WBRT demonstrated similar OS and better cognitive preservation with a strategy of postoperative SRS, despite superior CNS control outcomes with WBRT. ${ }^{235}$

\section{Whole-Brain Radiation Therapy}

Historically, WBRT was the mainstay of treatment of metastatic lesions in the brain. Although the role of WBRT has diminished over the last several decades, WBRT continues to play a role in the modern era, primarily in clinical scenarios where SRS and surgery are not feasible or indicated (eg, diffuse brain metastases). The standard dosing for WBRT is 30 Gy in 10 fractions or 37.5 Gy in 15 fractions. For patients with poor prognosis, $20 \mathrm{~Gy}$ in 5 fractions may also be used.

The impact of WBRT in addition to SRS has been evaluated in multiple randomized controlled studies. ${ }^{208-211,236}$ A 2018 Cochrane meta-analysis of randomized controlled trials found that the addition of WBRT to SRS alone was associated with better brain control, no differences in OS, and worse neurocognitive outcomes or quality of life in several trials. ${ }^{237}$ The randomized phase III EORTC 22952 trial failed to show an OS benefit from WBRT following resection or SRS, compared with observation, ${ }^{211}$ even in subgroup analyses including only patients with controlled extracranial disease and a favorable prognostic score. ${ }^{238}$ Overall, for patients treated with SRS for brain metastases, the routine addition of WBRT is not recommended due to increased cognitive and quality-of-life toxicity and the lack of an OS benefit.

The randomized phase III noninferiority QUARTZ trial compared WBRT to optimal supportive care in patients with nonsmall cell lung cancer (NSCLC) who were not candidates for SRS, due to various factors including age, PS, and extent of disease. No differences in OS or quality of life were observed with WBRT versus optimal supportive care, which suggests that this population may derive minimal benefit from WBRT. ${ }^{239}$ Moreover, as noted above, a number of studies support SRS for older patients and those with poor prognosis who have historically received WBRT. ${ }^{222-225,240}$ The optimal treatment strategy of brain metastases for patients with a poor prognosis is highly individualized and may call for best supportive care, WBRT, SRS, or trials of CNS-active systemic agents depending on the clinical scenarios.

In light of the well-characterized deleterious cognitive effects of WBRT, ${ }^{209,210,235}$ a number of trials have evaluated strategies to promote cognitive preservation in patients with brain metastases including investigation of neuroprotective agents, anatomic avoidance strategies, and deferral of WBRT in favor of alternate strategies such as SRS or trials of CNS-active systemic agents. In patients undergoing WBRT for brain metastases, the RTOG 0614 $(\mathrm{n}=554)$ compared concurrent and adjuvant memantine, an $N$-methyl-D-aspartate receptor antagonist, to placebo. Memantine was well-tolerated in patients receiving WBRT for brain metastases, and the rates of toxicity were similar to patients receiving placebo. ${ }^{241}$ There was possibly less decline in episodic memory (HVLT-R Delayed Recall) in the memantine arm compared with placebo at 24 weeks $(P=.059)$. The memantine arm had significantly longer time to cognitive decline (HR, 0.78; 95\% CI, 0.62-0.99; $P=.01$ ), and the probability of cognitive function failure at 24 weeks was $54 \%$ in the memantine arm and $65 \%$ in the placebo arm. However, for most cognitive endpoints, no significant differences were observed between memantine and placebo, despite numerical trends that generally favored the memantine arm. For patients with a good prognosis, memantine may be considered during WBRT, as well as after treatment of as long as 6 months.

To evaluate an anatomic-avoidance strategy to promote cognitive preservation, the nonrandomized phase II RTOG-0933 trial showed that reduced radiation dose to the hippocampal neural stem-cell compartment was associated with a smaller decline in recall $(P<.001)$, compared with a historical control. ${ }^{242}$ Based on these results, the phase III NRG-CC001 trial evaluated WBRT with memantine with or without hippocampal avoidance. ${ }^{243}$ There were no significant differences in survival outcomes. However, risk of cognitive failure was significantly lower in the hippocampal avoidance arm than in 
the control arm (HR, $0.76 ; 95 \% \mathrm{CI}, 0.60-0.98 ; P=.03)$. For patients without tumor in or around the hippocampus, hippocampal-sparing WBRT may be preferred in select patients (eg, those with good prognosis).

In the postoperative setting, phase 3 trials have evaluated the role of WBRT after surgical resection of brain metastases. Patchell conducted a study that randomized 95 patients with single intracranial metastases to surgery with or without adjuvant WBRT. ${ }^{244}$ Postoperative RT was associated with a dramatic reduction in tumor recurrence (18\% vs $70 \%$; $P<.001$ ) and likelihood of neurologic deaths ( $14 \%$ vs $44 \% ; P=.003$ ). OS, a secondary endpoint, showed no difference between the arms. The aforementioned EORTC 22952 trial randomized patients treated with local therapy (surgery or SRS) to observation versus WBRT. ${ }^{211}$ Patients randomized to WBRT were found to have superior brain disease control and less death form neurologic causes, but inferior QOL and no differences in OS. ${ }^{21,245}$ The NCCTG N107C/CEC-3 randomized phase III trial included 194 patients with resected brain metastases randomized to either postoperative SRS or WBRT. ${ }^{235}$ Although there was no significant difference between the treatment arms for OS, cognitive deterioration at 6 months was less frequent in the SRS arm than in the WBRT arm $(52 \%$ vs $85 \%$, respectively; $P<.001$ ), and cognitive deterioration-free survival was also greater for postoperative SRS compared with WBRT (median 3.7 months vs median 3.0 months; HR, 0.47; 95\% CI, 0.35-0.63; $P<.001)$. In another phase III trial, 215 patients with 1-3 brain metastases from melanoma were randomized to either WBRT or observation following local treatment with surgery or SRS. ${ }^{246}$ Though local failure rate was significantly lower in the WBRT arm $(20.0 \%$ vs $33.6 \%$, respectively; $P=.03)$, there were no significant differences between the study arms for intracranial failure, OS, and deterioration in performance status. Further, grade 1 to 2 toxicity during the first 2-4 months was more frequently reported in the WBRT arm.

\section{Systemic Therapy}

Many tumors that metastasize to the brain are not chemosensitive or have already been heavily pretreated with organ-specific effective agents. Poor penetration through the BBB is an additional concern. ${ }^{198}$ However, there are increasing numbers of systemic treatment options with demonstrated activity in the brain, and it is now reasonable to treat some of these patients (ie, those with asymptomatic brain metastases) with systemic therapy upfront instead of upfront SRS or WBRT. Specific recommended regimens are based on effective treatment of the primary tumor. Studies have demonstrated that some regimens are effective for treatment of brain metastases for certain types of cancer, notably melanoma (eg, dabrafenib/trametinib for BRAF v600E positive disease, ${ }^{247}$ ipilimumab/nivolumab ${ }^{248}$ ), NSCLC (eg, osimertinib for EGFR T790M positive disease, ${ }^{249-251}$ brigatinib, alectinib, and ceritinib for ALK-positive disease, ${ }^{252,253}$ pembrolizumab and nivolumab for PD-L1-positive disease ${ }^{254,255}$ ), and specific combinations for certain breast cancers (capecitabine with lapatinib or neratinib for HER2-positive disease, ${ }^{256-258}$ tucatinib/trastuzumab/capecitabine for previously treated HER2-positive disease ${ }^{259}$ ). There are also an increasing number of "basket" studies that evaluate the efficacy of targeted therapy options for a specific mutation or biomarker, regardless of tumor type. For example, the TRK inhibitors larotrectinib and entrectinib were found to be active in patients with brain metastases from NTRK gene fusion-positive solid tumors. ${ }^{260,261}$

As CNS-active systemic agents are changing paradigms for the management of brain metastases, it is important to acknowledge that there is a paucity of prospective data to characterize optimal strategies regarding radiation and systemic therapy combinations or sequencing. When considering a trial of upfront systemic therapy alone for brain metastases, a multidisciplinary discussion between medical and radiation oncology is recommended. Ongoing CNS surveillance with brain MRIs is essential to allow early interventions in cases of progression or inadequate response.

\section{NCCN Recommendations}

\section{Workup}

Patients who present with a single mass or multiple lesions on MRI or CT imaging suggestive of metastatic cancer to the brain, and who do not have a known primary, require a careful systemic workup with chest $\mathrm{X}$-ray or CT with contrast, abdominal or pelvic CT with contrast, or other tests as indicated. Whole-body PET/CT may be considered. If no other readily accessible tumor is available for biopsy, a stereotactic or open biopsy resection is indicated to establish a diagnosis. Workup recommendations can be found on LTD-1 for limited brain metastases (see page 1546) and on MU-1 for extensive brain metastases (see page 1550).

\section{Treatment of Limited Metastatic Lesions}

The panel defines "limited" brain metastases as patients for whom SRS represents an effective alternative to WBRT, but with more cognitive protection. ${ }^{212}$ Because brain metastases are often managed by physicians from multiple disciplines, the NCCN Panel encourages multidisciplinary consultation prior to treatment of optimal planning. Treatment recommendations for limited brain metastases can be found on LTD-2 (page 1547).

Surgical resection may be considered in select cases (eg, for management of mass effect or other symptoms; 
for tumors $>3 \mathrm{~cm}$ that are surgically accessible; if there is no other readily accessible tumor to be biopsied). For patients with newly diagnosed or stable systemic disease, treatment options include SRS (preferred) and WBRT. When patients are managed with SRS, NCCN does not recommend the routine addition of WBRT, as this approach has been consistently associated with cognitive deterioration and no difference in survival. ${ }^{209}$ The management of patients with disseminated systemic disease or poor prognosis should be individualized and may include strategies of best supportive care, WBRT, SRS, or a trial of CNS-active systemic agents; multidisciplinary evaluation is encouraged.

In patients with systemic cancers with options for CNS-active systemic therapies, (eg, ALK or EGFR mutations in NSCLC; BRAF mutations in metastatic melanoma), upfront systemic therapy alone may be considered in carefully selected, asymptomatic patients. When considering a trial of upfront systemic therapy alone for brain metastases, NCCN recommends a multidisciplinary discussion between medical and radiation oncologists and ongoing CNS surveillance with brain MRIs to allow for early interventions in cases of progression or inadequate response.

Patients should be followed with brain MRI every 2 to 3 months for 1-2 years and then every 4 to 6 months indefinitely. Closer follow-up every 2 months may be particularly helpful for patients treated with SRS or systemic therapy alone. ${ }^{210}$ Evaluation of potential disease recurrence can be confounded by treatment effects of SRS. Tumor sampling may be indicated to discern recurrence versus treatment effect in some cases. Upon detection of recurrent disease, prior therapy clearly influences the choice of further therapies. Patients with recurrent CNS disease should be assessed for local versus systemic disease, because therapy will differ. For local recurrences, patients who were previously treated with surgery only can receive the following options: 1) surgery with consideration of SRS or RT to the surgical bed, 2) single-dose or fractionated SRS, 3) WBRT, or 4) systemic therapy. However, patients who previously received WBRT generally should not undergo WBRT at recurrence due to concern regarding neurotoxicity. If the patient had previous SRS with a durable response for $>6$ months, reconsider SRS if imaging or biopsy supports active tumor and not necrosis. Repeat SRS to a prior location is a category $2 \mathrm{~B}$ recommendation.

If isolated CNS disease progression occurs in the setting of limited systemic treatment options and poor PS, management of brain metastases should be individualized and may include best supportive care, WBRT, SRS, and CNS-active systemic agents. WBRT reirradiation is generally discouraged due to toxicity to cognition and quality of life and should be administered only in highly selected circumstances. Full treatment recommendations can be found on LTD-3 for recurrent disease (see page 1548) and on LTD-4 for relapsed disease (see page 1549).

\section{Treatment of Extensive Metastatic Lesions}

Patients diagnosed with extensive metastatic lesions should generally be treated with WBRT or SRS as primary therapy. For WBRT dosing, the standard regimens are $30 \mathrm{~Gy}$ in 10 fractions or $37.5 \mathrm{~Gy}$ in 15 fractions. For patients with poor neurologic performance, a more rapid course of RT can be considered (20 Gy, delivered in 5 fractions). SRS may be considered in select patients, particularly those with good PS and low overall tumor volume. Some patients may be eligible for upfront systemic therapy treatment. Palliative neurosurgery may also be considered if a lesion is causing a life-threatening mass effect, hemorrhage, or hydrocephalus. Full treatment recommendations for extensive brain metastases can be found on MU-1 (page 1550).

After WBRT or SRS, patients should have a repeat contrast-enhanced MRI scan every 2 to 3 months for 1-2 years, then every 4 to 6 months indefinitely. Treatment of recurrences are individualized and may include best supportive care, surgery, WBRT, SRS, or a trial of CNS-active systemic therapy; multidisciplinary review is recommended. Repeat WBRT is generally discouraged due to toxicity to cognition and quality of life and should only be administered in highly selected circumstances. Treatment recommendations for recurrent disease can be found on MU-2 (page 1551).

\section{References}

1. Louis DN, Perry A, Reifenberger G, et al. The 2016 World Health Organization Classification of Tumors of the Central Nervous System: a summary. Acta Neuropathol 2016;131: 803-820.

2. Yan $\mathrm{H}$, Parsons DW, Jin G, et al. IDH1 and IDH2 mutations in gliomas. N Engl J Med 2009;360:765-773

3. Houillier $\mathrm{C}$, Wang $\mathrm{X}$, Kaloshi $\mathrm{G}$, et al. IDH1 or IDH2 mutations predict longer survival and response to temozolomide in low-grade gliomas. Neurology 2010;75:1560-1566

4. Cancer Genome Atlas Research Network. Brat DJ, Verhaak RG, Aldape $K D$, et al. Comprehensive, integrative genomic analysis of diffuse lowergrade gliomas. N Engl J Med 2015;372:2481-2498. Available at: http:// www.ncbi.nlm.nih.gov/pubmed/26061751.

5. Eckel-Passow JE, Lachance DH, Molinaro AM, et al. Glioma groups based on $1 p / 19 q, I D H$, and TERT promoter mutations in tumors. N Engl $\mathrm{J}$ Med 2015;372:2499-2508.

6. Wiestler B, Capper D, Sill M, et al. Integrated DNA methylation and copy-number profiling identify three clinically and biologically 
relevant groups of anaplastic glioma. Acta Neuropathol 2014;128: 561-571.

7. Weller M, Weber RG, Willscher E, et al. Molecular classification of diffuse cerebral WHO grade II/III gliomas using genome- and transcriptomewide profiling improves stratification of prognostically distinct patient groups. Acta Neuropathol 2015;129:679-693.

8. Nitta M, Muragaki Y, Maruyama T, et al. Proposed therapeutic strategy for adult low-grade glioma based on aggressive tumor resection. Neurosurg Focus 2015;38:E7.

9. Baumert BG, Hegi ME, van den Bent MJ, et al. Temozolomide chemotherapy versus radiotherapy in high-risk low-grade glioma (EORTC 22033-26033): a randomised, open-label, phase 3 intergroup study. Lancet Oncol 2016;17:1521-1532.

10. Sanson M, Marie $Y$, Paris S, et al. Isocitrate dehydrogenase 1 codon 132 mutation is an important prognostic biomarker in gliomas. J Clin Oncol 2009;27:4150-4154.

11. Sahm F, Reuss D, Koelsche C, et al. Farewell to oligoastrocytoma: in situ molecular genetics favor classification as either oligodendroglioma or astrocytoma. Acta Neuropathol 2014;128:551-559.

12. Pai T, Epari S, Desai $S$, et al. Histological spectrum of oligodendroglial tumors: Only a subset shows $1 p / 19 q$ codeletion. Neurol India 2017;65: 113-120.

13. Neumann JE, Dorostkar MM, Korshunov A, et al. Distinct histomorphology in molecular subgroups of glioblastomas in young patients. J Neuropathol Exp Neurol 2016;75:408-414.

14. Dubbink HJ, Atmodimedjo PN, Kros JM, et al. Molecular classification of anaplastic oligodendroglioma using next-generation sequencing: a report of the prospective randomized EORTC Brain Tumor Group 26951 phase III trial. Neuro-oncol 2016;18:388-400.

15. Labussière $M$, Idbaih $A$, Wang $X W$, et al. All the $1 \mathrm{p} 19 \mathrm{q}$ codeleted gliomas are mutated on IDH1 or IDH2. Neurology 2010;74:1886-1890.

16. Horbinski C. What do we know about IDH1/2 mutations so far, and how do we use it? Acta Neuropathol 2013;125:621-636.

17. Jiao Y, Killela PJ, Reitman ZJ, et al. Frequent ATRX, CIC, FUBP1 and IDH1 mutations refine the classification of malignant gliomas. Oncotarget 2012;3:709-722.

18. Leeper HE, Caron AA, Decker PA, et al. IDH mutation, 1p19q codeletion and ATRX loss in WHO grade II gliomas. Oncotarget 2015;6: 30295-30305

19. Reuss DE, Sahm F, Schrimpf D, et al. ATRX and IDH1-R132H immunohistochemistry with subsequent copy number analysis and IDH sequencing as a basis for an "integrated" diagnostic approach for adult astrocytoma, oligodendroglioma and glioblastoma. Acta Neuropathol 2015;129:133-146

20. Arita $\mathrm{H}$, Narita Y, Fukushima S, et al. Upregulating mutations in the TERT promoter commonly occur in adult malignant gliomas and are strongly associated with total 1p19q loss. Acta Neuropathol 2013;126:267-276.

21. Killela PJ, Reitman ZJ, Jiao Y, et al. TERT promoter mutations occur frequently in gliomas and a subset of tumors derived from cells with low rates of self-renewal. Proc Natl Acad Sci USA 2013;110:6021-6026.

22. Kristensen BW, Priesterbach-Ackley LP, Petersen JK, et al. Molecular pathology of tumors of the central nervous system. Ann Oncol 2019;30: 1265-1278.

23. Meyronet D, Esteban-Mader M, Bonnet C, et al. Characteristics of H3 K27M-mutant gliomas in adults. Neuro-oncol 2017;19:1127-1134.

24. Brat DJ, Aldape K, Colman H, et al. cIMPACT-NOW update 5: recommended grading criteria and terminologies for IDH-mutant astrocytomas. Acta Neuropathol 2020;139:603-608.

25. Jiang H, Cui Y, Wang J, et al. Impact of epidemiological characteristics of supratentorial gliomas in adults brought about by the 2016 world health organization classification of tumors of the central nervous system. Oncotarget 2017;8:20354-20361.

26. Wick W, Roth P, Hartmann C, et al. Long-term analysis of the NOA-04 randomized phase III trial of sequential radiochemotherapy of anaplastic glioma with PCV or temozolomide. Neuro-oncol 2016;18:1529-1537.

27. Cairncross JG, Wang M, Jenkins RB, et al. Benefit from procarbazine, lomustine, and vincristine in oligodendroglial tumors is associated with mutation of IDH. J Clin Oncol 2014;32:783-790.

28. Everhard S, Kaloshi G, Crinière E, et al. MGMT methylation: a marker of response to temozolomide in low-grade gliomas. Ann Neurol 2006;60: 740-743.

29. Gorovets D, Kannan K, Shen R, et al. IDH mutation and neuroglial developmental features define clinically distinct subclasses of lower grade diffuse astrocytic glioma. Clin Cancer Res 2012;18: 2490-2501.

30. Wahl M, Phillips JJ, Molinaro AM, et al. Chemotherapy for adult lowgrade gliomas: clinical outcomes by molecular subtype in a phase II study of adjuvant temozolomide. Neuro-oncol 2017;19:242-251.

31. Arita H, Yamasaki $K$, Matsushita Y, et al. A combination of TERT promoter mutation and MGMT methylation status predicts clinically relevant subgroups of newly diagnosed glioblastomas. Acta Neuropathol Commun 2016;4:79

32. Pekmezci M, Rice T, Molinaro AM, et al. Adult infiltrating gliomas with WHO 2016 integrated diagnosis: additional prognostic roles of ATRX and TERT. Acta Neuropathol 2017;133:1001-1016.

33. Möllemann M, Wolter M, Felsberg J, et al. Frequent promoter hypermethylation and low expression of the MGMT gene in oligodendroglial tumors. Int J Cancer 2005;113:379-385.

34. Hegi ME, Diserens AC, Gorlia T, et al. MGMT gene silencing and benefit from temozolomide in glioblastoma. N Engl J Med 2005;352: 997-1003.

35. Hegi ME, Diserens AC, Godard S, et al. Clinical trial substantiates the predictive value of O-6-methylguanine-DNA methyltransferase promoter methylation in glioblastoma patients treated with temozolomide. Clin Cancer Res 2004;10:1871-1874.

36. Bell EH, Zhang P, Fisher BJ, et al. Association of MGMT promoter methylation status with survival outcomes in patients with high-risk glioma treated with radiotherapy and temozolomide: an analysis from the NRG Oncology/RTOG 0424 trial. JAMA Oncol 2018;4: 1405-1409.

37. Malmström A, Grønberg BH, Marosi C, et al. Temozolomide versus standard 6-week radiotherapy versus hypofractionated radiotherapy in patients older than 60 years with glioblastoma: the Nordic randomised, phase 3 trial. Lancet Oncol 2012;13:916-926.

38. Wick W, Platten M, Meisner C, et al. Temozolomide chemotherapy alone versus radiotherapy alone for malignant astrocytoma in the elderly: the NOA-08 randomised, phase 3 trial. Lancet Oncol 2012;13:707-715.

39. Khuong-Quang DA, Buczkowicz P, Rakopoulos P, et al. K27M mutation in histone H3.3 defines clinically and biologically distinct subgroups of pediatric diffuse intrinsic pontine gliomas. Acta Neuropathol 2012;124: 439-447

40. Sturm D, Witt $H$, Hovestadt $V$, et al. Hotspot mutations in H3F3A and IDH1 define distinct epigenetic and biological subgroups of glioblastoma. Cancer Cell 2012;22:425-437.

41. Korshunov A, Capper D, Reuss D, et al. Histologically distinct neuroepithelial tumors with histone $3 \mathrm{G} 34$ mutation are molecularly similar and comprise a single nosologic entity. Acta Neuropathol 2016;131: 137-146.

42. Horbinski C. To BRAF or not to BRAF: is that even a question anymore? J Neuropathol Exp Neurol 2013;72:2-7.

43. Hawkins C, Walker E, Mohamed N, et al. BRAF-KIAA1549 fusion predicts better clinical outcome in pediatric low-grade astrocytoma. Clin Cancer Res 2011;17:4790-4798.

44. Horbinski C, Nikiforova MN, Hagenkord JM, et al. Interplay among BRAF, p16, p53, and MIB1 in pediatric low-grade gliomas. Neuro-oncol 2012;14:777-789.

45. Penman CL, Faulkner C, Lowis SP, et al. Current understanding of BRAF alterations in diagnosis, prognosis, and therapeutic targeting in pediatric low-grade gliomas. Front Oncol 2015;5:54.

46. Kleinschmidt-DeMasters BK, Aisner DL, Birks DK, et al. Epithelioid GBMs show a high percentage of BRAF V600E mutation. Am J Surg Pathol 2013;37:685-698.

47. Mistry M, Zhukova N, Merico D, et al. BRAF mutation and CDKN2A deletion define a clinically distinct subgroup of childhood secondary high-grade glioma. J Clin Oncol 2015;33:1015-1022.

48. Chapman PB, Hauschild A, Robert C, et al.BRIM-3 Study Group. Improved survival with vemurafenib in melanoma with BRAF V600E mutation. N Engl J Med 2011;364:2507-2516.

49. McArthur GA, Chapman PB, Robert C, et al. Safety and efficacy of vemurafenib in BRAF(V600E) and BRAF(V600K) mutation-positive melanoma (BRIM-3): extended follow-up of a phase 3, randomised, openlabel study. Lancet Oncol 2014;15:323-332.

50. Kaley $T$, Touat M, Subbiah V, et al. BRAF inhibition in BRAF(V600)-mutant gliomas: results from the VE-BASKET Study. J Clin Oncol 2018;36: 3477-3484.

51. Chang S, Zhang P, Cairncross JG, et al. Phase III randomized study of radiation and temozolomide versus radiation and nitrosourea therapy for 
anaplastic astrocytoma: results of NRG Oncology RTOG 9813. Neurooncol 2017;19:252-258.

52. Olar A, Wani KM, Alfaro-Munoz KD, et al. IDH mutation status and role of WHO grade and mitotic index in overall survival in grade II-III diffuse gliomas. Acta Neuropathol 2015;129:585-596.

53. Hegi ME, Genbrugge E, Gorlia T, et al. MGMT promoter methylation cutoff with safety margin for selecting glioblastoma patients into trials omitting temozolomide: a pooled analysis of four clinical trials. Clin Cancer Res 2019;25:1809-1816.

54. Ostrom QT, Gittleman $\mathrm{H}, \mathrm{Xu}$ J, et al. CBTRUS statistical report: primary brain and other central nervous system tumors diagnosed in the United States in 2009-2013. Neuro-oncol 2016;18(suppl_5):v1-v75.

55. Chang EF, Potts MB, Keles GE, et al. Seizure characteristics and control following resection in 332 patients with low-grade gliomas. J Neurosurg 2008;108:227-235.

56. Schiff $D$, Van den Bent $M$, Vogelbaum MA, et al. Recent developments and future directions in adult lower-grade gliomas: Society for NeuroOncology (SNO) and European Association of Neuro-Oncology (EANO) consensus. Neuro-oncol 2019;21:837-853.

57. Piepmeier J, Christopher S, Spencer D, et al. Variations in the natural history and survival of patients with supratentorial low-grade astrocytomas. Neurosurgery 1996;38:872-879.

58. Afra D, Osztie E, Sipos $L$, et al. Preoperative history and postoperative survival of supratentorial low-grade astrocytomas. Br J Neurosurg 1999; 13:299-305.

59. Gallo P, Cecchi PC, Locatelli F, et al. Pleomorphic xanthoastrocytoma: long-term results of surgical treatment and analysis of prognostic factors. Br J Neurosurg 2013;27:759-764.

60. Giannini C, Scheithauer BW, Burger PC, et al. Pleomorphic xanthoastrocytoma: what do we really know about it? Cancer 1999;85:2033-2045.

61. Ida CM, Rodriguez FJ, Burger PC, et al. Pleomorphic xanthoastrocytoma: natural history and long-term follow-up. Brain Pathol 2015;25:575-586.

62. Varshneya K, Sarmiento JM, Nuño $M$, et al. A national perspective of adult gangliogliomas. J Clin Neurosci 2016;30:65-70.

63. Tahiri Elousrouti L, Lamchahab M, Bougtoub N, et al. Subependymal giant cell astrocytoma (SEGA): a case report and review of the literature. J Med Case Reports 2016;10:35.

64. Roth J, Roach ES, Bartels U, et al. Subependymal giant cell astrocytoma: diagnosis, screening, and treatment. Recommendations from the International Tuberous Sclerosis Complex Consensus Conference 2012 Pediatr Neurol 2013;49:439-444.

65. Adriaensen ME, Schaefer-Prokop CM, Stijnen T, et al. Prevalence of subependymal giant cell tumors in patients with tuberous sclerosis and a review of the literature. Eur J Neurol 2009;16:691-696.

66. Goh S, Butler W, Thiele EA. Subependymal giant cell tumors in tuberous sclerosis complex. Neurology 2004;63:1457-1461.

67. Skalicky AM, Rentz AM, Liu Z, et al. The burden of subependymal giant cell astrocytomas associated with tuberous sclerosis complex: results of a patient and caregiver survey. J Child Neurol 2015;30:563-569.

68. Franz DN, Bissler JJ, McCormack FX. Tuberous sclerosis complex: neurological, renal and pulmonary manifestations. Neuropediatrics 2010;41:199-208

69. Sun P, Kohrman M, Liu J, et al. Outcomes of resecting subependymal giant cell astrocytoma (SEGA) among patients with SEGA-related tuberous sclerosis complex: a national claims database analysis. Curr Med Res Opin 2012;28:657-663.

70. Berhouma M, Dubourg J, Messerer M. Neurology: Letter to the editor. Re: Sun P, Kohrman M, Liu J et al. Outcomes of resecting subependymal giant cell astrocytoma (SEGA) among patients with SEGA-related tuberous sclerosis complex: a national claims database analysis. Curr Med Res Opin 2012;28:657-63. Curr Med Res Opin 2012;28:1571-1572., author reply 1572-1573.

71. Wen P, Stein A, van den Bent M, et al. ACTR-30. Updated efficacy and safety of dabrafenib plus trametinib in patients with recurrent/refractory BRAF V600E-mutated high-grade glioma (HGG) and low-grade glioma (LGG). Neuro-oncol 2019;21(Supplement_6):vi19-vi20.

72. Brown NF, Carter $T$, Kitchen N, et al. Dabrafenib and trametinib in BRAFV600E mutated glioma. CNS Oncol 2017;6:291-296.

73. Marks AM, Bindra RS, DiLuna ML, et al. Response to the BRAF/MEK inhibitors dabrafenib/trametinib in an adolescent with a BRAF V600E mutated anaplastic ganglioglioma intolerant to vemurafenib. Pediatr Blood Cancer 2018;65:e26969.

74. Franz DN, Belousova E, Sparagana S, et al. Efficacy and safety of everolimus for subependymal giant cell astrocytomas associated with tuberous sclerosis complex (EXIST-1): a multicentre, randomised, placebo-controlled phase 3 trial. Lancet 2013;381:125-132.

75. Franz DN, Belousova E, Sparagana S, et al. Everolimus for subependymal giant cell astrocytoma in patients with tuberous sclerosis complex: 2-year open-label extension of the randomised EXIST-1 study. Lancet Oncol 2014:15:1513-1520.

76. Wahl M, Chang SM, Phillips JJ, et al. Probing the phosphatidylinositol 3-kinase/mammalian target of rapamycin pathway in gliomas: A phase 2 study of everolimus for recurrent adult low-grade gliomas. Cancer 2017; 123:4631-4639.

77. Ebrahimi-Fakhari D, Franz DN. Pharmacological treatment strategies for subependymal giant cell astrocytoma (SEGA). Expert Opin Pharmacother 2020;21:1329-1336.

78. Ostrom QT, Cioffi G, Gittleman H, et al. CBTRUS Statistical Report: Primary Brain and Other Central Nervous System Tumors Diagnosed in the United States in 2012-2016. Neuro-oncol 2019;21(Suppl 5):v1-v100.

79. Pignatti F, van den Bent $M$, Curran $D$, et al. Prognostic factors for survival in adult patients with cerebral low-grade glioma. J Clin Oncol 2002;20: 2076-2084.

80. Daniels TB, Brown PD, Felten SJ, et al. Validation of EORTC prognostic factors for adults with low-grade glioma: a report using intergroup 86-72 51. Int J Radiat Oncol Biol Phys 2011;81:218-224.

81. Lo SS, Cho KH, Hall WA, et al. Does the extent of surgery have an impact on the survival of patients who receive postoperative radiation therapy for supratentorial low-grade gliomas? Int J Cancer 2001; 96(S1, Suppl) 71-78.

82. Shaw $E$, Arusell $R$, Scheithauer $B$, et al. Prospective randomized trial of low-versus high-dose radiation therapy in adults with supratentorial lowgrade glioma: initial report of a North Central Cancer Treatment Group/ Radiation Therapy Oncology Group/Eastern Cooperative Oncology Group study. J Clin Oncol 2002;20:2267-2276.

83. Lo SS, Hall WA, Cho KH, et al. Radiation dose response for supratentorial low-grade glioma--institutional experience and literature review. J Neurol Sci 2003;214:43-48.

84. Shaw EG, Berkey B, Coons SW, et al. Recurrence following neurosurgeon-determined gross-total resection of adult supratentorial low-grade glioma: results of a prospective clinical trial. J Neurosurg 2008;109:835-841.

85. Jackson RJ, Fuller GN, Abi-Said D, et al. Limitations of stereotactic biopsy in the initial management of gliomas. Neuro-oncol 2001;3: 193-200.

86. Villena Martín M, Pena Pardo FJ, Jiménez Aragón F, et al. Metabolic targeting can improve the efficiency of brain tumor biopsies. Semin Oncol 2020;47:148-154

87. Brown TJ, Bota DA, van Den Bent MJ, et al. Management of low-grade glioma: a systematic review and meta-analysis. Neurooncol Pract 2019;6: 249-258.

88. Yang K, Nath S, Koziarz A, et al. Biopsy versus subtotal versus gross tota resection in patients with low-grade glioma: a systematic review and meta-analysis. World Neurosurg 2018;120:e762-e775.

89. Tang S, Liao J, Long Y. Comparative assessment of the efficacy of gross total versus subtotal total resection in patients with glioma: a metaanalysis. Int J Surg 2019;63:90-97.

90. McGirt MJ, Chaichana KL, Attenello FJ, et al. Extent of surgical resection is independently associated with survival in patients with hemispheric infiltrating low-grade gliomas. Neurosurgery 2008;63:700-707., author reply 707-708.

91. Jakola AS, Myrmel KS, Kloster R, et al. Comparison of a strategy favoring early surgical resection vs a strategy favoring watchful waiting in lowgrade gliomas. JAMA 2012;308:1881-1888.

92. Berger MS, Deliganis AV, Dobbins J, et al. The effect of extent of resection on recurrence in patients with low grade cerebral hemisphere gliomas. Cancer 1994;74:1784-1791.

93. Kiliç T, Ozduman K, Elmaci I, et al. Effect of surgery on tumor progression and malignant degeneration in hemispheric diffuse low-grade astrocytomas. J Clin Neurosci 2002;9:549-552.

94. Roelz R, Strohmaier D, Jabbarli R, et al. Residual tumor volume as best outcome predictor in low grade glioma - a nine-years near-randomized survey of surgery vs biopsy. Sci Rep 2016;6:32286.

95. Karim AB, Maat B, Hatlevoll R, et al. A randomized trial on dose-response in radiation therapy of low-grade cerebral glioma: European Organization for Research and Treatment of Cancer (EORTC) Study 22844. Int J Radiat Oncol Biol Phys 1996;36:549-556.

96. van den Bent MJ, Afra D, de Witte O, et al. Long-term efficacy of early versus delayed radiotherapy for low-grade astrocytoma and 
oligodendroglioma in adults: the EORTC 22845 randomised trial. Lancet 2005;366:985-990.

97. Gorlia T, Wu W, Wang M, et al. New validated prognostic models and prognostic calculators in patients with low-grade gliomas diagnosed by central pathology review: a pooled analysis of EORTC/RTOG/ NCCTG phase III clinical trials. Neuro-oncol 2013;15:1568-1579.

98. Shaw EG, Wang M, Coons SW, et al. Randomized trial of radiation therapy plus procarbazine, lomustine, and vincristine chemotherapy for supratentorial adult low-grade glioma: initial results of RTOG 9802. J Clin Oncol 2012;30:3065-3070.

99. Buckner JC, Shaw EG, Pugh SL, et al. Radiation plus procarbazine, CCNU, and vincristine in low-grade glioma. N Engl J Med 2016;374: 1344-1355.

100. Bell EH, Zhang P, Shaw EG, et al. Comprehensive genomic analysis in NRG Oncology/RTOG 9802: a phase III trial of radiation versus radiation plus procarbazine, lomustine (CCNU), and vincristine in high-risk lowgrade glioma. J Clin Oncol 2020;JCO1902983:JCO1902983.

101. Fisher BJ, Hu C, Macdonald DR, et al. Phase 2 study of temozolomidebased chemoradiation therapy for high-risk low-grade gliomas: preliminary results of Radiation Therapy Oncology Group 0424. Int J Radiat Oncol Biol Phys 2015;91:497-504.

102. Navarria P, Pessina F, Cozzi L, et al. Can advanced new radiation therapy technologies improve outcome of high grade glioma (HGG) patients? analysis of 3D-conformal radiotherapy (3DCRT) versus volumetric-modulated arc therapy (VMAT) in patients treated with surgery, concomitant and adjuvant chemo-radiotherapy. BMC Cancer 2016;16:362

103. Ding M, Newman F, Chen $C$, et al. Dosimetric comparison between 3DCRT and IMRT using different multileaf collimators in the treatment of brain tumors. Med Dosim 2009;34:1-8.

104. Shaw EG, Daumas-Duport C, Scheithauer BW, et al. Radiation therapy in the management of low-grade supratentorial astrocytomas. J Neurosurg 1989;70:853-861.

105. Indelicato DJ, Rotondo RL, Uezono H, et al. Outcomes following proton therapy for pediatric low-grade glioma. Int J Radiat Oncol Biol Phys 2019;104:149-156.

106. Nahed BV, Redjal N, Brat DJ, et al. Management of patients with recurrence of diffuse low grade glioma: A systematic review and evidence-based clinical practice guideline. J Neurooncol 2015;125: 609-630.

107. Ramakrishna R, Hebb A, Barber J, et al. Outcomes in reoperated lowgrade gliomas. Neurosurgery 2015;77:175-184., discussion 184

108. Smith JS, Chang EF, Lamborn KR, et al. Role of extent of resection in the long-term outcome of low-grade hemispheric gliomas. J Clin Oncol 2008;26:1338-1345

109. Uppstrom TJ, Singh R, Hadjigeorgiou GF, et al. Repeat surgery for recurrent low-grade gliomas should be standard of care. Clin Neurol Neurosurg 2016;151:18-23.

110. Kesari S, Schiff D, Drappatz J, et al. Phase II study of protracted daily temozolomide for low-grade gliomas in adults. Clin Cancer Res 2009;15: 330-337

111. Nicholson HS, Kretschmar CS, Krailo M, et al. Phase 2 study of temozolomide in children and adolescents with recurrent central nervous system tumors: a report from the Children's Oncology Group. Cancer 2007;110:1542-1550.

112. Soffietti R, Rudà R, Bradac GB, et al. PCV chemotherapy for recurrent oligodendrogliomas and oligoastrocytomas. Neurosurgery 1998;43: 1066-1073.

113. Moghrabi A, Friedman HS, Ashley DM, et al. Phase II study of carboplatin (CBDCA) in progressive low-grade gliomas. Neurosurg Focus 1998;4:e3.

114. Brandes AA, Basso U, Vastola F, et al. Carboplatin and teniposide as third-line chemotherapy in patients with recurrent oligodendroglioma or oligoastrocytoma: a phase II study. Ann Oncol 2003;14 1727-1731.

115. Triebels VH, Taphoorn MJ, Brandes AA, et al. Salvage PCV chemotherapy for temozolomide-resistant oligodendrogliomas. Neurology 2004;63:904-906.

116. van den Bent MJ, Smits M, Kros JM, et al. Diffuse infiltrating oligodendroglioma and astrocytoma. J Clin Oncol 2017;35: 2394-2401.

117. van den Bent MJ, Baumert B, Erridge SC, et al. Interim results from the CATNON trial (EORTC study 26053-22054) of treatment with concurrent and adjuvant temozolomide for $1 \mathrm{p} / 19 \mathrm{q}$ non-co-deleted anaplastic glioma: a phase 3, randomised, open-label intergroup study. Lancet 2017;390:1645-1653

118. Perry JR, Laperriere N, O'Callaghan CJ, et al. Short-course radiation plus temozolomide in elderly patients with glioblastoma. N Engl J Med 2017 376:1027-1037.

119. Stupp R, Mason WP, van den Bent MJ, et al. Radiotherapy plus concomitant and adjuvant temozolomide for glioblastoma. N Engl J Med 2005;352:987-996.

120. Acharya S, Robinson CG, Michalski JM, et al. Association of $1 \mathrm{p} / 19 \mathrm{q}$ codeletion and radiation necrosis in adult cranial gliomas after proton or photon therapy. Int J Radiat Oncol Biol Phys 2018;101: 334-343.

121. Wen PY, Weller M, Lee EQ, et al. Glioblastoma in adults: a Society for Neuro-Oncology (SNO) and European Society of Neuro-Oncology (EANO) consensus review on current management and future directions. Neuro Oncol 2020. Accessed August 26, 2020. Available at: https:// www.ncbi.nlm.nih.gov/pubmed/32328653

122. Arevalo OD, Soto $C$, Rabiei $P$, et al. Assessment of glioblastoma response in the era of bevacizumab: longstanding and emergent challenges in the imaging evaluation of pseudoresponse. Front Neuro 2019:10:460.

123. Li XZ, Li YB, Cao Y, et al. Prognostic implications of resection extent for patients with glioblastoma multiforme: a meta-analysis. J Neurosurg Sci 2017;61:631-639.

124. Beiko J, Suki D, Hess KR, et al. IDH1 mutant malignant astrocytomas are more amenable to surgical resection and have a survival benefit associated with maximal surgical resection. Neuro-oncol 2014;16:81-91.

125. Molinaro AM, Hervey-Jumper S, Morshed RA, et al. Association of maximal extent of resection of contrast-enhanced and non-contrastenhanced tumor with survival within molecular subgroups of patients with newly diagnosed glioblastoma. JAMA Oncol 2020;6:495-503.

126. Barker FG II, Chang SM, Gutin PH, et al. Survival and functional status after resection of recurrent glioblastoma multiforme. Neurosurgery 1998;42:709-720., discussion 720-723.

127. Park JK, Hodges T, Arko L, et al. Scale to predict survival after surgery for recurrent glioblastoma multiforme. J Clin Oncol 2010;28:3838-3843.

128. Kristiansen $\mathrm{K}$, Hagen S, Kollevold T, et al. Combined modality therapy of operated astrocytomas grade III and IV. Confirmation of the value of postoperative irradiation and lack of potentiation of bleomycin on survival time: a prospective multicenter trial of the Scandinavian Glioblastoma Study Group. Cancer 1981;47:649-652.

129. Walker MD, Alexander E, Jr., Hunt WE, et al. Evaluation of BCNU and/o radiotherapy in the treatment of anaplastic gliomas. A cooperative clinical trial. J Neurosurg 1978;49:333-343.

130. Cabrera AR, Kirkpatrick JP, Fiveash JB, et al. Radiation therapy for glioblastoma: executive summary of an American Society for Radiation Oncology Evidence-Based Clinical Practice Guideline. Pract Radiat Oncol 2016:6:217-225.

131. Roa W, Brasher PM, Bauman G, et al. Abbreviated course of radiation therapy in older patients with glioblastoma multiforme: a prospective randomized clinical trial. J Clin Oncol 2004:22:1583-1588.

132. Roa W, Kepka L, Kumar N, et al. International Atomic Energy Agency randomized phase III study of radiation therapy in elderly and/or frail patients with newly diagnosed glioblastoma multiforme. J Clin Oncol 2015;33:4145-4150.

133. Finazzi T. One week of radiotherapy for glioblastoma: a noninferiority trial? J Clin Oncol 2016;34:2192.

134. Kim H, Leiby BE, Shi W. Too little, too soon: short-course radiotherapy in elderly patients with glioblastoma. J Clin Oncol 2016;34:2191-2192.

135. Stupp R, Hegi ME, Mason WP, et al.. Effects of radiotherapy with concomitant and adjuvant temozolomide versus radiotherapy alone on survival in glioblastoma in a randomised phase III study: 5-yea analysis of the EORTC-NCIC trial. Lancet Oncol 2009;10:459-466.

136. van den Bent MJ, Carpentier AF, Brandes AA, et al. Adjuvant procarbazine, lomustine, and vincristine improves progression-free surviva but not overall survival in newly diagnosed anaplastic oligodendrogliomas and oligoastrocytomas: a randomized European Organisation for Research and Treatment of Cancer phase III trial. J Clin Oncol 2006;24:2715-2722

137. van den Bent MJ, Brandes AA, Taphoorn MJ, et al. Adjuvant procarbazine, lomustine, and vincristine chemotherapy in newly diagnosed anaplastic oligodendroglioma: long-term follow-up of EORTC brain tumor group study 26951. J Clin Oncol 2013;31:344-350.

138. Cairncross G, Berkey B, Shaw E, et al. Phase III trial of chemotherapy plus radiotherapy compared with radiotherapy alone for pure and mixed 
anaplastic oligodendroglioma: Intergroup Radiation Therapy Oncology Group Trial 9402. J Clin Oncol 2006;24:2707-2714.

139. Cairncross G, Wang M, Shaw E, et al. Phase III trial of chemoradiotherapy for anaplastic oligodendroglioma: long-term results of RTOG 9402. J Clin Oncol 2013;31:337-343.

140. Jaeckle KA, Ballman KV, van den Bent M, et al. CODEL: phase III study of $\mathrm{RT}, \mathrm{RT}+$ temozolomide (TMZ), or TMZ for newly-diagnosed $1 \mathrm{p} / 19 \mathrm{q}$ codeleted oligodendroglioma. Analysis from the initial study design. Neuro Oncol Accessed August 26, 2020. Available at: https://www.ncbi. nlm.nih.gov/pubmed/32678879

141. van den Bent MJ, Erridge SC, Vogelbaum MA, et al. Second interim and first molecular analysis of the EORTC randomized phase III intergroup CATNON trial on concurrent and adjuvant temozolomide in anaplastic glioma without 1p/19q codeletion. ASCO 2019. Accessed August 26 , 2020. Available at: https://meetinglibrary.asco.org/record/173361/ abstract

142. Clarke JL, Iwamoto FM, Sul J, et al. Randomized phase II trial of chemoradiotherapy followed by either dose-dense or metronomic temozolomide for newly diagnosed glioblastoma. J Clin Oncol 2009;27: 3861-3867.

143. Gilbert MR, Wang M, Aldape KD, et al. Dose-dense temozolomide for newly diagnosed glioblastoma: a randomized phase III clinical trial. J Clin Oncol 2013;31:4085-4091.

144. Stupp R, Hegi ME, Gorlia T, et al. Cilengitide combined with standard treatment for patients with newly diagnosed glioblastoma with methylated MGMT promoter (CENTRIC EORTC 26071-22072 study): a multicentre, randomised, open-label, phase 3 trial. Lancet Oncol 2014; 15:1100-1108

145. Nabors $L B$, Fink $K L$, Mikkelsen $T$, et al. Two cilengitide regimens in combination with standard treatment for patients with newly diagnosed glioblastoma and unmethylated MGMT gene promoter: results of the open-label, controlled, randomized phase II CORE study. Neuro-oncol 2015;17:708-717.

146. Blumenthal DT, Gorlia T, Gilbert MR, et al. Is more better? The impact of extended adjuvant temozolomide in newly diagnosed glioblastoma: a secondary analysis of EORTC and NRG Oncology/RTOG. Neuro-oncol 2017;19:1119-1126.

147. Balana C, Vaz MA, Sepulveda JM, et al. A phase II randomized, multicenter, open-label trial of continuing adjuvant temozolomide beyond six cycles in patients with glioblastoma (GEINO 14-01) [published online April 24, 2020]. Neuro Oncol, doi: 10.1093/neuonc/noaa107 https:// www.ncbi.nlm.nih.gov/pubmed/32328662

148. Glas M, Happold C, Rieger J, et al. Long-term survival of patients with glioblastoma treated with radiotherapy and lomustine plus temozolomide. J Clin Oncol 2009;27:1257-1261

149. Herrlinger $U$, Rieger J, Koch $D$, et al. Phase II trial of lomustine plus temozolomide chemotherapy in addition to radiotherapy in newly diagnosed glioblastoma: UKT-03. J Clin Oncol 2006;24:4412-4417.

150. Herrlinger $U$, Tzaridis T, Mack F, et al.. Lomustine-temozolomide combination therapy versus standard temozolomide therapy in patients with newly diagnosed glioblastoma with methylated MGMT promoter (CeTeG/NOA-09): a randomised, open-label, phase 3 trial. Lancet 2019; 393:678-688.

151. Weller J, Tzaridis T, Mack F, et al. Health-related quality of life and neurocognitive functioning with lomustine-temozolomide versus temozolomide in patients with newly diagnosed, MGMT-methylated glioblastoma (CeTeG/NOA-09): a randomised, multicentre, open-label, phase 3 trial. Lancet Oncol 2019;20:1444-1453.

152. Stupp R, Taillibert $S$, Kanner AA, et al. Maintenance therapy with tumortreating fields plus temozolomide vs temozolomide alone for glioblastoma: a randomized clinical trial. JAMA 2015;314:2535-2543.

153. Stupp R, Taillibert $\mathrm{S}$, Kanner A, et al. Effect of tumor-treating fields plus maintenance temozolomide vs maintenance temozolomide alone on survival in patients with glioblastoma: a randomized clinical trial. JAMA 2017;318:2306-2316.

154. Taphoorn MJB, Dirven L, Kanner AA, et al. Influence of treatment with tumor-treating fields on health-related quality of life of patients with newly diagnosed glioblastoma: a secondary analysis of a randomized clinical trial. JAMA Oncol 2018:4:495-504.

155. Chamberlain MC. Treatment of patients with newly diagnosed glioblastoma. JAMA 2016;315:2348

156. Sampson JH. Alternating electric fields for the treatment of glioblastoma. JAMA 2015;314:2511-2513.

157. Perry JR, Rizek P, Cashman R, et al. Temozolomide rechallenge in recurrent malignant glioma by using a continuous temozolomide schedule: the "rescue" approach. Cancer 2008:113:2152-2157.
158. Brandes $\mathrm{AA}$, Tosoni $\mathrm{A}$, Amistà $\mathrm{P}$, et al. How effective is $\mathrm{BCNU}$ in recurrent glioblastoma in the modern era? A phase II trial. Neurology 2004;63: 1281-1284.

159. Reithmeier T, Graf E, Piroth T, et al. BCNU for recurrent glioblastoma multiforme: efficacy, toxicity and prognostic factors. BMC Cancer 2010; 10:30.

160. Wick W, Puduvalli VK, Chamberlain MC, et al. Phase III study of enzastaurin compared with lomustine in the treatment of recurrent intracranial glioblastoma. J Clin Oncol 2010;28:1168-1174.

161. Taal W, Oosterkamp HM, Walenkamp AM, et al. Single-agent bevacizumab or lomustine versus a combination of bevacizumab plus lomustine in patients with recurrent glioblastoma (BELOB trial): a randomised controlled phase 2 trial. Lancet Oncol 2014;15:943-953.

162. Friedman HS, Prados MD, Wen PY, et al. Bevacizumab alone and in combination with irinotecan in recurrent glioblastoma. J Clin Oncol 2009:27:4733-4740.

163. Wick W, Gorlia T, Bendszus M, et al. Lomustine and bevacizumab in progressive glioblastoma. N Engl J Med 2017;377:1954-1963.

164. Ameratunga $\mathrm{M}$, Pavlakis $\mathrm{N}$, Wheeler $\mathrm{H}$, et al. Anti-angiogenic therapy for high-grade glioma. Cochrane Database Syst Rev 2018;11:CD008218.

165. Wick W, Weller $M$, van den Bent $M$, et al. Bevacizumab and recurrent malignant gliomas: a European perspective. J Clin Oncol 2010;28: e188-e189., author reply e190-e192.

166. Kaley T, Nolan C, Carver A, et al. Bevacizumab for acute neurologic deterioration in patients with glioblastoma. CNS Oncol 2013;2:413-418.

167. Lombardi G, De Salvo GL, Brandes AA, et al. Regorafenib compared with lomustine in patients with relapsed glioblastoma (REGOMA): a multicentre, open-label, randomised, controlled, phase 2 trial. Lancet Oncol 2019;20:110-119.

168. Brem H, Piantadosi S, Burger PC, et al. Placebo-controlled trial of safety and efficacy of intraoperative controlled delivery by biodegradable polymers of chemotherapy for recurrent gliomas. Lancet 1995;345: 1008-1012.

169. McGirt MJ, Brem H. Carmustine wafers (Gliadel) plus concomitant temozolomide therapy after resection of malignant astrocytoma: growing evidence for safety and efficacy. Ann Surg Oncol 2010;17: 1729-1731.

170. Stupp R, Wong ET, Kanner AA, et al. NovoTTF-100A versus physician's choice chemotherapy in recurrent glioblastoma: a randomised phase III trial of a novel treatment modality. Eur J Cancer 2012;48:2192-2202.

171. Kazmi F, Soon YY, Leong YH, et al. Re-irradiation for recurrent glioblastoma (GBM): a systematic review and meta-analysis. J Neurooncol 2019:142:79-90

172. Mohammadi AM, Sullivan TB, Barnett GH, et al. Use of high-field intraoperative magnetic resonance imaging to enhance the extent of resection of enhancing and nonenhancing gliomas. Neurosurgery 2014; 74:339-348.

173. Coburger J, Wirtz CR. Fluorescence guided surgery by 5-ALA and intraoperative MRI in high grade glioma: a systematic review. J Neurooncol 2019;141:533-546.

174. Panageas KS, Iwamoto FM, Cloughesy TF, et al. Initial treatment patterns over time for anaplastic oligodendroglial tumors. Neuro-oncol 2012;14 761-767

175. Brandes AA, Nicolardi L, Tosoni A, et al. Survival following adjuvant PCV or temozolomide for anaplastic astrocytoma. Neuro-oncol 2006;8: 253-260.

176. Levin VA, Silver $P$, Hannigan J, et al. Superiority of post-radiotherapy adjuvant chemotherapy with CCNU, procarbazine, and vincristine (PCV) over BCNU for anaplastic gliomas: NCOG 6G61 final report. Int J Radiat Oncol Biol Phys 1990;18:321-324.

177. Minniti G, Scaringi C, Lanzetta G, et al. Standard (60 Gy) or short-course (40 Gy) irradiation plus concomitant and adjuvant temozolomide for elderly patients with glioblastoma: a propensity-matched analysis. Int $J$ Radiat Oncol Biol Phys 2015;91:109-115.

178. Brandes AA, Franceschi E, Tosoni A, et al. MGMT promoter methylation status can predict the incidence and outcome of pseudoprogression after concomitant radiochemotherapy in newly diagnosed glioblastoma patients. J Clin Oncol 2008;26:2192-2197.

179. Ellingson BM, Chung C, Pope WB, et al. Pseudoprogression, radionecrosis, inflammation or true tumor progression? challenges associated with glioblastoma response assessment in an evolving therapeutic landscape. J Neurooncol 2017;134:495-504.

180. Tsien C, Galbán CJ, Chenevert TL, et al. Parametric response map as an imaging biomarker to distinguish progression from pseudoprogression in high-grade glioma. J Clin Oncol 2010;28:2293-2299. 
181. Fink J, Born D, Chamberlain MC. Pseudoprogression: relevance with respect to treatment of high-grade gliomas. Curr Treat Options Oncol 2011;12:240-252

182. Perry JR, Bélanger K, Mason WP, et al. Phase II trial of continuous doseintense temozolomide in recurrent malignant glioma: RESCUE study. J Clin Oncol 2010;28:2051-2057.

183. Weller M, Tabatabai G, Kästner B, et al. MGMT promoter methylation is a strong prognostic biomarker for benefit from dose-intensified temozolomide rechallenge in progressive glioblastoma: the DIRECTOR trial. Clin Cancer Res 2015;21:2057-2064.

184. Yung WK, Albright RE, Olson J, et al. A phase II study of temozolomide vs. procarbazine in patients with glioblastoma multiforme at first relapse. Br J Cancer 2000;83:588-593.

185. Prados M, Rodriguez L, Chamberlain M, et al. Treatment of recurrent gliomas with 1,3-bis(2-chloroethyl)-1-nitrosourea and alphadifluoromethylornithine. Neurosurgery 1989;24:806-809.

186. Chamberlain MC, Johnston S. Bevacizumab for recurrent alkylatorrefractory anaplastic oligodendroglioma. Cancer 2009;115:1734-1743.

187. Chamberlain MC, Johnston S. Salvage chemotherapy with bevacizumab for recurrent alkylator-refractory anaplastic astrocytoma. J Neurooncol 2009;91:359-367.

188. Norden AD, Young GS, Setayesh K, et al. Bevacizumab for recurrent malignant gliomas: efficacy, toxicity, and patterns of recurrence. Neurology 2008;70:779-787.

189. Cloughesy T, Prados MD, Mikkelsen T. A phase 2 randomized noncomparative clinical trial of the effect of bevacizumab alone or in combination with irinotecan on 6-month progression free survival in recurrent refractory glioblastoma. [abstract] J Clin Oncol 2008;26(Suppl 15):2010b

190. Kreisl TN, Kim L, Moore K, et al. Phase II trial of single-agent bevacizumab followed by bevacizumab plus irinotecan at tumor progression in recurrent glioblastoma. J Clin Oncol 2009;27:740-745.

191. Vredenburgh JJ, Desjardins A, Herndon JE II, et al. Phase II trial of bevacizumab and irinotecan in recurrent malignant glioma. Clin Cancer Res 2007;13:1253-1259.

192. Carvalho BF, Fernandes AC, Almeida DS, et al. Second-line chemotherapy in recurrent glioblastoma: a 2-cohort study. Oncol Res Treat 2015;38:348-354.

193. Schmidt F, Fischer J, Herrlinger U, et al. PCV chemotherapy for recurrent glioblastoma. Neurology 2006;66:587-589.

194. Barnholtz-Sloan JS, Sloan AE, Davis FG, et al. Incidence proportions of brain metastases in patients diagnosed (1973 to 2001) in the Metropolitan Detroit Cancer Surveillance System. J Clin Oncol 2004;22: 2865-2872.

195. Schouten LJ, Rutten J, Huveneers HA, et al. Incidence of brain metastases in a cohort of patients with carcinoma of the breast, colon, kidney, and lung and melanoma. Cancer 2002;94:2698-2705.

196. Fox BD, Cheung VJ, Patel AJ, et al. Epidemiology of metastatic brain tumors. Neurosurg Clin N Am 2011;22:1-6.

197. Gould J. Breaking down the epidemiology of brain cancer. Nature 2018; 561:S40-S41.

198. Maher EA, Mietz J, Arteaga CL, et al. Brain metastasis: opportunities in basic and translational research. Cancer Res 2009;69:6015-6020.

199. Lin NU, Bellon JR, Winer EP. CNS metastases in breast cancer. J Clin Oncol 2004;22:3608-3617.

200. Eichler AF, Loeffler JS. Multidisciplinary management of brain metastases. Oncologist 2007;12:884-898.

201. Mahajan A, Ahmed S, McAleer MF, et al. Post-operative stereotactic radiosurgery versus observation for completely resected brain metastases: a single-centre, randomised, controlled, phase 3 trial. Lancet Oncol 2017;18:1040-1048.

202. Churilla TM, Chowdhury IH, Handorf E, et al. Comparison of local control of brain metastases with stereotactic radiosurgery vs surgical resection: a secondary analysis of a randomized clinical trial. JAMA Oncol 2019;5: 243-247. Available at: https://www.ncbi.nlm.nih.gov/pubmed/ 30419088 .

203. Ewend MG, Morris DE, Carey LA, et al. Guidelines for the initial management of metastatic brain tumors: role of surgery, radiosurgery, and radiation therapy. J Natl Compr Canc Netw 2008;6:505-513., quiz 514.

204. Patchell RA, Tibbs PA, Walsh JW, et al. A randomized trial of surgery in the treatment of single metastases to the brain. N Engl J Med 1990;322: 494-500.

205. Vecht CJ, Haaxma-Reiche H, Noordijk EM, et al. Treatment of single brain metastasis: radiotherapy alone or combined with neurosurgery? Ann Neurol 1993;33:583-590.
206. Mintz AH, Kestle J, Rathbone MP, et al. A randomized trial to assess the efficacy of surgery in addition to radiotherapy in patients with a single cerebral metastasis. Cancer 1996:78:1470-1476.

207. Suh JH. Stereotactic radiosurgery for the management of brain metastases. N Engl J Med 2010;362:1119-1127.

208. Aoyama $\mathrm{H}$, Shirato $H$, Tago $M$, et al. Stereotactic radiosurgery plus whole-brain radiation therapy vs stereotactic radiosurgery alone for treatment of brain metastases: a randomized controlled trial. JAMA 2006;295:2483-2491.

209. Brown PD, Jaeckle K, Ballman KV, et al. Effect of radiosurgery alone vs radiosurgery with whole brain radiation therapy on cognitive function in patients with 1 to 3 brain metastases: a randomized clinical trial. JAMA 2016;316:401-409.

210. Chang EL, Wefel JS, Hess KR, et al. Neurocognition in patients with brain metastases treated with radiosurgery or radiosurgery plus whole-brain irradiation: a randomised controlled trial. Lancet Oncol 2009:10: 1037-1044.

211. Kocher M, Soffietti $R$, Abacioglu $U$, et al. Adjuvant whole-brain radiotherapy versus observation after radiosurgery or surgical resection of one to three cerebral metastases: results of the EORTC 22952-26001 study. J Clin Oncol 2011;29:134-141.

212. Yamamoto $M$, Serizawa $T$, Shuto $T$, et al. Stereotactic radiosurgery for patients with multiple brain metastases (JLGK0901): a multi-institutional prospective observational study. Lancet Oncol 2014;15:387-395.

213. Bhatnagar AK, Flickinger JC, Kondziolka D, et al. Stereotactic radiosurgery for four or more intracranial metastases. Int J Radiat Oncol Biol Phys 2006;64:898-903.

214. Banfill KE, Bownes PJ, St Clair SE, et al. Stereotactic radiosurgery for the treatment of brain metastases: impact of cerebral disease burden on survival. Br J Neurosurg 2012;26:674-678.

215. Chang WS, Kim HY, Chang JW, et al. Analysis of radiosurgical results in patients with brain metastases according to the number of brain lesions: is stereotactic radiosurgery effective for multiple brain metastases? J Neurosurg 2010; 113(Special_Supplement, Suppl)73-78.

216. Farris M, McTyre ER, Cramer CK, et al. Brain metastasis velocity: a novel prognostic metric predictive of overall survival and freedom from wholebrain radiation therapy after distant brain failure following upfront radiosurgery alone. Int J Radiat Oncol Biol Phys 2017;98:131-141.

217. Karlsson B, Hanssens $P$, Wolff $R$, et al. Thirty years' experience with gamma knife surgery for metastases to the brain. J Neurosurg 2009;111: 449-457.

218. Kased N, Binder DK, McDermott MW, et al. Gamma knife radiosurgery for brain metastases from primary breast cancer. Int J Radiat Oncol Biol Phys 2009;75:1132-1140.

219. Rusthoven CG, Yamamoto M, Bernhardt D, et al. Evaluation of first-line radiosurgery vs whole-brain radiotherapy for small cell lung cancer brain metastases: the FIRE-SCLC Cohort Study. JAMA Oncol 2020;6: 1028-1037.

220. Hanson PW, Elaimy AL, Lamoreaux WT, et al. A concise review of the efficacy of stereotactic radiosurgery in the management of melanoma and renal cell carcinoma brain metastases. World J Surg Oncol 2012;10: 176.

221. Hunter GK, Suh JH, Reuther AM, et al. Treatment of five or more brain metastases with stereotactic radiosurgery. Int J Radiat Oncol Biol Phys 2012;83:1394-1398.

222. Minniti G, Esposito V, Clarke E, et al. Stereotactic radiosurgery in elderly patients with brain metastases. J Neurooncol 2013;111:319-325.

223. Higuchi $Y$, Yamamoto $M$, Serizawa $T$, et al. Stereotactic radiosurgery in elderly patients with brain metastases: comparison with non-elderly patients using database of a multi-institutional prospective observational study (JLGK0901-Elderly). J Neurooncol 2019;144:393-402.

224. Raman S, Mou B, Hsu F, et al. Whole brain radiotherapy versus stereotactic radiosurgery in poor-prognosis patients with one to 10 brain metastases: a randomised feasibility study. Clin Oncol (R Coll Radiol) 2020;32:442-451.

225. Chen L, Shen C, Redmond KJ, et al. Use of stereotactic radiosurgery in elderly and very elderly patients with brain metastases to limit toxicity associated with whole brain radiation therapy. Int J Radiat Oncol Biol Phys 2017;98:939-947.

226. Shaw $E$, Scott $C$, Souhami $L$, et al. Single dose radiosurgical treatment of recurrent previously irradiated primary brain tumors and brain metastases: final report of RTOG protocol 90-05. Int J Radiat Oncol Biol Phys 2000;47:291-298.

227. Minniti G, Scaringi $C$, Paolini S, et al. Single-fraction versus multifraction $(3 \times 9$ Gy) stereotactic radiosurgery for large $(>2 \mathrm{~cm})$ brain metastases: a 
comparative analysis of local control and risk of radiation-induced brain necrosis. Int J Radiat Oncol Biol Phys 2016;95:1142-1148.

228. Al-Omair A, Soliman H, Xu W, et al. Hypofractionated stereotactic radiotherapy in five daily fractions for post-operative surgical cavities in brain metastases patients with and without prior whole brain radiation. Technol Cancer Res Treat 2013;12:493-499.

229. Soltys SG, Seiger K, Modlin LA, et al. A phase I/II dose-escalation trial of 3-fraction stereotactic radiosurgery (SRS) for large resection cavities of brain metastases. Int J Radiat Oncol Biol Phys 2015;93:S38

230. Soliman $H$, Ruschin $M$, Angelov L, et al. Consensus contouring guidelines for postoperative completely resected cavity stereotactic radiosurgery for brain metastases. Int J Radiat Oncol Biol Phys 2018;100: 436-442.

231. Akyurek S, Chang EL, Mahajan A, et al. Stereotactic radiosurgical treatment of cerebral metastases arising from breast cancer. Am J Clin Oncol 2007;30:310-314.

232. Noël G, Proudhom MA, Valery CA, et al. Radiosurgery for re-irradiation of brain metastasis: results in 54 patients. Radiother Oncol 2001;60: $61-67$

233. Sheehan J, Kondziolka D, Flickinger J, et al. Radiosurgery for patients with recurrent small cell lung carcinoma metastatic to the brain: outcomes and prognostic factors. J Neurosurg 2005; 102(Special_Supplement, Suppl)247-254.

234. Caballero JA, Sneed PK, Lamborn KR, et al. Prognostic factors for survival in patients treated with stereotactic radiosurgery for recurrent brain metastases after prior whole brain radiotherapy. Int J Radiat Oncol Biol Phys 2012;83:303-309.

235. Brown PD, Ballman KV, Cerhan JH, et al. Postoperative stereotactic radiosurgery compared with whole brain radiotherapy for resected metastatic brain disease (NCCTG N107C/CEC.3): a multicentre, randomised, controlled, phase 3 trial. Lancet Oncol 2017;18:1049-1060.

236. Andrews DW, Scott CB, Sperduto PW, et al. Whole brain radiation therapy with or without stereotactic radiosurgery boost for patients with one to three brain metastases: phase III results of the RTOG 9508 randomised trial. Lancet 2004;363:1665-1672.

237. Tsao MN, Xu W, Wong RK, et al. Whole brain radiotherapy for the treatment of newly diagnosed multiple brain metastases. Cochrane Database Syst Rev 2018;1:CD003869.

238. Churilla TM, Handorf E, Collette $S$, et al. Whole brain radiotherapy after stereotactic radiosurgery or surgical resection among patients with one to three brain metastases and favorable prognoses: a secondary analysis of EORTC 22952-26001. Ann Oncol 2017;28:2588-2594.

239. Mulvenna P, Nankivell M, Barton R, et al. Dexamethasone and supportive care with or without whole brain radiotherapy in treating patients with non-small cell lung cancer with brain metastases unsuitable for resection or stereotactic radiotherapy (QUARTZ): results from a phase 3, noninferiority, randomised trial. Lancet 2016;388:2004-2014.

240. Yomo S, Hayashi M. Is upfront stereotactic radiosurgery a rational treatment option for very elderly patients with brain metastases? A retrospective analysis of 106 consecutive patients age 80 years and older. BMC Cancer 2016;16:948.

241. Brown PD, Pugh S, Laack NN, et al. Memantine for the prevention of cognitive dysfunction in patients receiving whole-brain radiotherapy: a randomized, double-blind, placebo-controlled trial. Neuro-oncol 2013; 15:1429-1437.

242. Gondi V, Pugh SL, Tome WA, et al. Preservation of memory with conformal avoidance of the hippocampal neural stem-cell compartment during whole-brain radiotherapy for brain metastases (RTOG 0933): a phase II multi-institutional trial. J Clin Oncol 2014;32:3810-3816.

243. Brown PD, Gondi V, Pugh S, et al.for NRG Oncology. Hippocampal avoidance during whole-brain radiotherapy plus memantine for patients with brain metastases: phase III trial NRG Oncology CC001. J Clin Oncol 2020;38:1019-1029.
244. Patchell RA, Tibbs PA, Regine WF, et al. Postoperative radiotherapy in the treatment of single metastases to the brain: a randomized trial. JAMA 1998;280:1485-1489.

245. Soffietti R, Kocher M, Abacioglu UM, et al. A European Organisation for Research and Treatment of Cancer phase III trial of adjuvant whole-brain radiotherapy versus observation in patients with one to three brain metastases from solid tumors after surgical resection or radiosurgery: quality-of-life results. J Clin Oncol 2013;31:65-72.

246. Hong AM, Fogarty GB, Dolven-Jacobsen K, et al. Adjuvant whole-brain radiation therapy compared with observation after local treatment of melanoma brain metastases: a multicenter, randomized phase III trial. J Clin Oncol 2019;37:3132-3141.

247. Davies MA, Saiag P, Robert $C$, et al. Dabrafenib plus trametinib in patients with BRAFV600-mutant melanoma brain metastases (COMBI-MB): a multicentre, multicohort, open-label, phase 2 trial. Lancet Oncol 2017; 18:863-873.

248. Tawbi HA, Forsyth PA, Algazi A, et al. Combined nivolumab and ipilimumab in melanoma metastatic to the brain. N Engl J Med 2018;379: 722-730.

249. Soria JC, Ohe Y, Vansteenkiste J, et al. Osimertinib in untreated EGFRmutated advanced non-small-cell lung cancer. N Engl J Med 2018;378: 113-125

250. Mok TS, Wu YL, Ahn MJ, et al. Osimertinib or platinum-pemetrexed in EGFR T790M-positive lung cancer. N Engl J Med 2017;376:629-640.

251. Goss G, Tsai CM, Shepherd FA, et al. Osimertinib for pretreated EGFR Thr790Met-positive advanced non-small-cell lung cancer (AURA2): a multicentre, open-label, single-arm, phase 2 study. Lancet Oncol 2016; 17:1643-1652.

252. Camidge DR, Kim HR, Ahn MJ, et al. Brigatinib versus crizotinib in ALKpositive non-small-cell lung cancer. N Engl J Med 2018;379:2027-2039.

253. Gandhi L, Ou SI, Shaw AT, et al. Efficacy of alectinib in central nervous system metastases in crizotinib-resistant ALK-positive non-small-cell lung cancer: comparison of RECIST 1.1 and RANO-HGG criteria. Eur J Cancer 2017;82:27-33

254. Goldberg SB, Gettinger SN, Mahajan A, et al. Pembrolizumab for patients with melanoma or non-small-cell lung cancer and untreated brain metastases: early analysis of a non-randomised, open-label, phase 2 trial. Lancet Oncol 2016;17:976-983.

255. Goldman JW, Crino L, Vokes EE, et al. P2.36: Nivolumab (nivo) in patients (pts) with advanced (adv) NSCLC and central nervous system (CNS) metastases (mets). J Thorac Oncol 2016;11:S238-S239.

256. Saura $C$, Oliveira M, Feng $Y$, et al. Neratinib + capecitabine versus lapatinib + capecitabine in patients with HER2 + metastatic breast cancer previously treated with $\geq 2$ HER2-directed regimens: findings from the multinational, randomized, phase III NALA trial. J Clin Oncol 2019;37(15_suppl):1002-1002.

257. Petrelli $F$, Ghidini $M$, Lonati $V$, et al. The efficacy of lapatinib and capecitabine in HER-2 positive breast cancer with brain metastases: A systematic review and pooled analysis. Eur J Cancer 2017;84:141-148.

258. Freedman RA, Gelman RS, Anders CK, et al. TBCRC 022: a phase II trial of neratinib and capecitabine for patients with human epidermal growth factor receptor 2-positive breast cancer and brain metastases. J Clin Oncol 2019:37:1081-1089.

259. Murthy RK, Loi S, Okines A, et al. Tucatinib, trastuzumab, and capecitabine for HER2-positive metastatic breast cancer. N Engl J Med 2020 382:597-609.

260. Doebele RC, Drilon A, Paz-Ares L, et al. Entrectinib in patients with advanced or metastatic NTRK fusion-positive solid tumours: integrated analysis of three phase 1-2 trials. Lancet Oncol 2020;21:271-282.

261. Hong DS, DuBois SG, Kummar S, et al. Larotrectinib in patients with TRK fusion-positive solid tumours: a pooled analysis of three phase 1/2 clinical trials. Lancet Oncol 2020;21:531-540. 


\section{Individual Disclosures for the NCCN Central Nervous System Cancers Panel}

\begin{tabular}{|c|c|c|c|c|}
\hline Panel Member & $\begin{array}{l}\text { Clinical Research Support/Data Safety } \\
\text { Monitoring Board }\end{array}$ & $\begin{array}{l}\text { Scientific Advisory Boards, Consultant, } \\
\text { or Expert Witness }\end{array}$ & $\begin{array}{l}\text { Promotional Advisory Boards, } \\
\text { Consultant, or Speakers Bureau }\end{array}$ & Specialties \\
\hline Manmeet Ahluwalia, MD" & $\begin{array}{l}\text { AbbVie, Inc.; AstraZeneca Pharmaceuticals LP; } \\
\text { Bayer HealthCare; Bristol-Myers Squibb Company; } \\
\text { Forma Therapeutics, Inc.; Incyte Corporation; } \\
\text { Merck \& Co., Inc.; Mimivax; Novocure; } \\
\text { Pharmacyclics, Inc.; and VBI Vaccines, Inc. }\end{array}$ & $\begin{array}{l}\text { Bayer HealthCare; Forma Therapeutics, Inc.; } \\
\text { Novocure; and Tocagen Inc. }\end{array}$ & None & $\begin{array}{l}\text { Hematology/Hematology Oncology; Medical } \\
\text { Oncology; and Neurology/Neuro-oncology }\end{array}$ \\
\hline Joachim Baehring, MD & None & Forma Therapeutics, Inc & None & Neurology/Neuro-oncology \\
\hline Henry Brem, MD & $\begin{array}{l}\text { Bristol-Myers Squibb Company, and Arbor } \\
\text { Pharmaceuticals, LLC }\end{array}$ & $\begin{array}{l}\text { Accelerating Combination Therapies; Asclepix } \\
\text { Therapeutics; B*Cured: Flighting Brain Cancer; } \\
\text { Camden Partners; Galen Robotics; InSightec; } \\
\text { LikeMinds, Inc.; Nurami Medical; Perosphere Inc.; } \\
\text { and StemGen }\end{array}$ & None & $\begin{array}{l}\text { Surgery/Surgical Oncology, and Neurology/ } \\
\text { Neuro-oncology }\end{array}$ \\
\hline Steven Brem, MD & $\begin{array}{l}\text { Advantage; Medicenna Therapeutics Corp; and } \\
\text { Molecular Targeting Technologies }\end{array}$ & Northwest Biotherapeutics, Inc. & None & Surgery/Surgical Oncology \\
\hline Nicholas Butowski, MD & $\begin{array}{l}\text { AbbVie, Inc.; Amgen Inc. Bayer HealthCare; Ipsen; } \\
\text { Jazz Pharmaceuticals Inc.; Karyopharm } \\
\text { Therapeutics, Inc.; Medicenna Therapeutics Corp; } \\
\text { and Novartis Pharmaceuticals Corporation }\end{array}$ & AbbVie, Inc.; Amgen Inc.; and Cordance & None & $\begin{array}{l}\text { Neurology/Neuro-oncology, and Medical } \\
\text { Oncology }\end{array}$ \\
\hline Jian L. Campian, MD, PhD & NeolmmuneTech, Inc. & AbbVie, Inc. & None & $\begin{array}{l}\text { Neurology/Neuro-oncology, and Medical } \\
\text { Oncology }\end{array}$ \\
\hline $\begin{array}{l}\text { Stephen W. Clark, MD, } \\
\text { PhD }\end{array}$ & None & None & None & Neurology/Neuro-oncology \\
\hline Andrew J. Fabiano, MD & None & None & None & Surgery/Surgical Oncology \\
\hline Peter Forsyth, MD & None & $\begin{array}{l}\text { National Cancer Institute; National Cancer Research } \\
\text { Institute (NCRI); National Institutes of Health (NIH); } \\
\text { and Physical Sciences Oncology Network (PSON) }\end{array}$ & $\begin{array}{l}\text { Bayer HealthCare; Boehringer Ingelheim GmbH; } \\
\text { and Bristol-Myers Squibb Company }\end{array}$ & Neurology/Neuro-oncology \\
\hline $\begin{array}{l}\text { Jona Hattangadi-Gluth, } \\
\text { MD }\end{array}$ & Varian Medical Systems, Inc. & None & Varian Medical Systems, Inc. & Radiotherapy/Radiation Oncology \\
\hline $\begin{array}{l}\text { Matthias Holdhoff, MD, } \\
\text { PhD }\end{array}$ & None & None & None & Medical Oncology \\
\hline Craig Horbinski, MD, PhD & None & None & None & Pathology \\
\hline Larry Junck, MD & None & Orbus Pharmaceutical & None & Neurology/Neuro-oncology \\
\hline Thomas Kaley, MD & None & None & None & Neurology/Neuro-oncology \\
\hline Priya Kumthekar, MD & $\begin{array}{l}\text { AbbVie, Inc.; AstraZeneca Pharmaceuticals LP; } \\
\text { DNAtrix, Inc.; Genentech, Inc.; and Orbus } \\
\text { Therapeutics }\end{array}$ & $\begin{array}{l}\text { Janssen Pharmaceutica Products, LP, and Orbus } \\
\text { Therapeutics }\end{array}$ & None & Neurology/Neuro-oncology \\
\hline Jay S. Loeffler, MD & None & None & None & $\begin{array}{l}\text { Radiotherapy/Radiation Oncology, and Neurology/ } \\
\text { Neuro-oncology }\end{array}$ \\
\hline $\begin{array}{l}\text { Maciej M. Mrugala, MD, } \\
\text { PhD, MPH }\end{array}$ & Arbor Pharmaceuticals, LLC & Arbor Pharmaceuticals, LLC, and Veevo & None & $\begin{array}{l}\text { Neurology/Neuro-oncology, and Medical } \\
\text { Oncology }\end{array}$ \\
\hline Louis Burt Nabors, MD & University of Pennsylvania & $\begin{array}{l}\text { BTG International, Inc., and Karyopharm } \\
\text { Therapeutics, Inc. }\end{array}$ & None & Neurology/Neuro-oncology \\
\hline Seema Nagpal, MD & $\begin{array}{l}\text { Agios, Inc.; Berg Health LLC; Inovio } \\
\text { Pharmaceuticals, Inc.; and PharmAbcine }\end{array}$ & None & Novocure & $\begin{array}{l}\text { Medical Oncology, and Neurology/Neuro- } \\
\text { oncology }\end{array}$ \\
\hline Manjari Pandey, MD & None & None & None & Medical Oncology \\
\hline Ian Parney, MD, PhD & None & Oncorus, Inc. & None & $\begin{array}{l}\text { Neurology/Neuro-oncology, and Surgery/Surgical } \\
\text { Oncology }\end{array}$ \\
\hline $\begin{array}{l}\text { Katherine Peters, MD, } \\
\text { PhD }\end{array}$ & $\begin{array}{l}\text { AbbVie, Inc.; Agios, Inc.;Biomimetix } \\
\text { Pharmaceutical, Inc.; and Novocure }\end{array}$ & Agios, Inc., and Bayer HealthCare & None & Neurology/Neuro-oncology \\
\hline Jana Portnow, MD & None & None & None & $\begin{array}{l}\text { Medical Oncology, and Neurology/Neuro- } \\
\text { oncology }\end{array}$ \\
\hline Vinay K. Puduvalli, MD & $\begin{array}{l}\text { AbbVie, Inc.; BeiGene; and Ziopharm Oncology, } \\
\text { Inc. }\end{array}$ & $\begin{array}{l}\text { Nektar Therapeutics; Orbus Therapeutics; and } \\
\text { SK Life Science, Inc. }\end{array}$ & $\begin{array}{l}\text { Nektar Therapeutics; Orbus Therapeutics; and } \\
\text { SK Life Science, Inc. }\end{array}$ & Neurology/Neuro-oncology \\
\hline lan Robins, MD, PhD & None & None & None & Medical Oncology \\
\hline Jason Rockhill, MD, PhD & None & None & None & Radiotherapy/Radiation Oncology \\
\hline Chad Rusthoven, MD & Takeda Pharmaceuticals North America, Inc. & None & None & Radiotherapy/Radiation Oncology \\
\hline Nicole Shonka, MD & None & None & None & $\begin{array}{l}\text { Medical Oncology, and Neurology/Neuro- } \\
\text { oncology }\end{array}$ \\
\hline $\begin{array}{l}\text { Dennis C. Shrieve, MD, } \\
\text { PhD }\end{array}$ & None & None & None & Radiotherapy/Radiation Oncology \\
\hline Lode J. Swinnen, MB, ChBa & None & Karyopharm Therapeutics, Inc. & None & Hematology/Hematology Oncology \\
\hline Stephanie Weiss, MD" & None & None & None & Radiotherapy/Radiation Oncology \\
\hline Patrick Yung Wen, MD & None & $\begin{array}{l}\text { Agios, Inc.;Boston Pharmaceuticals, Inc.;Elevate } \\
\text { Bio; Karyopharm Therapeutics, Inc.; Merck \& Co., } \\
\text { Inc.; Novartis Pharmaceuticals Corporation; QED; } \\
\text { and Tocagen Inc. }\end{array}$ & None & Neurology/Neuro-oncology \\
\hline Nicole E. Willmarth, PhD & None & None & None & Patient Advocacy \\
\hline
\end{tabular}

The NCCN Guidelines Staff have no conflicts to disclose.

aThe following individuals have disclosed that they have an employment/governing board, patent, equity, or royalty: Manmeet Ahluwalia, MD: Cytodyn; Doctible; and Mimivax

Andrew J. Fabiano, MD: Arbor Pharmaceuticals - Clincial Research Support as Part of VIGILANT study

Andrew J. Fabiano, MD: Arbor Pharmaceut
Vinay K. Puduvalli: Gilead Sciences, Inc.

Lode J. Swinnen, MBChB: Johns Hopkins University

Stephanie Weiss, MD: AstraZeneca Pharmaceuticals LP, and Regeneron Pharmaceuticals, Inc. 